\title{
MODELO AUTO-REGRESSIVO PARA ANÁLISE DE EXPERIMENTOS COM VACAS EM LACTAÇÃO
}

\author{
ADALBERTO JOSÉ CROCCI
}

Orientador: Prof. Dr. CÁSSIO ROBERTO DE MELO GODOI

Tese apresentada à Escola Superior de Agricultura "Luiz de Queiroz" da Universidade de São Paulo, para obtenção do Título de Doutor em Agronomia. Área de Concentração: Estatística e Experimentação Agronômica.

PIR A C I C A B A

Estado de São Paulo - Brasil

Janeiro, 1984 
Aos meus pais ROSA e NELSON

DEDICO

A minha esposa ROSANE e aos meus filhos MARTANA, SUZANE e RAFAEL 


\section{AGRADECTMENTOS}

Ao Professor Dr. Cāssio Roberto de Melo Godoi, pela orienta ção eficiente durante o curso e na realização deste trabalho.

A Faculdade de Ciēncias Agrárias e Veterinārias, Campus de Ja boticabal - UNESP, pela oportunidade oferecida.

Aos colegas do Departamento de Ciēncias Exatas da Faculdade de Ciências Agrárias e Veterinárias, Campus de Jaboticabal - UNESP, pelo incentivo e colaboração.

Ao Professor Dr. Euclides Braga Malheiros, pelo auxilio e constante disponibilidade, quer na troca de idéias, quex na parte de com putação.

Ao Professor Dr. Dilermando Perecin, pelas valiosas sugestões e revisão do texto.

A CAPES, em nome da Coordenação de Capacitação de Docentes da da FCAVJ-UNESP, pelo auxílio financeiro prestado.

Aos funcionários da Unidade de Processamento de Dados da FCAVJ-UNESP, pela constante colaboração:

A Maria de Lourdes Moretto, pela dedicação nos trabalhos de datilografia.

A todos aqueles que de uma forma ou de outra contribuiram pa ra a realização deste trabalho. 
INDICE

Pägina

$\operatorname{RESUMO} \ldots \ldots \ldots \ldots \ldots \ldots \ldots \ldots \ldots \ldots \ldots \ldots \ldots \ldots \ldots \ldots \ldots \ldots \ldots$

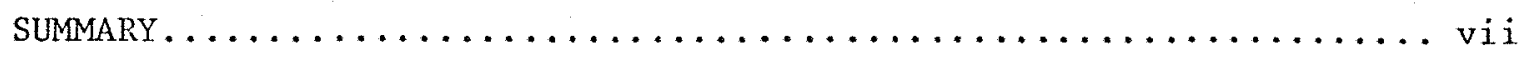

1. Introduç̆ $\ldots \ldots \ldots \ldots \ldots \ldots \ldots \ldots \ldots \ldots \ldots \ldots \ldots \ldots \ldots \ldots \ldots \ldots$

1.1. Apresentação do problema ................... 1

1.2. Limitações do trabalho..................... 4

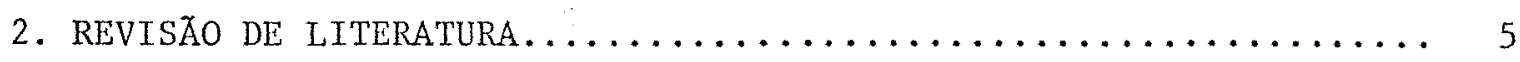

2.1. Delineamentos alternativos ("change-over") ........... 5

2.1.1. Delineamentos de reversão ("switchback")......... 6

2.1.2. Delineamentos rotativos ("rotational").......... 8

2.2. Modelo auto-regressivo em ensaios de lactação.......... 11

3. MATERIAL E METTODOS.......................... 14

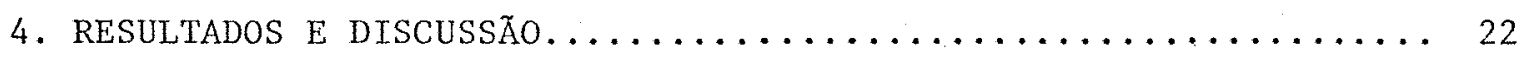

4.1. Anālise da variância no modelo auto-regressivo......... 22

4.1 .1 .0 modelo linear geral .................. 22

4.1.2. Sistema de equações normais............... 25

4.1.3. Soma de quadrados de tratamentos ajustada......... 34

4.1.4. Soma de quadrados de periodos e média ignorando tra tamentos......................... 40

4.1.5. Soma de quadrados total................. 42

4.1.6. Esperança matemática da soma de quadrados de trata mentos ajustada..................... 43

4.1.7. Esperança matemática da soma de quadrados de perío dos e média, ignorando tratamentos............ 46

4.1.8. Esperança matemática da soma de quadrados residual.. 49

4.2. Variāncia de estimativas de efeitos de tratamentos....... 51

4.3. Exemplo ilustrativo da análise da variancia pelo modelo au to-regressivo......................... 53

4.4. Comparação da sensibilidade dos modelos e adequação do mode 10 auto-regressivo........................ 57

5. conclusões................................ 61

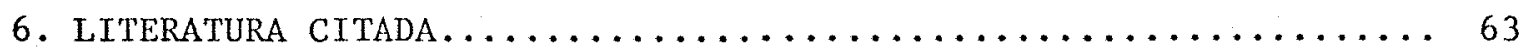

7. TAbElas................................... 69 


\section{MODELO AUTO-REGRESSIVO PARA ANALLISE DE EXPERIMENTOS COM VACAS EM LACTAÇÃO}

Autor: ADALBERTO JOSE CROCCI

Orientador: Prof. Dr. CASSIO ROBERTO DE MELO GODOI

\section{RESUMO}

Os modelos usuais para anälise de dados de produção de le te, como o "switchback" introduzido por LUCAS (1956) ou o delineamento em quadrado latino para os ensaios rotativos, pertencem à classe dos chama dos experimentos em "change-over", nos quais cada unidade experimental re cebe uma seqüencia de värios tratamentos em periodos sucessivos. Tais mo delos apresentam uma estrutura de erros do tipo $\sigma^{2} I$ à custa, em geral, de um nümero excessivo de parâmetros.

Neste trabalho é apresentado um modelo simplificado, deno minado modelo auto-regressivo, no qual os erros apresentam uma distribui ção probabilistica que incorpora a clara correlação existente entre dados de um mesmo animal, com um menor nümero de parāmetros.

Após estabelecido o modelo matemático auto-regressivo pro cede-se à estimação dos parâmetros pelo método dos mínimos quadrados gene ralizados, descrito dentre outros por RAO (1965), e através de ensaios em branco com dados reais de produção de leite, amostrados de uma considera da população finita, simulam-se efeitos aditivos de tratamentos objetivan 
do comparar os modelos, no que se refere à sensibilidade do teste F para a deteção de tais efeitos.

Para este estudo consideram-se três ensaios, escolhidos de forma a se ter uma desejável variação do número de graus de liberdade pa ra o residuo, e coerência com os casos mais comuns encontrados em aplica ções präticas. Cada ensaio é analisado segundo os värios modelos conside rando-se a existéncia ou não de efeitos de tratamentos, bem como diferen tes valores do coeficiente de correlação linear entre periodos de observa ção, para o modelo auto-regressivo.

Os resultados deste trabalho mostram que:

(a) Existe uma boa adequação do modelo auto-regressivo às condições experimentais e que tal adequação piora com o aumento da discre pāncia entre o coeficiente de correlação linear usado e o exato;

(b) o modelo auto-regressivo proposto como competidor do "switchback" ou do rotativo, tem maior sensibilidade para indicar diferen ças entre efeitos de tratamentos, e tal sensibilidade é dependente do coeficiente de correlação linear entre períodos adotado;

(c) A sensibilidade no modelo auto-regressivo é proporcio nal ao número de períodos adotado, indicando assim que tal modelo deve ser preferive1 com.número máximo de períodos possivel. 


\section{AUTOREGRESSIVE MODEL FOR DAIRY COWS EXPERIMENTS}

\section{Author: ADALBERTO JOSE CROCCI}

Orienting: Prof. Dr. CÁSSIO ROBERTO DE MELO GODOI

SUMMARY

The usual models for dairy cows data analysis like the switchback design introduced by LUCAS (1956) or the latin square design for rotational trials, belong to the so-called change-over class of design, in which each experimental unit receaves a sequence of treatments in successi ve periods. Such models present an error structure of $\sigma^{2} I$ type implied, in general, by an excessive number of additive parameters in the model.

A simplified model is presented in this work, namely the autoregressive model, in which the residual deviations present a probabi listic distribution that includes the obvius correlation among data from the same animal, with just a few number of additive parameters.

After the mathematical model has been stablished, parame ter estimation was conducted by the generalized least squares method, des cribed, for instance, by RAO (1965) and, through blank experiments with real dairy data, sampled from a finite fixed population, the "F" statis tics was studied with respect to its statistical properties, specially its sensibility to additive treatment effects. 
For this purpose three designs were considered, selected in a maner to cover a desirable variation of error degrees of freedom and best fitting with actual applied experiments.

Each simulated experiment is analysed under several mathe matical models, considering or not the existence of treatment effects, as well as different values of 1 inear correlation coefficients among periods, only for the autoregressive type.

The main results showed that:

(a) There exists a good fitting of the autoregressive model in the worked experimental conditions and such a fitting is worse when the difference of the real and the used linear correlation coefficient turns bigger;

(b) The proposed autoregressive model as competitor of the switchback and of the rotational models, is more sensible in detecting treatment differences, and such sensibility is dependent of the linear correlation coefficient adopted;

(c) The sensibility of the autoregressive model is propor tional to the number of periods used, showing that such a model is prefe red when the maximum possible number of periods is attained. 
1. INTRODUÇÃO

\subsection{Apresentação do problema}

o presente trabalho aborda experimentos com vacas en lacta ção, nos quais tratamentos são aplicados a unidades experimentais em um nümero de períodos sucessivos, sendo que cada unidade experimental recebe um tratamento diferente em cada período.

Sabe-se que, quando as observações são tomadas seqüencial mente no tempo, como nos ensaios "switchback" ou rotativos, é prática co mum considerar para a análise dos dados a hipótese de independencia dos erros, o que facilita sobremaneira a aplicação de testes estatisticos. En tretanto, para que tal hipötese seja aceitāvel, é necessärio que o modelo matemático inclua todos os parâmetros que possam de alguma forma afetar a estrutura de correlação das observações.

Existem porèm, outros métodos possiveis, certamente menos convenientes em termos de facilidade de construção e aplicação, mas tal vez mais sensiveis para a detecção, por exemplo, de efeitos de tratamen tos. 
Neste trabalho vamos admitir que os erros de observações entre periodos são correlacionados na forma de um processo Markoviano es tacionärio de primeira ordem. Assim se $y_{j}$ representa uma observação no j-ésimo período, então:

$$
y_{j}=a^{\prime} \underline{\beta}+e_{j}
$$

onde $\underset{\sim}{a} \underset{\sim}{\beta}$ é a soma algëbrica dos parâmetros explicativos de $y_{j}$, e

$$
e_{j}=\rho e_{j-1}+\varepsilon_{j}
$$

sendo $\rho$ a correlação linear entre observações de dois periodos consecuti vos, com $|\rho|<1$, e $\varepsilon_{j} \stackrel{i i d}{\sim} N\left(0, \sigma^{2}\right)$ e independentes dos $e_{j}$.

Desta forma para p períodos teremos

$$
\begin{aligned}
& \operatorname{Var}\left\{e_{j}\right\}=\frac{\sigma^{2}}{1-\rho^{2}} \\
& \operatorname{Cov}\left\{e_{j}, e_{j}\right\}=\frac{\sigma^{2}}{1-\rho^{2}} \rho^{\left|j-j^{\prime}\right|} ; j e j^{\prime}=1,2, \ldots, p
\end{aligned}
$$

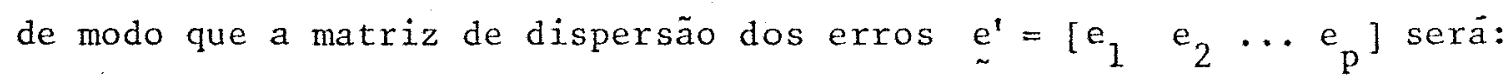

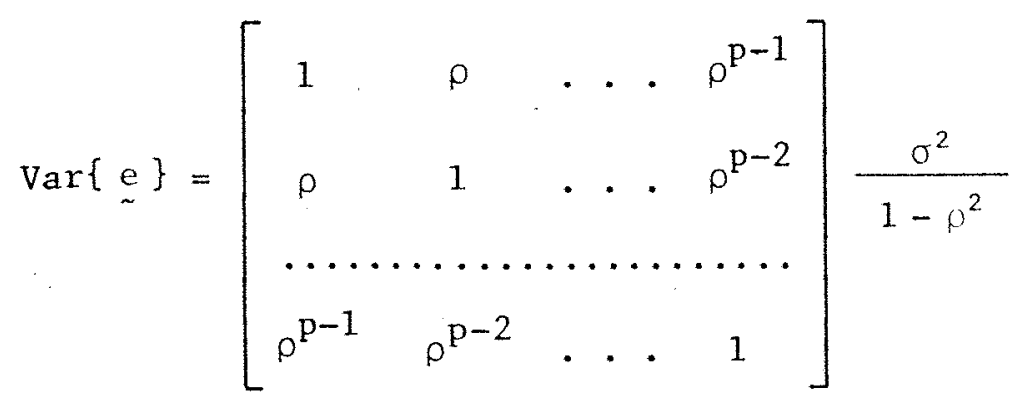

doravante denominada estrutura estocástica auto-regressiva.

Segundo GODOI (1983), para ensaios com vacas em lactação, admitida a estrutura de correlação (1), as observações podem ser descri 
tas pe1o modelo:

$$
y_{i j k_{i j}}=\mu+p_{j}+t_{k_{i j}}+e_{i j k_{i j}}
$$

onde $y_{i j k_{i j}}$ é a observação no $i$-ésimo animal que no $j$-ésimo período re cebeu o tratamento $k$, com:

$$
\begin{aligned}
& \mathbf{i}=1,2, \ldots, \text { a (animais) } \\
& j=1,2, \ldots, p \text { (periodos) } \\
& k=1,2, \ldots, k \text { (tratamentos) com } k_{i j} \neq k_{i j} \text { para } j \neq j^{\prime} .
\end{aligned}
$$

Portanto, para um total de a.p observacões teremos:

$$
{\underset{\sim}{Y}}^{\prime}=\left[\begin{array}{llllll}
y_{11 k_{11}} & y_{12 k_{12}} & \cdots & y_{1 p k_{1 p}} & \cdots & y_{a p k} \\
& & &
\end{array}\right.
$$

cuja matriz de variâncias e covariâncias será:

$$
\operatorname{Var}\{\underline{\sim}\}=V \sigma_{e}^{2}=\left(I_{a} \otimes A\right) \sigma_{e}^{2}
$$

onde $\quad \sigma_{e}^{2}=\frac{\sigma^{2}}{1-\rho^{2}}$

e

$$
\left(I_{a} \otimes A\right) \text { é o produto de Kronecker entre } I_{a} \text { e A dado por }
$$

$$
\left(I_{a} \otimes A\right)=\left[\begin{array}{lllll}
A & \phi & \cdots & . & \phi \\
\phi & A & \cdot & \cdot & \phi \\
\ldots & \ldots & \ldots & \ldots & \ldots \\
\phi & \phi & \cdot & \ddots & A
\end{array}\right]_{a p}
$$

com $\phi$ a matriz nula de ordem $(p \times p) e$ 
$A=\left[\begin{array}{lllll}1 & \rho & \cdot & \rho^{p-1} \\ \rho & 1 & \cdot & \cdot \rho^{p-2} \\ \vdots & \vdots & & \vdots \\ \rho^{p-1} & \rho^{p-2} & \cdot & \cdot\end{array}\right]$

Uma vez adotado o modelo matemático (2) com estrutura de correlações (3), estaremos interessados na sensibilidade do modelo para deteção de diferenças entre efeitos de tratamentos. Para tanto, tal mo delo será comparado com dois outros usuais na análise de dados de produ ção de leite, ou seja, o de delineamento rotativo (quadrados latinos) e o de delineamento de reversão ("switchback").

\subsection{Limitações do trabalho}

Serà considerado neste trabalho, somente a estimação dos efeitos de tratamentos, sem levar em consideração os possiveis efeitos residuais de um tratamento, aplicado em um dado periodo, sobre os perío dos subseqüentes. Na prätica é comum, neste caso, desprezarem-se as pro duções iniciais de cada periodo (uma ou duas semanas), baseando-se a aná 1 ise nas produções restantes.

Por outro lado, os dados da Tabela 1, que serão considera dos na comparação das sensibilidades dos vários modelos citados, podem ser considerados como uma população finita, da qual serão amostrados ani mais e periodos que comporão os vários ensaios. Desta forma podemos afir mar que o coeficiente de correlação linear ( $\rho)$ entre períodos, calculado com tais dados, é o populacional ou exato, o qual será utilizado na meto dologia do trabalho. Portanto, não será abordado o problema da estimação deste coeficiente no ensaio, nem sua influência na sensibilidade dos testes. 


\section{REVISÃO DE LITERATURA}

\subsection{Delineamentos alternativos ("change-over")}

$\mathrm{Na}$ experimentação com animajs, particularmente com vacas em lactação, são de grande utilidade os ensaios alternativos ou "change over", nos quais cada animal recebe durante o experimento uma sequência de dois ou mais tratamentos, de forma que cada animal caracteriza um b1. co. Esses ensaios são em geral classificados em duas categorias, segundo LUCAS (1960):

a) Ensaios de reversão ("switchback")

b) Ensaios rotativos ("rotational"),

os quais se distinguem pela natureza das sequências de tratamentos. Nos rotativos os tratamentos aplicados em uma dada seqüência são todos distin tos, enquanto que nos ensaios de reversão um dado tratamento aparece em uma seqüencia mais de uma vez em periodos não consecutivos.

Esses delineamentos, segundo PATTERSON e LUCAS (1962), são úteis no sentido de permitirem comparações de efeitos de tratamentos com alta precisão, devido à eliminação das diferenças entre seqüencias ou ani 
mais do erro experimental, bem como pela economia de animais para a compa ração de um mesmo nümero de tratamentos.

Os esquemas de ensaio que se seguem ilustram a diferença entre os rotativos e os de reversão, no caso particular de $\mathrm{K}=3$ tratamen tos.

a) Ensaio de Reversão

\begin{tabular}{cccc}
\hline \multirow{2}{*}{ Periodos } & \multicolumn{2}{c}{ Vacas ou sequências } \\
\cline { 2 - 4 } & 1 & 2 & 3 \\
\hline I & $(1)$ & $(2)$ & $(3)$ \\
II & $(2)$ & $(3)$ & $(1)$ \\
III & (1) & (2) & (3)
\end{tabular}

b) Ensaio Rotativo

\begin{tabular}{cccc}
\hline \multirow{2}{*}{ Periodos } & \multicolumn{2}{c}{ Vacas ou sequencias } \\
\cline { 2 - 4 } I & 1 & 2 & 3 \\
\hline II & $(1)$ & $(2)$ & $(3)$ \\
III & $(3)$ & $(1)$ & $(1)$ \\
\hline
\end{tabular}

onde os números entre parênteses codificam os tratamentos.

2.1.1. Delineamentos de reversão ("switchback")

BRANDT (1938) é o primeiro a abordar os ensaios de rever são, considerando o caso de somente dois tratamentos com extensão para três e quatro períodos, apresentando a anälise estatística, a qual é tam bém descrita em SNEDECOR (1946). No artigo, Brandt acrescenta a anālise de covariāncia ajustando as produções de leite para um mesmo teor de gor dura, visando aumentar a sensibilidade do teste $\mathrm{F}$.

Outros autores como TAYLOR e ARMSTRONG (1953) estendem es tes ensaios para mais de dois tratamentos, indicando o método da análise da variancia e introduzindo o conceito de delineamento completo e reduzi do, porém é de LUCAS (1956) a maior contribuição sobre os ensaios de re versão. Neste trabalho o autor aborda a extensão dos delineamentos para 
mais de dois tratamentos, apresentando os esquemas para 3, 4, 5, 6, 7 e 9 tratamentos, cuja construção é baseada nos princípios dos blocos incomple tos balanceados, abordando com detalhes a análise estatistica nos casos comuns e com formação de blocos, além de indicar fórmulas para o cālculo de parcelas perdidas.

Segundo Lucas, a comparação de $\mathrm{K}$ tratamentos exige $\mathrm{K}(\mathrm{K}-1)$ seqüèncias ou animais (delineamento completo) subdivididas em até $\mathrm{K}-1$ blo cos, enquanto que se $\mathrm{K}$ for impar e maior ou igual a 5 , podem ser usados esquemas com $\frac{1}{2} \mathrm{~K}(\mathrm{~K}-1)$ seqüencias de tratamentos (delineamento reduzido) subdivididas em até $\frac{1}{2}(K-1)$ blocos de $K$ seqüências cada.

Por outro lado, visando minimizar o erro experimental, to das as vacas devem estar com a produção entre o pico da lactação e a meta de da gestação seguinte, justificando assim a formação dos citados blocos no caso do nümero de animais disponiveis ser limitado.

GODOI (1972) e GODOI e NOGUEIRA (1972) apresentam a anā1 se da variância do delineamento de Lucas, deduzida a partir do modelo ma temätico

$$
E\left(y_{i j k}\right)=\mu+a_{i}+p_{j}+t_{k}+x_{i j} \ell_{i}
$$

onde $\mu$ : média geral

$a_{i}$ : efeito do $i$-ésimo animal $(i=1,2, \ldots$, a)

$\mathrm{p}_{\mathrm{j}}$ : efeito do $\mathrm{j}$-ésimo periodo $(\mathrm{j}=1,2,3)$

$t_{k}$ : efeito do $k$-ésimo tratamento $(k=1,2, \ldots, k)$

$\ell_{i}$ : efeito linear de período $x$ animal, com

$$
x_{i j}=\left\{\begin{aligned}
-1 & \text { se } j=1 \\
0 & \text { se } j=2 \\
1 & \text { se } j=3, \quad \text { para todo } i .
\end{aligned}\right.
$$


Nestes trabalhos são apresentadas explicitamente as expressões das somas de quadrados, bem como as esperanças matemäticas dos quadrados médios, pa ra o caso particular de $\mathrm{K}=3$ tratamentos.

Segundo PATTERSON e LUCAS (1962), nenhum delineamento do tipo reversão è suficientemente capaz de prover boas estimativas dos chä mados efeitos residuais de um tratamento aplicado em um dado período so bre o periodo subseqüente. Nestas condições, a ünica solução para os en saios de reversão, quando há efeitos residuais de tratamentos, è despre zar as produções iniciais de cada período. Como esses são apenas três é aconselhável basear as interpretações sobre as produções de períodos de 4 a 5 semanas, após ter-se desprezado uma ou duas semanas.

\subsubsection{Delineamentos rotativos ("rotational")}

o tipo mais simples de delineamento rotativo é o quadrado 1atino ou um grupo de quadrados latinos, com linhas representando perío dos de observação e colunas representando as seqüencias de tratamentos.

Segundo PATTERSON (1950), quando não existe efeito residual de tratamentos, o método de anälise segundo tal delineamento como apresen tado em COCHRAN e COX (1957), conduz a estimativa não viciada do erro expe rimental, desde que se proceda a uma correta aleatorização das seqüèncias ou animais e dos tratamentos nas seqüencias.

Entretanto, em geral, considera-se a possibilidade de que tais efeitos residuais existam e deseja-se estimá-1os além dos efeitos di retos de tratamentos.

Neste sentido, COCHRAN et alii (1941) utilizam conjuntos completos de quadrados latinos ortogonais, objetivando um balanceamento desejado para melhorar a eficiência da anālise estatística, abordando es 
pecificamente $o$ caso em que $K=3$ e 4 tratamentos. Estes delineamentos são balanceados no sentido de que qualquer tratamento é precedido um igual nü mero de vezes $(\Lambda / 2)$ por cada um dos outros tratamentos.

Wi11ians (1949), citado em PATTERSON (1950), mostra que ba lanceamento desse tipo pode ser obtido com um ünico quadrado latino para um nümero par de tratamentos, e com dois quadrados latinos para um número impar de tratamentos.

Entretanto, exitem certas restrições ao uso de delineamen tos rotativos com $\mathrm{p}=\mathrm{K}$, devido às limitações em relação ao número de pe ríodos, uma vez que estes devem ser, segundo LUCAS (1960), de tamanho ra zoävel (4 a 5 semanas), o que os torna, para ensaios de uma única lacta ção, 1 imitados a de 3 a 5 .

Segundo PATTERSON (1950), é possivel encontrar esquemas convenientes de tratamentos com $\mathrm{p}<\mathrm{K}$, simplesmente considerando as $\mathrm{p} 1 \underline{i}$ nhas correspondentes de cada um dos $\mathrm{K}-1$ quadrados de um conjunto de qua drados latinos ortogonais $\mathrm{K} \times \mathrm{K}$, formando os denominados retāngulos 1 ati nos.

Nesse sentido são úteis os delineamentos construidos por PATTERSON (1952) e esquematizados em PATTERSON e LUCAS (1962) para $\mathrm{K}=3$, $4, \ldots, 16,18,21,26$ e 36 tratamentos subdivididos em quatro categorias, ou seja:
a) delineamentos rotativos balanceados
b) delineamentos rotativos parcialmente balanceados
c) delineamentos balanceados com período extra
d) delineamentos parcialmente balanceados com periodo extra, 


$$
\begin{aligned}
& \mathrm{p} \leq 6 \\
& \mathrm{k} \geq \mathrm{t} \geq \mathrm{p}-1 \\
& \mathrm{a}=\mathrm{bt} \leq 6 \mathrm{~K}<100,
\end{aligned}
$$

onde b é o número de blocos; $t$ o número de animais em cada bloco e a o nú mero total de animais ou seqüencias de tratamentos.

Os delineamentos balanceados são caracterizados pela apre sentação de todos os contrastes de tratamentos com estimação de igual pre cisão, bem como efeitos direto e residual de um dado tratamento negativa mente correlacionados. Para os parcialmente balanceados, alguns contras tes de tratamentos apresentam grau de precisão na sua estimação, diferen te de outros, ainda com correlação negativa entre efeitos direto e resi dual. Já os delineamentos com período extra são obtidos simplesmente re petindo-se o período final de um delineamento balanceado ou parcialmente balanceado. Segundo os autores, se os efeitos residuais são despresíveis, os delineamentos balanceados e os parcialmente balanceados são mais efi cientes do que os com período extra, os quais, por sua vez, são mais ef cientes que os primeiros se os efeitos residuais são de importância na ex perimentação.

Segundo os autores, todo delineamento "Change-over" com $\mathrm{K}$ tratamentos e p períodos $(2 \leq \mathrm{p} \leq \mathrm{K})$ utilizando a animais, subdivididos em b blocos, com t animais por bloco, e dito balanceado, se quaisquer dois tratamentos $k$ e $k^{\prime}\left(k \neq k^{\prime}\right)$ satisfazem as condições:

1) tratamento $\mathrm{k}$ ocorre em cada periodo $\frac{\mathrm{a}}{\mathrm{k}}$ vezes;

2) tratamentos $k$ e $k^{\prime}$ ocorrem juntos em $\frac{a(t-1)}{k(k-1)}$ blocos em cada período;

3) se os tratamentos $k$ e $k^{\prime}$ ocorrem juntos em um dado blo 
co em qualquer periodo, então eles tambén ocorrem juntos em todos os ou tros períodos;

4) cada animal recebe um dado tratamento uma ünica vez;

5) tratamento $\mathrm{k}$ é imediatamente precedido pelo tratamento $k^{\prime}$ em $\frac{\Lambda}{2}=\frac{a(p-1)}{k(k-1)}$ seqüencias;

6) tratamentos $k$ e $k^{\prime}$ ocorrem juntos em $\frac{a p(p-1)}{k(K-1)}$ seqüencias;

7) tratamento $k$ ocorre em $\frac{a(p-1)}{K(K-1)}$ das $\frac{a}{K}$ seqüèncias, nas quais o tratamento $\mathrm{k}^{\prime}$ ocorre no p-ésimo período.

No presente trabalho, embora não seja abordada a existēn cia de efeitos residuais de tratamentos, serão considerados na metodolo gia os delineamentos balanceados com $\mathrm{p} \leq \mathrm{K}$ apresentados em PATTERSON e LUCAS (1962), uma vez que uma seqüência lógica de pesquisa serà a poste rior consideração de tais efeitos.

\subsection{Modelo auto-regressivo em ensaios de lactação}

GODOI (1983) é o primeiro a utilizar o modelo auto-regres sivo, cuja estrutura de correlação é dada em (1), para análise de dados de produção de leite, abordando especificamente uma confrontação com o en saio de reversão. Neste trabalho o autor faz uso do mesmo desenho experi mental proposto por LUCAS (1956), porém com a adoção do modelo auto-regres sivo, e verifica uma maior sensibilidade desse modelo na deteção de con trastes de efeitos de tratamentos através do teste F. Entretanto, tal sen sibilidade só é maior nos casos em que o coeficiente de correlação linear entre períodos é alto $(\rho \geq 0,70)$, indicando assim que, se os animais amos trados para o ensaio estiverem com produções homogêneas, o modelo prefe rivel seja o de reversão, jä que tal coeficiente è dependente da variabi 
lidade entre animais. Ainda neste trabalho o autor aventa a possibilida de de que os delineamentos em blocos incompletos ou ainda os rotativos se jam mais eficientes ou sensiveis, se incorporarmos a estes uma estrutura de correlação realista, ou seja a auto-regressiva.

Os resultados obtidos por Godoi, bem. como suas sugestões, è que motivam preponderantemente a realização deste trabalho. Desta for ma cuida-se de utilizar os delineamentos rotativos balanceados com $\mathrm{p} \leq \mathrm{K}$ de PATTERSON e LUCAS (1962) e, com a adoção do modelo matemático (2) com estrutura de correlação (3), estudar a sensibilidade deste modelo, compa rada com os de métodos usuais de análise de ensaios com vacas em lactação. Assim, para o modelo 1 inear geral

$$
\underline{\mathrm{Y}}=\underset{\sim}{\mathrm{X} \beta}+\underline{\mathrm{e}}
$$

onde $\quad E(\underset{\sim}{e})=\underset{\sim}{0} ; \quad E\left(\underset{\sim}{e} e_{\sim}^{\prime}\right)=V \sigma_{e}^{2}$

com V simétrica e definida positiva, definida em (3), temos dois casos a considerar.

Inicialmente se $\mathrm{V}$ for conhecida, como é abordada no presen te trabalho, autores como RAO (1965), THEIL (1971) ou HOFFMANN e VIEIRA (1977) mostram que uma simples transformação linear não singular, nos le va à aplicação do método dos mínimos quadrados usual para a obtenção do estimador linear imparcial de variāncia minima do vetor de parämetros $\underset{\sim}{\beta}$. Um resultado fundamental è que tal estimador apresenta as mesmas proprie dades dos estimadores obtidos quando se trabalha com erros independentes e homocedásticos.

No caso em que $V$ é desconhecida, isto é, quando $\rho$ é desco nhecido, pode-se adotar o procedimento recomendado por THIL (1971) que mo 
dificado é utilizado por GODOI (1983), o qual consiste na obtenção de uma estimativa $\bar{\rho}$, através dos residuos $\left(y_{i j k}-\bar{y}_{i j k}\right)$, por um processo iterati vo de estabilização do valor de $\hat{\rho}$. Entretanto, verifica-se que a mera substituição de $\rho$ por $\hat{\rho}$, e a aplicação do método dos mínimos quadrados, acarreta uma tendencia indevida do modelo auto-regressivo de rejeitar a hipótese de igualdade de efeitos de tratamentos.

Ademais, não se pode descartar a possibilidade de que a fonte dos dados a serem utilizados em ensaios desta natureza, quer sejam institutos de pesquisa ou fazendas experimentais, disponham de arquivos com as produções de um grande número de animais, dos quais uma parte será amostrada para a realização do experimento. Assim, do conjunto total, po de ser obtido um valor para o coeficiente de correlação linear entre pe ríodos, tomado de forma coerente com o ensaio, sob a hipótese de que tal valor não sofra grandes alterações para lactações consecutivas. 


\section{MATERIAL E MÉTODOS}

Para a comparação da sensibilidade na deteç̧ão de contras tes de efeitos de tratamentos pelos modelos: auto-regressivo, de reversão e rotativo, serão utilizados os dados da Tabela 1, os quais correspondem a médias quinzenais de produção de leite de 144 vacas da raça holandeza, sendo que a produção do primeiro periodo é tomada após o pico da lactação, de modo que os sete periodos considerados se encontram no intervalo de produção útil para experimentos desta natureza. A fonte destes dados é - Departamento de Zootecnia da ESALQ-USP, Setor de Ruminantes, os quais foram obtidos e trabalhados inicialmente por GODOI (1971), apresentados em GODOI (1983), com o qual estamos em débito pela permissão para a utiliza ção dos mesmos.

Em se calculando a correlação linear entre períodos, cuja ordem de grandeza é dependente da variabilidade de produção entre vacas, obtém-se a Tabela 2, na qual pode-se observar uma constância nas correla ções entre períodos separados por um intervalo fixo. Por outro lado, atra vẻs da matriz de correlações do modelo auto-regressivo (1) considerandose $\rho=0,91$, que é a média das correlações entre períodos consecutivos, ob 
tém-se a Tabela 3, donde se pode perceber uma boa adaptação desta estrutü ra de correlações aos dados originais.

Consideram-se três ensaios, variando o nümero de tratamen tos, ou seja: $K=3,4$ e 5, o que nos dá uma desejável variação do número de graus de liberdade para o residuo dos modelos considerados. Sendo es te um estudo comparativo, adota-se o mesmo nümero de animais ou seqüencias de tratamentos para todos os modelos.

Para cada ensaio considera-se cinco situações para os efei tos de tratamentos $t_{k}$, os quais, alēm do efeito nulo, variam de $2 \%$ a $8 \%$ da produção média (10 kg/dia) obtida da Tabela 1 . Os ensaios considera dos são esquematizados a seguir:

a) Ensaio 1: $\mathrm{K}=3$ tratamentos

a.1) Esquema do ensaio para o modelo de reversão completo

\begin{tabular}{|c|c|c|c|c|c|c|}
\hline \multirow{2}{*}{ Períodos } & \multicolumn{2}{|c|}{ Animais } & \multicolumn{3}{|c|}{ (Seqüëncias de Tratamentos) } & \multirow[b]{2}{*}{6} \\
\hline & 1 & 2 & 3 & 4 & 5 & \\
\hline I & (1) & (2) & (3) & (1) & (2) & (3) \\
\hline II & (2) & (3) & (1) & (3) & (1) & (2) \\
\hline III & (1) & (2) & (3) & (1) & (2) & (3) \\
\hline
\end{tabular}

Esquema da anālise da variāncia segundo LUCAS (1956)

\begin{tabular}{lcc}
\hline F. Variação & G.L. & G.L. Caso Geral \\
\hline Tratamentos & 2 & $\mathrm{~K}-1$ \\
Resíduo & 3 & $(\mathrm{~K}-1)^{2}-1$ \\
\hline Total & 5 & $\mathrm{~K}(\mathrm{~K}-1)-1$ \\
\hline
\end{tabular}


a.2) Esquema do ensaio para o modelo rotativo com dois quadrados lati nos (Q.L.)

\begin{tabular}{ccccccc} 
Periodos & \multicolumn{7}{c}{ Animais } \\
\cline { 2 - 7 } & 1 & 2 & 3 & 4 & 5 & 6 \\
\hline I & $(1)$ & $(2)$ & $(3)$ & $(1)$ & $(2)$ & $(3)$ \\
II & $(2)$ & $(3)$ & $(1)$ & $(3)$ & $(1)$ & $(2)$ \\
III & $(3)$ & $(1)$ & $(2)$ & $(2)$ & $(3)$ & $(1)$ \\
\hline \hline
\end{tabular}

Esquema da anālise da variância segundo COCHRAN e COX (1957)

\begin{tabular}{lll}
\hline F. Variação & G.L. & G.L. Caso Geral \\
\hline Quadrados Latinos (Q.L.) & 1 & $\mathrm{q}-1$ \\
Períodos d. Q.L. & 4 & $\mathrm{q}(\mathrm{K}-1)$ \\
Animais d. Q.L. & 4 & $\mathrm{q}(\mathrm{K}-1)$ \\
Tratamentos (T) & 2 & $\mathrm{~K}-1$ \\
Interação $\mathrm{T} \times$ Q.L. & 2 & $(\mathrm{q}-1)(\mathrm{K}-1)$ \\
Residuo & 4 & $\mathrm{q}(\mathrm{K}-1)(\mathrm{K}-2)$ \\
Resíduo Médio & 6 & $\mathrm{q}(\mathrm{K}-1)-(\mathrm{K}-1)$ \\
\hline Total & 17 & $\mathrm{qK}-1$ \\
\hline \hline
\end{tabular}

I/o Resíduo médio (Resíduo + Interação $\mathrm{T} \times$ Q.L.) serả utilizado caso a interação $T \times$ Q.L. seja não significativia.

a.3) Esquema do ensaio para o modelo auto-regressivo segundo PATTERSON e LUCAS (1962)

\begin{tabular}{cccccccc}
\hline & \multicolumn{7}{c}{ Animais } \\
\cline { 2 - 7 } Periodos & 1 & 2 & 3 & 4 & 5 & 6 \\
\hline I & $(1)$ & $(2)$ & $(3)$ & $(1)$ & $(2)$ & $(3)$ \\
II & $(2)$ & $(3)$ & $(1)$ & $(3)$ & $(1)$ & $(2)$ \\
III & $(3)$ & $(1)$ & $(2)$ & $(2)$ & $(3)$ & $(1)$ \\
\hline \hline
\end{tabular}


Esquema da anālise da variancia

\begin{tabular}{lcc}
\hline F. Variação & G.L. & G.L. Caso Geral \\
\hline Periodos & 2 & $\mathrm{p}-1$ \\
Tratamentos & 2 & $\mathrm{~K}-1$ \\
Residuo & 13 & $\mathrm{p}(\mathrm{a}-1)-(\mathrm{K}-1)$ \\
\hline Total & 17 & $\mathrm{ap}-1$ \\
\hline \hline
\end{tabular}

onde $\mathrm{a}=$ número de animais.

Valores considerados para os efeitos de tratamentos $t_{k}$

1) $t_{k}=0, k=1,2,3$

2) $t_{1}=0,2 ; \quad t_{2}=-0,2 ; \quad t_{3}=0,0$

3) $t_{1}=0,4 ; t_{2}=-0,4 ; t_{3}=0,0$

4) $t_{1}=0,6 ; \quad t_{2}=-0,6 ; t_{3}=0,0$

5) $t_{1}=0,8 ; \quad t_{2}=-0,8 ; t_{3}=0,0$.

b) Ensaio 2. $K=4$ tratamentos

b.1) Esquema do ensaio para o modelo de reversão completo

\begin{tabular}{|c|c|c|c|c|c|c|c|c|c|c|c|c|}
\hline \multirow{2}{*}{ Períodos } & \multicolumn{12}{|c|}{ Animais } \\
\hline & 1 & 2 & 3 & 4 & 5 & 6 & 7 & 8 & 9 & 10 & 11 & 12 \\
\hline I & (1) & (2) & (3) & (4) & (1) & $(2)$ & (3) & (4) & (1) & (2) & (3) & (4) \\
\hline II & (2) & (3) & (4) & (1) & (3) & (4) & (1) & (2) & (4) & (1) & (2) & (3) \\
\hline III & (1) & (2) & (3) & (4) & (1) & (2) & (3) & $(4)$ & (1) & (2) & (3) & (4) \\
\hline
\end{tabular}

com $8 \mathrm{~g} \cdot 1$. para o resíduo. 
b.2) Esquema do ensaio para o modelo rotativo com 3 Q.L.

\begin{tabular}{cccccccccccccc}
\hline \hline & \multicolumn{11}{c}{ Animais } \\
\cline { 2 - 12 } & 1 & 2 & 3 & 4 & 5 & 6 & 7 & 8 & 9 & 10 & 11 & 12 \\
\hline & $(1)$ & $(2)$ & $(3)$ & $(4)$ & $(1)$ & $(2)$ & $(3)$ & $(4)$ & $(1)$ & $(2)$ & $(3)$ & $(4)$ \\
I & II & $(2)$ & $(3)$ & $(4)$ & $(1)$ & $(2)$ & $(3)$ & $(4)$ & $(1)$ & $(2)$ & $(3)$ & $(4)$ & $(1)$ \\
III & $(4)$ & $(1)$ & $(2)$ & $(3)$ & $(4)$ & $(1)$ & $(2)$ & $(3)$ & $(4)$ & $(1)$ & $(2)$ & $(3)$ \\
IV & $(3)$ & $(4)$ & $(1)$ & $(2)$ & $(3)$ & $(4)$ & $(1)$ & $(2)$ & $(3)$ & $(4)$ & $(1)$ & $(2)$ \\
\hline \hline
\end{tabular}

com $18 \mathrm{~g} .1$. para o resíduo e $24 \mathrm{~g} .1$. para o resíduo médio.

b.3) Esquema do ensaio para o modelo auto-regressivo com $p=3$ periodos

\begin{tabular}{rllllllllllllll}
\hline \hline & \multicolumn{11}{c}{ Animais } \\
\cline { 2 - 12 } & 1 & 2 & 3 & 4 & 5 & 6 & 7 & 8 & 9 & 10 & 11 & 12 \\
\hline Periodos & $(1)$ & $(2)$ & $(3)$ & $(4)$ & $(1)$ & $(2)$ & $(3)$ & $(4)$ & $(1)$ & $(2)$ & $(3)$ & $(4)$ \\
II & $(2)$ & $(3)$ & $(4)$ & $(1)$ & $(3)$ & $(4)$ & $(1)$ & $(2)$ & $(4)$ & $(1)$ & $(2)$ & $(3)$ \\
III & $(3)$ & $(4)$ & $(1)$ & $(2)$ & $(4)$ & $(1)$ & $(2)$ & $(1)$ & $(2)$ & $(3)$ & $(4)$ & $(1)$ \\
\hline \hline
\end{tabular}

com 30 g.1. para o resíduo.

b.4) Esquema do ensaio para o modelo auto-regressivo com $p=K$ 0 mesmo de b.2 com $41 \mathrm{~g} .1$. para o residuo.

Valores considerados para os efeitos de tratamentos $t_{k}$

1) $t_{k}=0, k=1,2,3,4$

2). $t_{1}=0,2 ; \quad t_{2}=-0,2 ; \quad t_{3}=0,2 ; \quad t_{4}=-0,2$

3) $t_{1}=0,4 ; \quad t_{2}=-0,4 ; \quad t_{3}=0,4 ; \quad t_{4}=-0,4$

4) $t_{1}=0,6 ; t_{2}=-0,6 ; t_{3}=0,6 ; t_{4}=-0,6$

5) $t_{1}=0,8 ; t_{2}=-0,8 ; \quad t_{3}=0,8 ; \quad t_{4}=-0,8$. 
c) Ensaio 3. $K=5$ tratamentos

c.1) Esquema do ensaio para o modelo de reversão completo

\begin{tabular}{|c|c|c|c|c|c|c|c|c|c|c|c|c|}
\hline \multirow{2}{*}{ Períodos } & \multicolumn{12}{|c|}{ Animais } \\
\hline & 1 & 2 & 3 & 4 & 5 & 6 & 7 & 8 & 9 & 10 & $\begin{array}{lllll}11 & 12 & 13 & 14 & 15\end{array}$ & $\begin{array}{lllll}16 & 17 & 18 & 19 & 20\end{array}$ \\
\hline I & (1) & (2) & (3) & (4) & (5) & (1) & (2) & (3) & & (5) & $(1)(2)(3)(4)(5)$ & $(1)(2)(3)(4)(5)$ \\
\hline II & (2) & (3) & (4) & & (1) & (3) & (4) & (5) & & (2) & $(4)(5)(1)(2)(3)$ & $(5)(1)(2)(3)(4)$ \\
\hline $\operatorname{III}$ & (1) & (2) & (3) & (4) & (5) & (1) & (2) & (3) & (4) & (5) & $(1)(2)(3)(4)(5)$ & $(1)(2)(3)(4)(5)$ \\
\hline
\end{tabular}

com $15 \mathrm{~g} \cdot 1$. para o residuo.

c.2) Esquema do ensaio para o modelo rotativo com 4 Q.L.

Animais

$\begin{array}{llllllllllllllllllll}1 & 2 & 3 & 4 & 5 & 6 & 7 & 8 & 9 & 10 & 11 & 12 & 13 & 14 & 15 & 16 & 17 & 18 & 19 & 20\end{array}$

I (1)(2)(3)(4)(5) (1)(2)(3)(4)(5) (1)(2)(3)(4)(5) (1) (2) (3) (4) (5)

II (2)(3)(4)(5)(1) (3)(4)(5)(1)(2) (2)(3)(4)(5)(1) (3)(4)(5)(1)(2)

III (4)(5)(1)(2)(3) (2)(3)(4)(5)(1) (4)(5)(1)(2)(3) (2)(3) (4) (5) (1)

IV $\quad(5)(1)(2)(3)(4)(5)(1)(2)(3)(4)(5)(1)(2)(3)(4) \quad(5)(1)(2)(3)(4)$

$\mathrm{V} \quad(3)(4)(5)(1)(2)(4)(5)(1)(2)(3)(3)(4)(5)(1)(2) \quad(4)(5)(1)(2)(3)$

com $48 \mathrm{~g} .1$. para o resíduo e $60 \mathrm{~g} .1$. para o residuo médio.

c.3) Esquema do ensaio para o modelo auto-regressivo com $p=3$ períodos

Periodos Animais

\begin{tabular}{llllllllllllllllllll}
1 & 2 & 3 & 4 & 5 & 6 & 7 & 8 & 9 & 10 & 11 & 12 & 13 & 14 & 15 & 16 & 17 & 18 & 19 & 20 \\
\hline
\end{tabular}

I (1) (2)(3)(4)(5) (1)(2)(3)(4)(5)(1)(2)(3)(4)(5) (1)(2)(3) (4) (5)

II (2) (3)(4)(5)(1) (3)(4)(5)(1)(2) (4)(5)(1)(2)(3) (5) (1) (2) (3) (4)

III (3) (4)(5)(1)(2) (5)(1)(2)(3)(4)(2)(3)(4)(5)(1) (4) (5)(1)(2)(3)

com $53 \mathrm{~g} .1$. para o resíduo. 
c.4) Esquema do ensaio para o modelo auto-regressivo com $p=k$ 0 mesmo de c.2 com $91 \mathrm{~g} .1$. para o residuo.

Valores considerados para os efeitos de tratamentos $t_{k}$

1) $\mathrm{t}_{\mathrm{k}}=0, \mathrm{k}=1,2,3,4,5$

2) $t_{1}=0,2 ; \quad t_{2}=-0,2 ; \quad t_{3}=0,2 ; \quad t_{4}=-0,2 ; \quad t_{5}=0,0$

3) $t_{1}=0,4 ; \quad t_{2}=-0,4 ; t_{3}=0,4 ; \quad t_{4}=-0,4 ; \quad t_{5}=0,0$

4) $t_{1}=0,6 ; \quad t_{2}=-0,6 ; \quad t_{3}=0,6 ; \quad t_{4}=-0,6 ; \quad t_{5}=0,0$

5) $t_{1}=0,8 ; \quad t_{2}=-0,8 ; \quad t_{3}=0,8 ; t_{4}=-0,8 ; \quad t_{5}=0,0$.

Com o objetivo de verificar se pequenas alterações no va lor populacional do coeficiente de correlação linear entre períodos, acar retam diferenças na sensibilidade do teste de significância ou algum tipo de viés nas estatisticas envolvidas nos testes, considera-se para o mode 10 auto-regressivo em cada ensaio, os seguintes valores de $\rho: 0,88 ; 0,90$; 0,$91 ; 0,92 ; 0,94$

Para cada ensaio são efetuadas 100 amostragens de animais e periodos, entre os dados da Tabela 1 , e obtidas as respectivas anälises da variancia para os diferentes valores de $t_{k}$, e $\rho$ no caso do modelo au to-regressivo.

Das anälises da variāncia são considerados os valores da estatistica $F$ para tratamentos, e para cada valor desta estatistica deter mina-se o nível mínimo de significāncia (N.M.S.) com auxílio da função subprograma FF, adaptada da sub-rotina PF apresentada em KENNEDY e GENTLE (1980). Segundo MOOD et alii (1974), quando se considera $t_{k}=0, k=1$, $2, \ldots, K$, os N.M.S. em cada ensaio serão uniformemente distribuídos no intervalo $(0,1)$. Determina-se então, para cada ensaio, a distribuição de 
freqüència dos 100 N.M.S. no interva10 $(0,1)$ em classes de amplitude 0,05 , bem como efetua-se o teste de aderéncia ponto a ponto de Kolmogorov-Smir nov.

Nos casos em que $t_{k} \neq 0$ para algum $k=1,2, \ldots, k, \quad$ os N.M.S. são calculados com o objetivo de se verificar qual modelo apresen ta maior sensibilidade para detectar diferenças entre tais efeitos, bem como a influência do valor de $\rho$ nesta sensibilidade no caso do modelo ser - auto-regressivo. O modelo mais sensivel serä aquele para o qual a dis tribuição dos N.M.S. se concentrar nas classes iniciais do intervalo $(0,1)$. Por outro lado, em se conhecendo as expressões das esperan ças matemáticas dos quadrados médios para tratamentos e resíduo no modelo auto-regressivo, utiliza-se a média dos valores da estatística $F$, bem co mo o quociente de médias do Q.M. tratamentos pelo Q.M. resíduo, como uma forma de controle da adequação desse modelo às condições experimentais. 
4. RESULTADOS E DISCUSSÃO

4.1. Anālise da variância no modelo auto-regressivo

4.1.1. O modelo linear geral

$$
\begin{aligned}
& \text { Para o modelo } \underset{\sim}{\mathrm{Y}}=\mathrm{X}_{\sim}^{\mathrm{B}}+\underset{\sim}{\mathrm{e}} \text {, onde } \\
& \left.\underset{\sim}{\mathrm{Y}^{\prime}}=\left[\mathrm{y}_{11 \mathrm{k}_{11}} \ldots \mathrm{y}_{1 \mathrm{pk}}{ }_{1 \mathrm{p}} \mathrm{y}_{21 \mathrm{k}_{21}} \cdots \mathrm{y}_{2 \mathrm{pk}} \ldots \mathrm{y}_{\mathrm{apk}}\right]_{\mathrm{ap}}\right]_{(1 \times \mathrm{ap})}
\end{aligned}
$$

com: $\quad y_{i j k}=$ observação no $i$-ésimo animal $(i=1,2, \ldots$, a) que no $j$-ési mo período $(j=1,2, \ldots, p)$ recebeu o tratamento $k(k=1,2, \ldots, k)$; $p \leq k$ e $k_{i j} \neq k_{i j}$, para $j \neq j^{\prime}$,

$$
\begin{aligned}
& \underline{\beta}^{\prime}=\left[\mu: p_{1} p_{2} \ldots p_{p}: t_{1} t_{2} \ldots t_{K}\right]_{(1 \times n)} ; n=1+p+K, \\
& \mathrm{X}=\text { matriz de incidēncia de ordem ap } \times \mathrm{n} \text {, } \\
& \underline{\mathbf{e}}=\text { vetor de erros de ordem ap } \times 1 \text {, com } \\
& \mathrm{E}(\underset{\sim}{\mathrm{e}})=\underset{\sim}{0} ; \quad \operatorname{Var}(\underset{\sim}{\mathrm{e}})=\sigma_{\mathrm{e}}^{2} \mathrm{~V}
\end{aligned}
$$


para $V$ simétrica e definida positiva, definida em (3), temos que

$$
\begin{aligned}
& \operatorname{Var}(\underset{\sim}{Y})=\sigma_{e}^{2} V=\left(I_{a} \otimes A\right) \sigma_{e}^{2}, \operatorname{com} \\
& \left(I_{a} \otimes A\right)=\left[\begin{array}{llllll}
A & \phi & . & . & \phi \\
\phi & A & \cdot & \cdots & \phi \\
\ldots & \ldots & \ldots & \ldots & \ldots \\
\phi & \phi & . & . & A
\end{array}\right]_{(\text {apxap })}
\end{aligned}
$$

com $\phi$ a matriz nula de ordem $p \times p$,

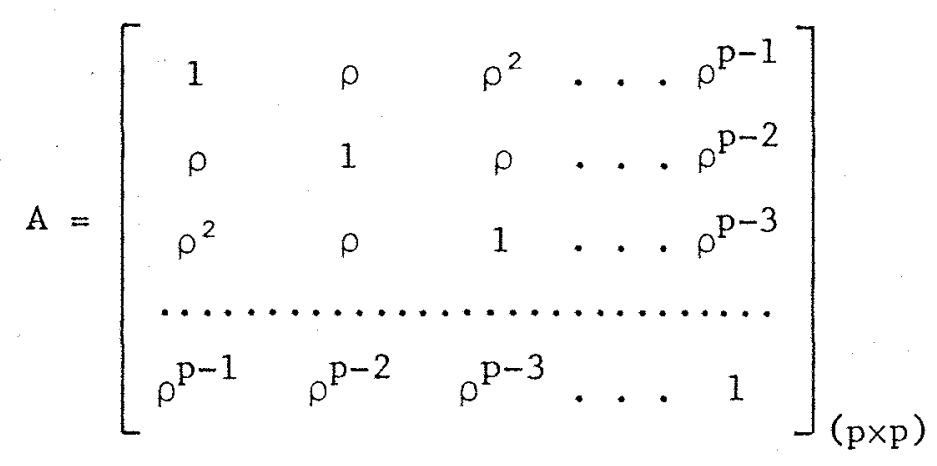

e $\mathrm{I}_{\mathrm{a}}$ a matriz identidade de ordem a

Para $|\rho|<1$ a matriz A é definida positiva e

$$
A^{-1}=\frac{1}{1-\rho^{2}}\left[\begin{array}{cccccc}
1 & -\rho & 0 & . & . & 0 \\
-\rho & 1+\rho^{2} & -\rho & . & . & 0 \\
0 & -\rho & 1+\rho^{2} & . & . & 0 \\
\ldots & \ldots & \ldots & \ldots & \ldots & \ldots \\
0 & 0 & 0 & . & . & 1
\end{array}\right]
$$

Sendo A simétrica e definida positiva, $A^{-1}$ tambēm o serä e portanto existe uma matriz não singular $D$ tal que

$$
D^{\prime} D=A^{-1} \Leftrightarrow D^{-1}\left(D^{\prime}\right)^{-1}=A
$$


Seja então:

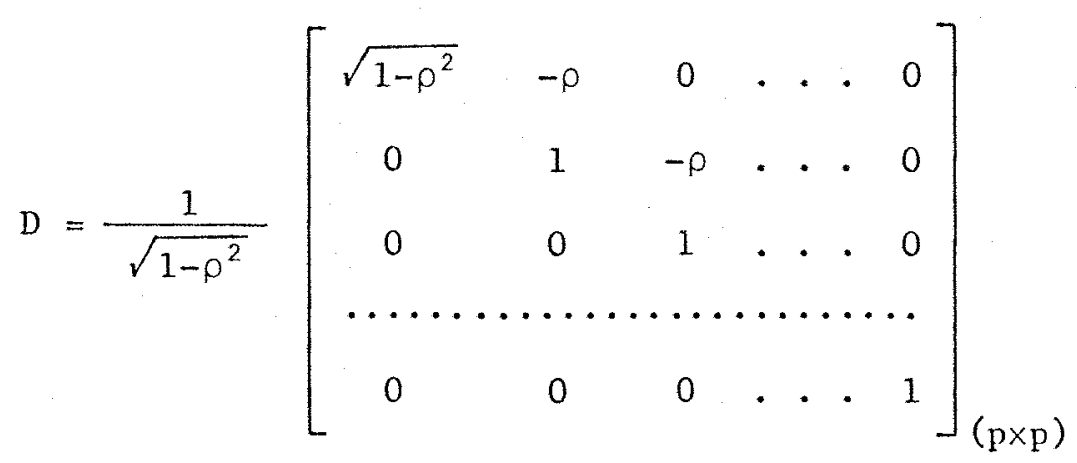

satisfazendo (4) e seja a transformação não singular

$$
\underset{\sim}{Y}(\rho)=\left(I_{a} \otimes D^{\prime}\right) \underset{\sim}{Y}
$$

onde $\underset{\sim}{Y}$ é o vetor de observações originais e $\underset{\sim}{Y}(\rho)$ o vetor de observações transformadas. Então:

$$
E[\underset{\sim}{Y}(\rho)]=\left(I_{a} \otimes D^{\prime}\right) \underset{\sim}{X}
$$

e

$$
\operatorname{Var}[\underset{\sim}{Y}(\rho)]=\left(I_{a} \otimes D^{\prime}\right) \operatorname{Var} \underset{\sim}{(Y)}\left(I_{a} \otimes D^{\prime}\right)^{\prime}
$$

ou

$$
\operatorname{Var}[\underline{\sim}(\rho)]=\left(I_{a} \otimes D^{\prime}\right) \cdot\left(I_{a} \otimes A\right)\left(I_{a} \otimes D^{\prime}\right)^{\prime} \sigma_{e}^{2}
$$

Observando que $(A \otimes B)^{\prime}=A^{\prime} \otimes B^{\prime}$ e $(A \otimes B) .(C \otimes D)=A C \otimes B D$ temos pela expressão (4) que

$$
\begin{aligned}
\operatorname{Var}[\underset{\sim}{\mathrm{Y}}(p)] & =\left(I_{a} \otimes D^{\prime}\right)\left[I_{a} \otimes\left(D^{\prime}\right)^{-1} D^{-1}\right]\left(I_{a} \otimes D\right) \sigma_{e}^{2} \\
& =\left[I_{a} \otimes D^{\prime}\left(D^{\prime}\right)^{-1} D^{-1} D\right] \sigma_{e}^{2} \\
& =\left(I_{a} \otimes I_{p}\right) \sigma_{e}^{2}
\end{aligned}
$$

Logo:

$$
\operatorname{Var}[\underset{\sim}{Y}(\rho)]=I_{\text {ap }} \sigma_{e}^{2}
$$


ou seja, através da transformação não singular (5) passamos de um modelo linear com dispersão $V \sigma_{\mathrm{e}}^{2}$ para outro também linear, porém com dispersão simplificada $I \sigma_{e}^{2}$. Portanto, as funções estimäveis segundo ambos os mode los, são as mesmas.

\subsubsection{Sistema de equações normais}

Se C' $\underset{\sim}{\beta}$ é estimável, então o estimador linear imparcial de

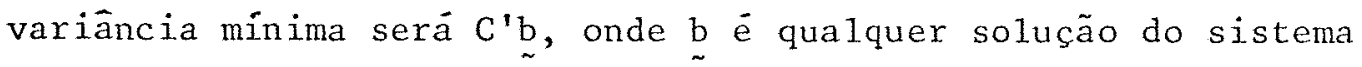

$$
\left[\left(I_{a} \otimes D^{\prime}\right) X\right]^{\prime}\left[\left(I_{a} \otimes D^{\prime}\right) X\right] \underset{\sim}{b}=\left[\left(I_{a} \otimes D^{\prime}\right) X\right]^{\prime} \underset{\sim}{Y}(\rho)
$$

Usando (5) vem

$$
\left[X^{\prime}\left(I_{a} \otimes D\right)\right]\left[\left(I_{a} \otimes D^{\prime}\right) X\right] \underset{\sim}{b}=\left[X^{\prime}\left(I_{a} \otimes D\right)\right]\left(I_{a} \otimes D^{\prime}\right) \underset{\sim}{Y}
$$

por (4) obtemos

$$
X^{\prime}\left(I_{a} \otimes A^{-1}\right) X_{\sim}=X^{\prime}\left(I_{a} \otimes A^{-1}\right) \underset{\sim}{Y}
$$

Com o objetivo de ilustrarmos a obtenção das matrizes $X^{\prime}\left(I_{a} \otimes A^{-1}\right) X$ e $X^{\prime}\left(I_{a} \otimes A^{-1}\right) Y$ no caso geral de $K$ tratamentos e $p$ períodos $(\mathrm{p} \leq \mathrm{k})$, consideremos o caso particular de um delineamento com $\mathrm{K}=\mathrm{p}=3$, cujo esquema balanceado é:

\begin{tabular}{ccccccc}
\hline & \multicolumn{7}{c}{ Animais } \\
\cline { 2 - 7 } & 1 & 2 & 3 & 4 & 5 & 6 \\
\hline Periodos & $(1)$ & $(2)$ & $(3)$ & $(1)$ & $(2)$ & $(3)$ \\
II & $(2)$ & $(3)$ & $(1)$ & $(3)$ & $(1)$ & $(2)$ \\
III & $(3)$ & $(1)$ & $(2)$ & $(2)$ & $(3)$ & $(1)$ \\
\hline
\end{tabular}


Neste caso temos:

$$
\begin{aligned}
& a=6 \text { animais ou seqüencias de tratamentos } \\
& r=6 \text { repetições de tratamentos } \\
& K=3 \text { tratamentos } \\
& \mathrm{p}=3 \text { periodos } \\
& \Lambda=4 \text { número de vezes que um tratamento } k \text { é precedido } \\
& \quad \text { e/ou sucedido por outro } k^{\prime} .
\end{aligned}
$$

Então

$$
\begin{aligned}
& \underset{\sim}{\beta^{\prime}}=\left[\mu: p_{1} p_{2} p_{3}: t_{1} t_{2} t_{3}\right]
\end{aligned}
$$

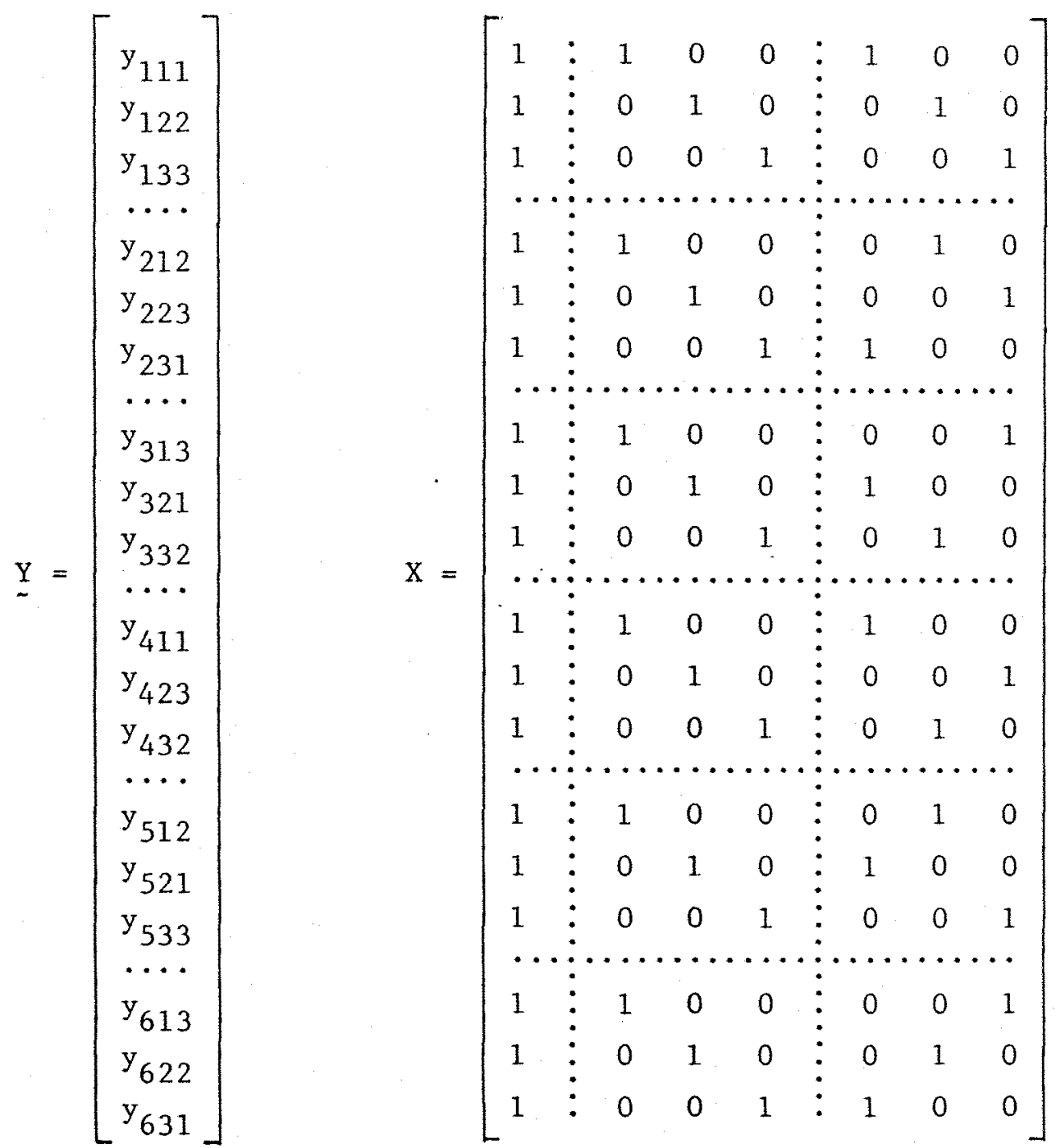


e obtemos as matrizes:

\begin{tabular}{|c|c|c|c|c|c|c|c|}
\hline & $\sqrt{1-\rho^{2}}$ & $\sqrt{1-\rho^{2}}$ & 0 & 0 & $: \sqrt{1-\rho^{2}}$ & 0 & 0 \\
\hline & $1-\rho$ & $-\rho$ & 1 & 0 & $-\rho$ & 1 & 0 \\
\hline & $1-\rho$ & 0 & $-\rho$ & 1 & 0 & $-\rho$ & 1 \\
\hline & $\sqrt{1-\rho^{2}}$ & $\sqrt{1-\rho^{2}}$ & 0 & 0 & 0 & $\sqrt{1-\rho^{2}}$ & 0 \\
\hline & $1-\rho$ & $-\rho$ & 1 & 0 & 0 & $-\rho$ & 1 \\
\hline & $1-\rho$ & 0 & $-\rho$ & 1 & 1 & 0 & $-\rho$ \\
\hline & $\sqrt{1-\rho^{2}}$ & $\sqrt{1-\rho^{2}}$ & 0 & 0 & 0 & 0 & $\sqrt{1-\rho^{2}}$ \\
\hline & $1-\rho$ & $-p$ & 1 & 0 & 1 & 0 & $-\rho$ \\
\hline 1 & $1-\rho$ & 0 & $-\rho$ & 1 & $-\rho$ & 1 & 0 \\
\hline$a-\sqrt{1-\rho^{2}}$ & $\sqrt{1-\rho^{2}}$ & $\sqrt{1-\rho^{2}}$ & 0 & 0 & $\sqrt{1-p^{2}}$ & 0 & 0 \\
\hline & $1-\rho$ & $-p$ & 1 & 0 & $-p$ & 0 & 1 \\
\hline & $1-\rho$ & 0 & $-\rho$ & 1 & 0 & 1 & $-\rho$ \\
\hline & $\sqrt{1-\rho^{2}}$ & $\sqrt{1-p^{2}}$ & 0 & 0 & 0 & $1-\rho^{2}$ & 0 \\
\hline & $1-\rho$ & $-\rho$ & 1 & 0 & 1 & $-\rho$ & 0 \\
\hline & $1-\rho$ & 0 & $-\rho$ & 1 & $-\rho$ & 0 & 1 \\
\hline & $\sqrt{1-\rho^{2}}$ & $\sqrt{1-p^{2}}$ & 0 & 0 & 0 & 0 & $\sqrt{1-\rho^{2}}$ \\
\hline & $1-\rho$ & $-\rho$ & 1 & 0 & 0 & 1 & $-\rho$ \\
\hline & $1-\rho$ & 0 & $-\rho$ & 1 & 1 & $-p$ & 0 \\
\hline
\end{tabular}




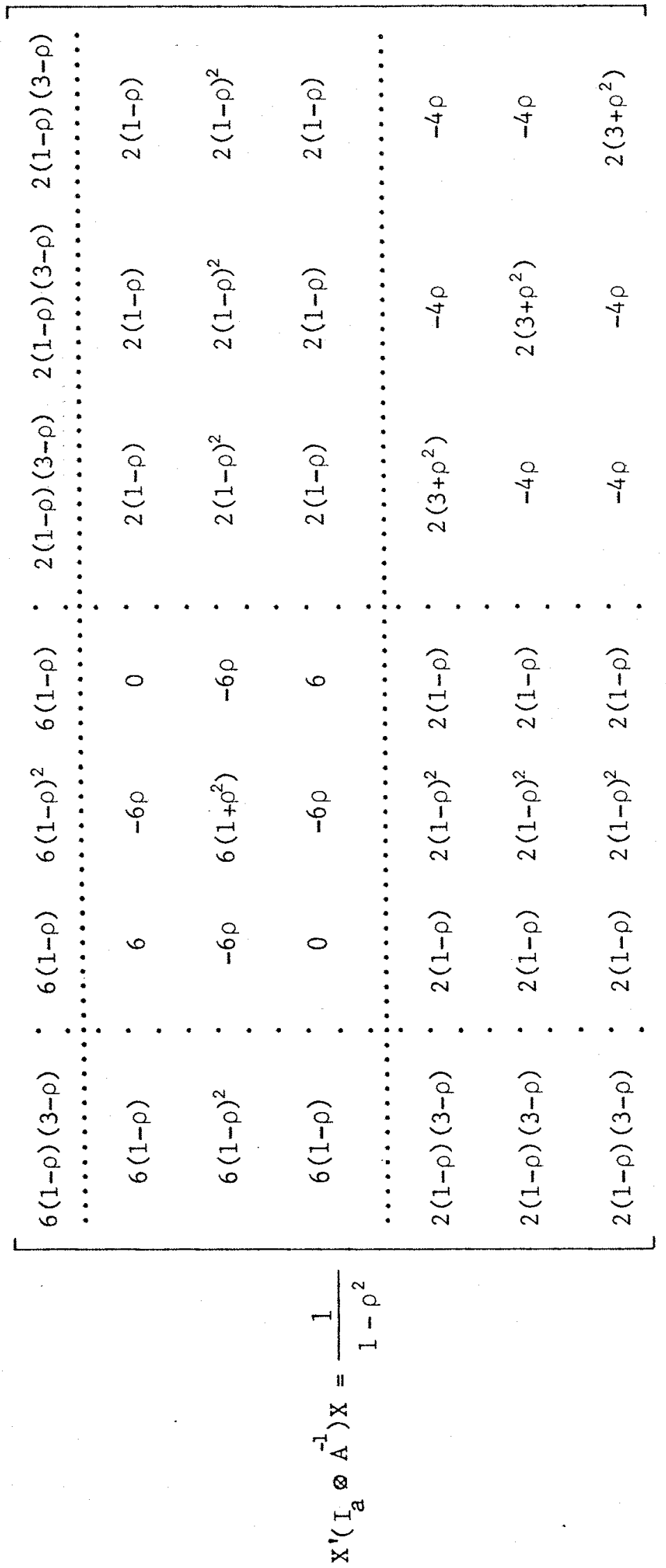


$\left(I_{a} \otimes D^{\prime}\right) \underset{\sim}{Y}=\frac{1}{\sqrt{1-\rho^{2}}}$

$$
\begin{aligned}
& {\left[\sqrt{1-\rho^{2}} y_{111}\right.} \\
& -\rho \mathrm{y}_{111}+\mathrm{y}_{122} \\
& -\rho y_{122}+y_{133} \\
& { }_{\sqrt{1-\rho^{2}}}{ }_{\mathrm{y}_{212}} \ldots \ldots \cdots \cdots \cdots \cdots \\
& -\rho y_{212}+y_{223} \\
& -\rho y_{223}+y_{231} \\
& -\rho y_{423}+y_{432} \\
& -\rho y_{521}+y_{533}
\end{aligned}
$$

$X^{\prime}\left(I_{a} \otimes A^{-1}\right) \underset{\sim}{Y}=\frac{1}{1-\rho^{2}}$

$$
\begin{aligned}
& (1-\rho) \mathrm{P}_{1}+(1-\rho)^{2} \mathrm{P}_{2}+(1-\rho) \mathrm{P}_{3} \\
& \mathrm{P}_{1}-\rho \mathrm{P}_{2} \\
& -\rho \mathrm{P}_{1}+\left(1+\rho^{2}\right) \mathrm{P}_{2}-\rho \mathrm{P}_{3} \\
& -\rho \mathrm{P}_{2}+\mathrm{P}_{3} \\
& \mathrm{~T}_{1}-\rho\left(\mathrm{P}_{11}+\mathrm{P}_{21}+\mathrm{P}_{31}\right)+\rho^{2} \mathrm{~T}_{1}^{(2)} \\
& \mathrm{T}_{2}-\rho\left(\mathrm{P}_{12}+\mathrm{P}_{22}+\mathrm{P}_{32}\right)+\rho^{2} \mathrm{~T}_{2}(2) \\
& \mathrm{T}_{3}-\rho\left(\mathrm{P}_{13}+\mathrm{P}_{23}+\mathrm{P}_{33}\right)+\rho^{2} \mathrm{~T}_{3}^{(2)} \text {. }
\end{aligned}
$$


onde: $\mathrm{p}_{1}=\mathrm{y}_{111}+\mathrm{y}_{212}+\mathrm{y}_{313}+\mathrm{y}_{411}+\mathrm{y}_{512}+\mathrm{y}_{613}$ é o total do 19 periodo; $\mathrm{P}_{2}$ e $\mathrm{P}_{3}$ totais dos periodos II e III, respectivamente;

$$
\begin{aligned}
& \mathrm{T}_{1}=\mathrm{y}_{111}+\mathrm{y}_{231}+\mathrm{y}_{321}+\mathrm{y}_{411}+\mathrm{y}_{521}+\mathrm{y}_{631} \text { é o total do } 19 \text { trata } \\
& \mathrm{T}_{2} \text { e } \mathrm{T}_{3} \text { analogamente definidos; }
\end{aligned}
$$

$\mathrm{T}_{1}^{(2)}=\mathrm{y}_{321}+\mathrm{y}_{521}$ é o total do 19 tratamento no 2 o período;

$\mathrm{T}_{2}^{(2)}$ e $\mathrm{T}_{3}^{(2)}$ analogamente definidos;

$P_{j k}$ é o total das observações que no periodo $j$ precedem ou sucedem o tratamento $\mathrm{k}$, isto $\dot{\mathrm{e}}$ :

$$
\begin{aligned}
& P_{11}=y_{313}+y_{512} \\
& \mathrm{P}_{12}=\mathrm{y}_{111}+\mathrm{y}_{613} \quad \Rightarrow \mathrm{P}_{11}+\mathrm{P}_{12}+\mathrm{P}_{13}=\mathrm{P}_{1} \\
& P_{13}=y_{212}+y_{411} \\
& \mathrm{p}_{21}=\mathrm{y}_{122}+\mathrm{y}_{223}+\mathrm{y}_{423}+\mathrm{y}_{622} \\
& \mathrm{P}_{22}=\mathrm{y}_{223}+\mathrm{y}_{321}+\mathrm{y}_{423}+\mathrm{y}_{521} \quad \Rightarrow \mathrm{P}_{21}+\mathrm{P}_{22}+\mathrm{P}_{23}=2 \mathrm{P}_{2} \\
& \mathrm{P}_{23}=\mathrm{y}_{122}+\mathrm{y}_{321}+\mathrm{y}_{521}+\mathrm{y}_{622} \\
& \mathrm{P}_{31}=\mathrm{y}_{332}+\mathrm{y}_{533} \\
& \mathrm{P}_{32}=\mathrm{y}_{133}+\mathrm{y}_{631} \Rightarrow \mathrm{P}_{31}+\mathrm{P}_{32}+\mathrm{P}_{33}=\mathrm{P}_{3} \\
& \mathrm{P}_{33}=\mathrm{y}_{231}+\mathrm{y}_{432}
\end{aligned}
$$

Nas matrizes $X^{\prime}\left(I_{a} \otimes A^{-1}\right) X$ e $X^{\prime}\left(I_{a} \otimes A^{-1}\right) \underset{\sim}{Y}$ pode-se observar que a soma das linhas referentes a periodos, bem como a soma das linhas relativas a tra tamentos é igual à linha relativa à média. De fato, na matriz $X^{\prime}\left(I_{a} \otimes A^{-1}\right) \underset{\sim}{Y}$ para as linhas relativas a tratamentos, lembrando que:

$$
\sum_{k} T_{k}=\sum_{j} P_{j} ; \sum_{k} T_{k}^{(2)}=P_{2} \text { e } \sum_{k} P_{j k}=\left\{\begin{array}{lll}
P_{j} & \text { para } j=1,3 \\
2 P_{j} & \text { para } j=2
\end{array}\right.
$$


temos: $\mathrm{T}_{1}+\mathrm{T}_{2}+\mathrm{T}_{3}-\rho\left(\mathrm{P}_{11}+\mathrm{P}_{12}+\mathrm{P}_{13}+\mathrm{P}_{21}+\mathrm{P}_{22}+\mathrm{P}_{23}+\mathrm{P}_{31}+\mathrm{P}_{32}+\mathrm{P}_{33}\right)$

$$
\begin{aligned}
+\rho^{2}\left(\mathrm{~T}_{1}^{(2)}+\mathrm{T}_{2}^{(2)}+\mathrm{T}_{3}^{(3)}\right) & =\mathrm{P}_{1}+\mathrm{P}_{2}+\mathrm{P}_{3}-\rho\left(\mathrm{P}_{1}+2 \mathrm{P}_{2}+\mathrm{P}_{3}\right)+\sigma^{2} \mathrm{P}_{2} \\
& =(1-\rho) \mathrm{P}_{1}+(1-\rho)^{2} \mathrm{P}_{2}+(1-\rho) \mathrm{P}_{3},
\end{aligned}
$$

que è a linha referente à média.

No caso geral de $K$ tratamentos e $p$ periodos $\operatorname{com} p \leq K$, te remos

$$
X^{\prime}\left(I_{a} \otimes A^{-1}\right) X=\frac{1}{1-\rho^{2}}\left[\begin{array}{ccc}
c & L^{\prime} & M^{\prime} \\
\sim & P & N^{\prime} \\
\sim & N & T
\end{array}\right]
$$

onde: $c=a(1-\rho)[p-(p-2) \rho]$

$$
\begin{aligned}
& L^{\prime}=a(1-\rho)[1,(1-\rho),(1-\rho), \ldots,(1-\rho), 1]_{(1 \times p)} \\
& \underline{\sim}^{\prime}=\frac{r}{p}(1-\rho)[p-(p-2) \rho]\left[\begin{array}{llll}
1 & 1 & \ldots & 1
\end{array}\right]_{(1 \times K)} \\
& =\frac{r}{p}(1-\rho)[p-(p-2) \rho] I_{\sim}^{\prime}(1 \times K)
\end{aligned}
$$

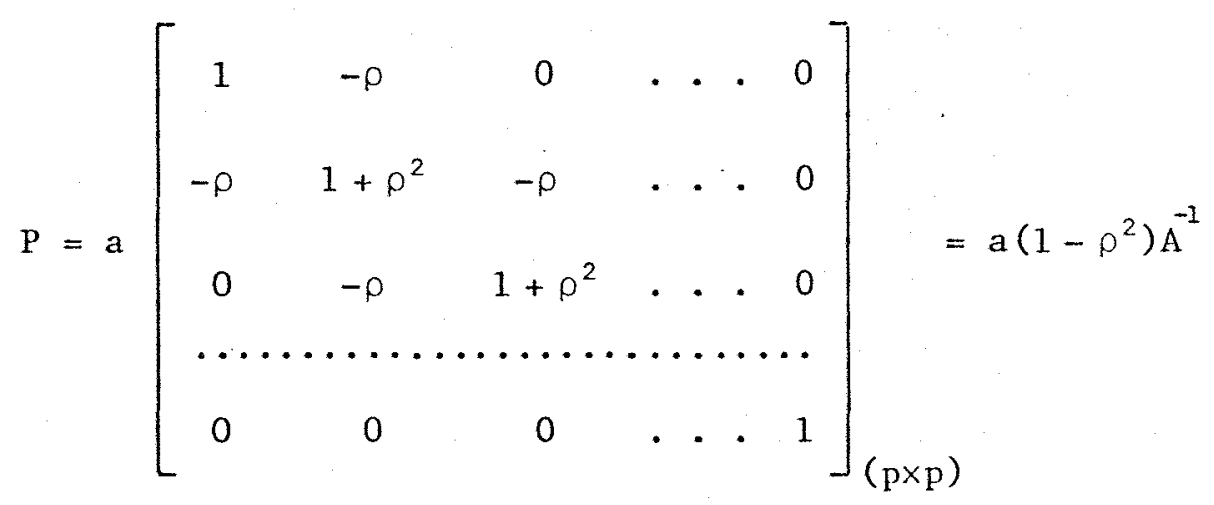




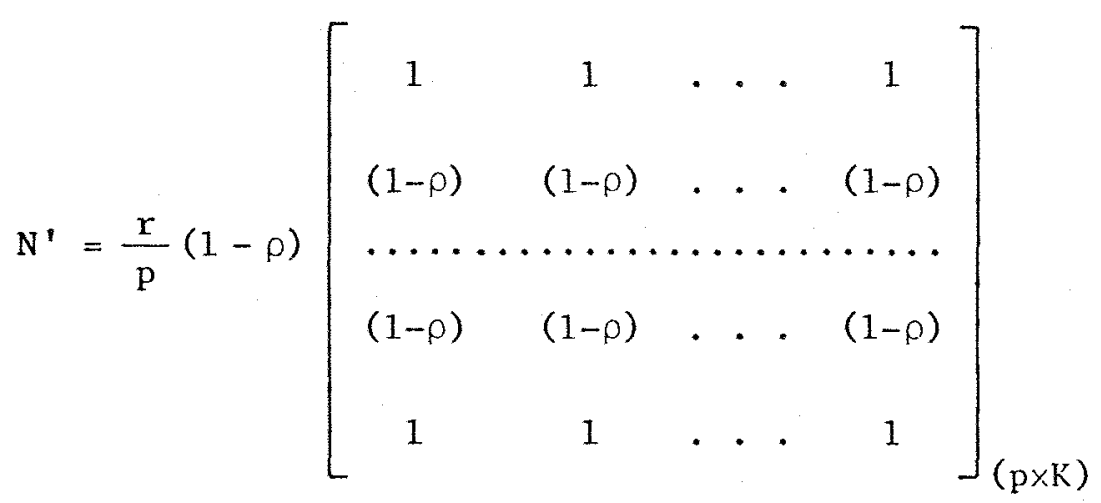

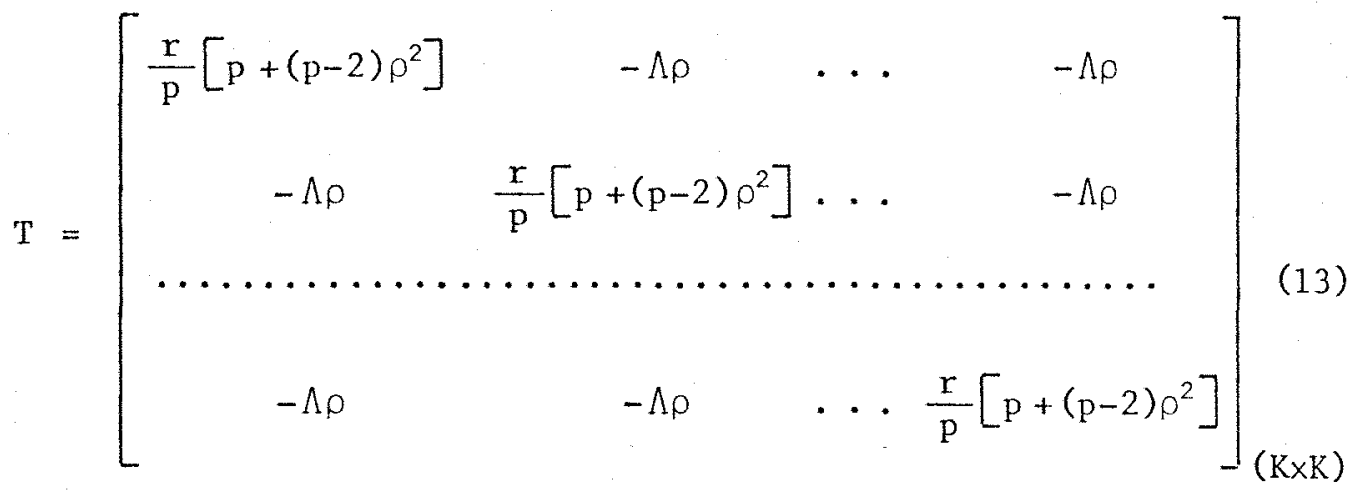

onde $\Lambda$, pela condição de balanceamento vista em 2.1.2, é dado por

$$
\Lambda=\frac{2 a(p-1)}{K(K-1)}
$$

De fato, sendo a soma das linhas referentes a tratamentos igual a linha relativa à media, então, das matrizes $\underset{\sim}{M^{\prime}}$ e $T$, obtemos

$$
\frac{r}{p}\left[p+(p-2) \rho^{2}\right]-(K-1) \Lambda \rho=\frac{r}{p}(1-\rho)[p-(p-2) \rho]
$$

donde se obtëm $\Lambda=\frac{2 r(p-1)}{p(K-1)}$, como era de se esperar, lembrando que $a p=r k$.

Por outro lado, para o caso geral, o segundo membro do sis tema de equações normais será: 


$$
X^{8}\left(I_{a} \otimes A^{-I}\right) Y=\frac{1}{1-\rho^{2}}\left[\begin{array}{c}
G(\rho) \\
\underset{\sim}{P}(\rho) \\
\underset{\sim}{T}(\rho)
\end{array}\right]
$$

onde:

$$
G(\rho)=(1-\rho) P_{1}+(1-\rho)^{2} \sum_{j=2}^{p-1} P_{j}+(1-\rho) P_{p}
$$

$$
\underset{\sim}{P}(\rho)=\left[\begin{array}{c}
\mathrm{P}_{1}-\rho \mathrm{P}_{2} \\
-\rho \mathrm{P}_{1}+\left(1+\rho^{2}\right) \mathrm{P}_{2}-\rho \mathrm{P}_{3} \\
-\rho \mathrm{P}_{2}+\left(1+\rho^{2}\right) \mathrm{P}_{3}-\rho \mathrm{P}_{4} \\
\ldots \ldots \ldots \mathrm{P} \ldots \ldots \\
-\rho \mathrm{P}_{\mathrm{p}-2}+\left(1+\rho^{2}\right) \mathrm{P}_{\mathrm{p}-1}-\rho \mathrm{P}_{\mathrm{p}} \\
-\rho \mathrm{P}_{\mathrm{p}-1}+\mathrm{P}_{\mathrm{p}}
\end{array}\right]_{(\mathrm{p} \times 1)}
$$

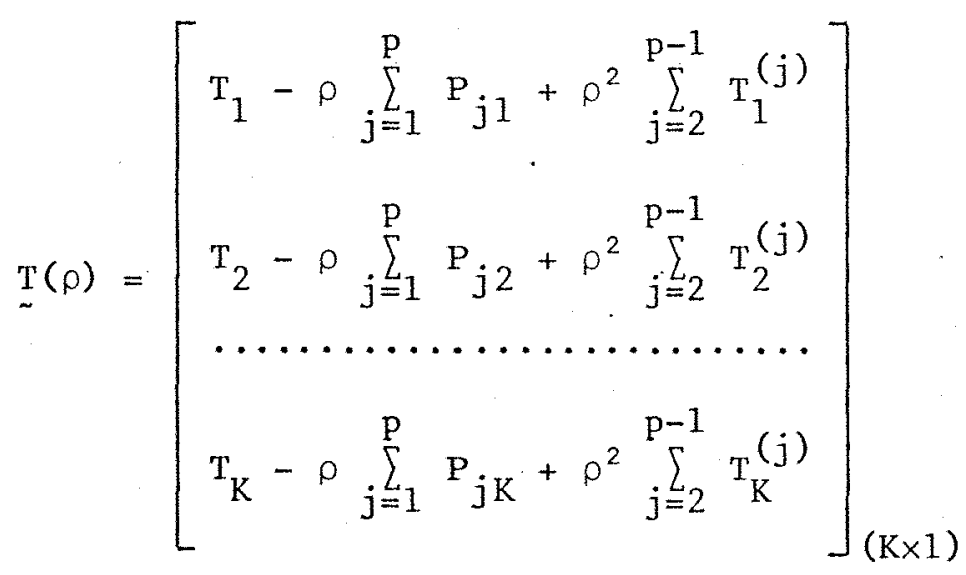

com $\mathrm{P}_{\mathrm{j}}:$ total do periodo $j ; j=1,2, \ldots, p$

$\mathrm{T}_{\mathrm{k}}: \quad$ total do tratamento $\mathrm{k} ; \mathrm{k}=1,2, \ldots, \mathrm{k}$

$P_{j k}$ : soma das observações que no período $j$ precedem ou sucedem o tratamento $\mathrm{k}$

$\mathrm{T}_{\mathrm{k}}^{(\mathrm{j})}$ : total do tratamento $k$ no periodo $j$ 
satisfazendo:

$$
\begin{aligned}
& \sum_{j} P_{j}=\sum_{k} T_{k} ; \sum_{j k} \sum_{j k} P_{k}=P_{1}+2 \sum_{j=2}^{p-1} P_{j}+P_{p} ; \sum_{k} T_{k}^{(j)}=P_{j} \text { (17) } \\
& \text { O sistema de equações normais (7) na forma matricial será }
\end{aligned}
$$

então:

$$
\frac{1}{1-\rho^{2}}\left[\begin{array}{ccc}
c & L^{\prime} & M^{\prime} \\
\underset{\sim}{L} & P & N^{\prime} \\
\underset{\sim}{M} & N & T
\end{array}\right] \underset{\sim}{b}=\frac{1}{1-\rho^{2}}\left[\begin{array}{c}
G(\rho) \\
\underset{\sim}{P}(\rho) \\
\underset{\sim}{T}(\rho)
\end{array}\right]
$$

Se considerarmos a partição

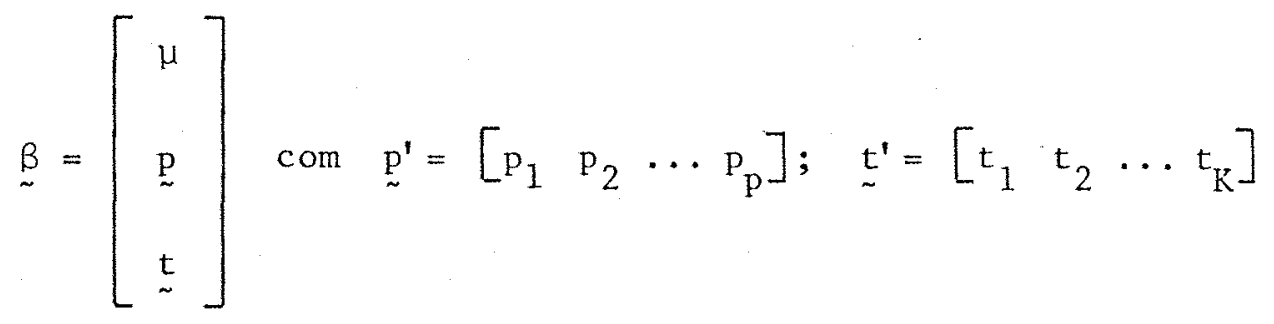

obtemos

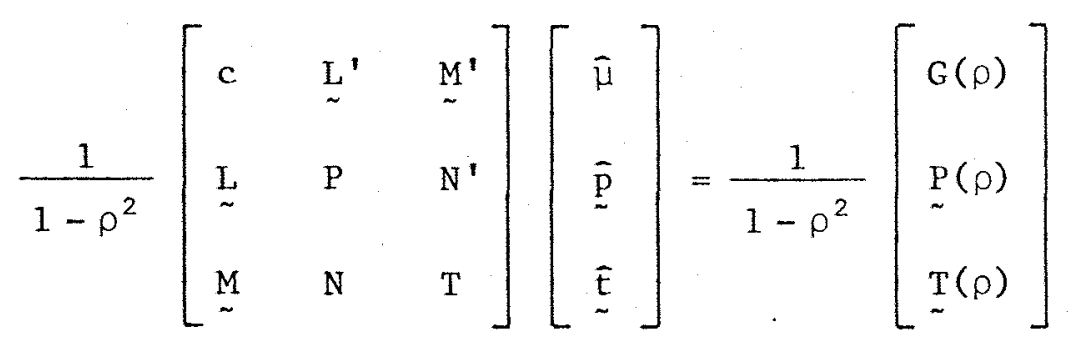

4.1.3. Soma de quadrados de tratamentos ajustada

Pré-multiplicando ambos os membros de (18) pela matriz não singular

$$
\mathrm{W}=\left[\begin{array}{ccc}
1 & \underline{\sim}^{\prime} & 0^{\prime} \\
\underset{\sim}{0} & \mathrm{I}_{\mathrm{p}} & \phi \\
\underset{\sim}{0} & -\mathrm{NP}^{-1} & \mathrm{I}_{\mathrm{K}}
\end{array}\right]
$$


obtemos

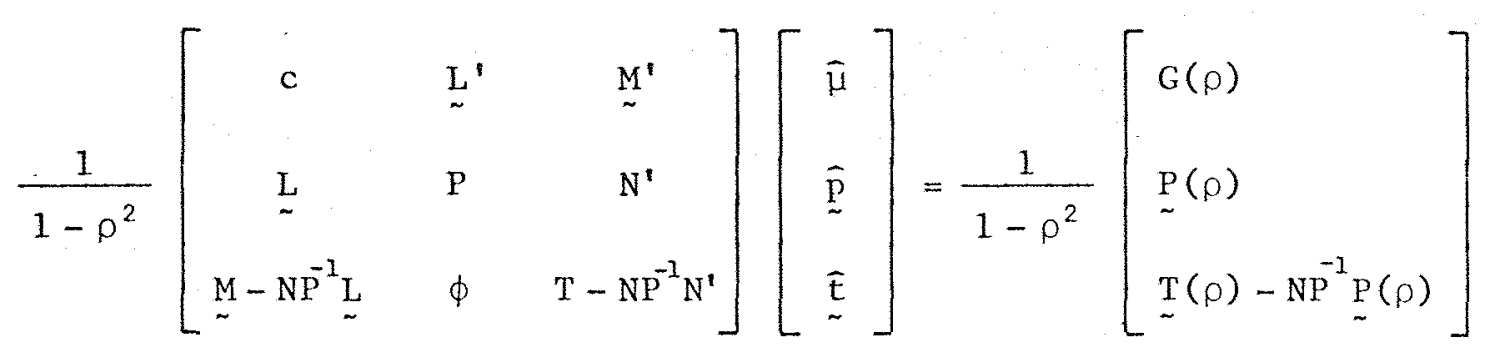

De (11) temos:

$$
P=a\left(1-\rho^{2}\right) A^{-1} \Rightarrow P^{-1}=\frac{1}{a\left(1-\rho^{2}\right)} A
$$

ou

$$
\mathrm{p}^{-1}=\frac{1}{\mathrm{a}\left(1-\rho^{2}\right)}\left[\begin{array}{llll}
1 & \rho & \cdot & \rho^{\mathrm{p}-1} \\
\vdots & 1 & \cdot & \cdot \rho^{\mathrm{p}-2} \\
\rho^{\mathrm{p}-1} & \rho^{\mathrm{p}-2} & \cdots & \vdots \\
& & \cdot & 1
\end{array}\right]
$$

Usando (12) temos:

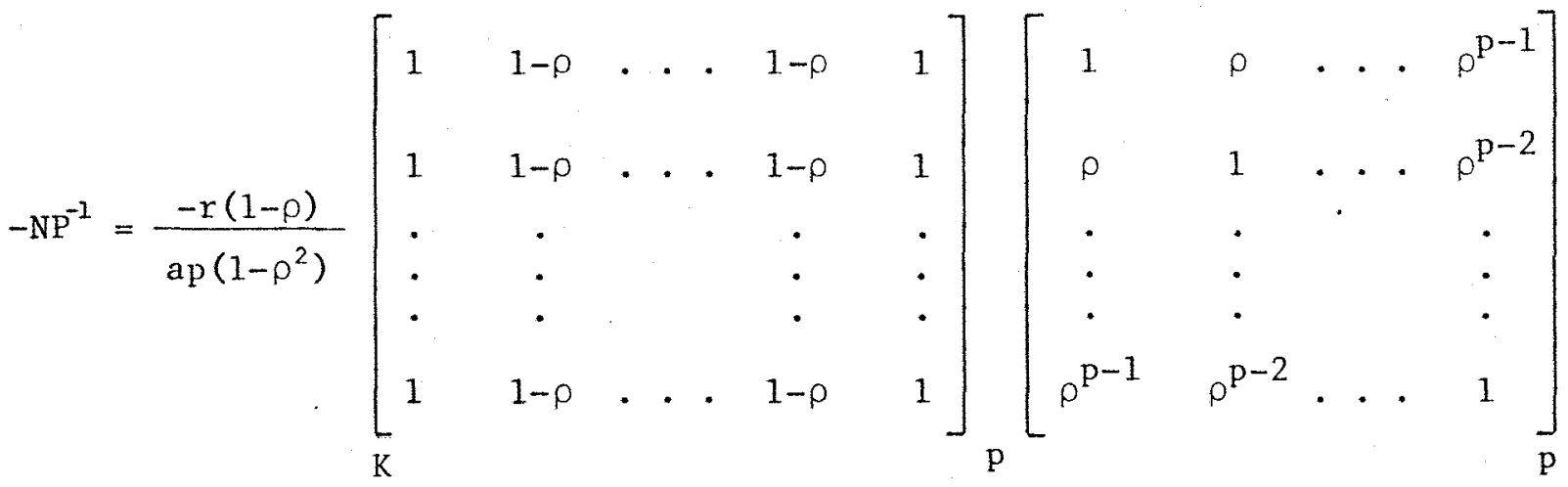

$$
\begin{aligned}
& =\frac{-r(1-\rho)(1+\rho)}{\operatorname{ap}\left(1-\rho^{2}\right)}\left[\begin{array}{cccccc}
1 & 1 & . & \cdot & 1 \\
1 & 1 & . & . & . & 1 \\
\ldots & \ldots & \ldots & \ldots & \ldots \\
1 & 1 & . & . & . & 1
\end{array}\right]_{(\mathrm{K} \times \mathrm{p})}=-\frac{1}{\mathrm{~K}} \mathrm{E}_{(\mathrm{K} \times \mathrm{p})}
\end{aligned}
$$

pois $a p=r K$. 
Usando (9) vem

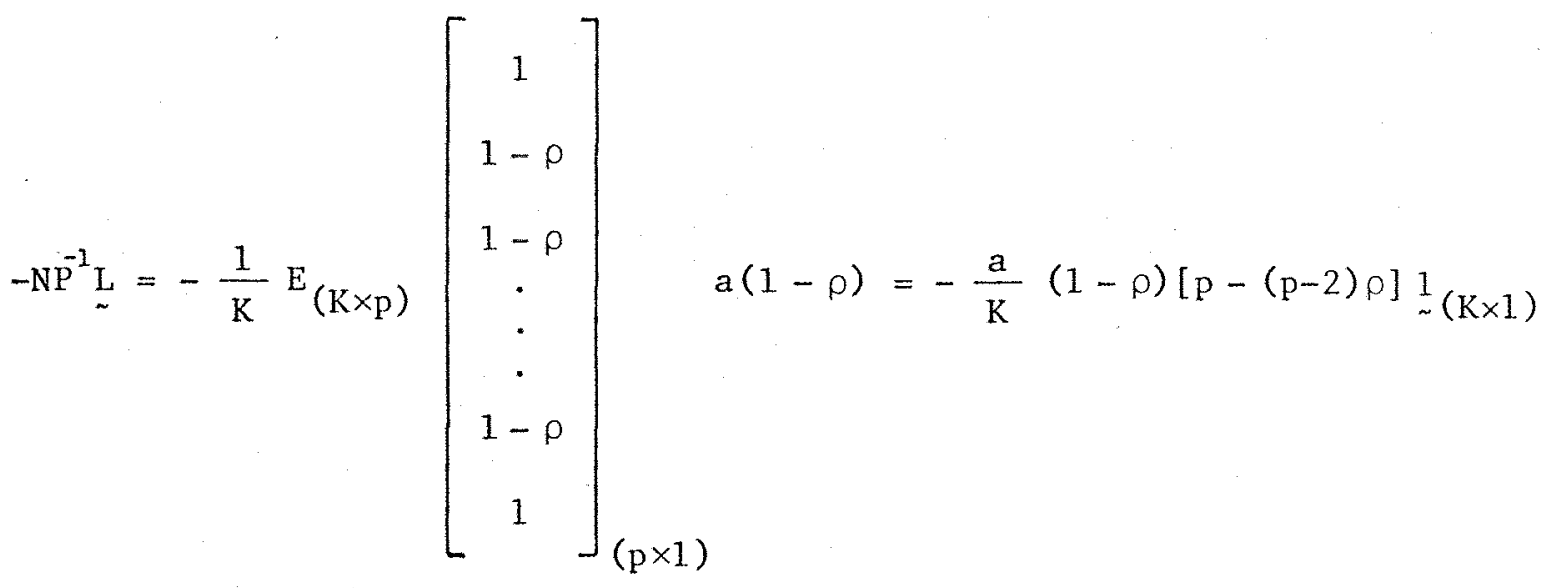

Por $(10)$, lembrando que ap $=r k$, temos que:

$$
\underset{\sim}{M}-N P^{-1} L=\underset{\sim}{O}
$$

Por outro lado

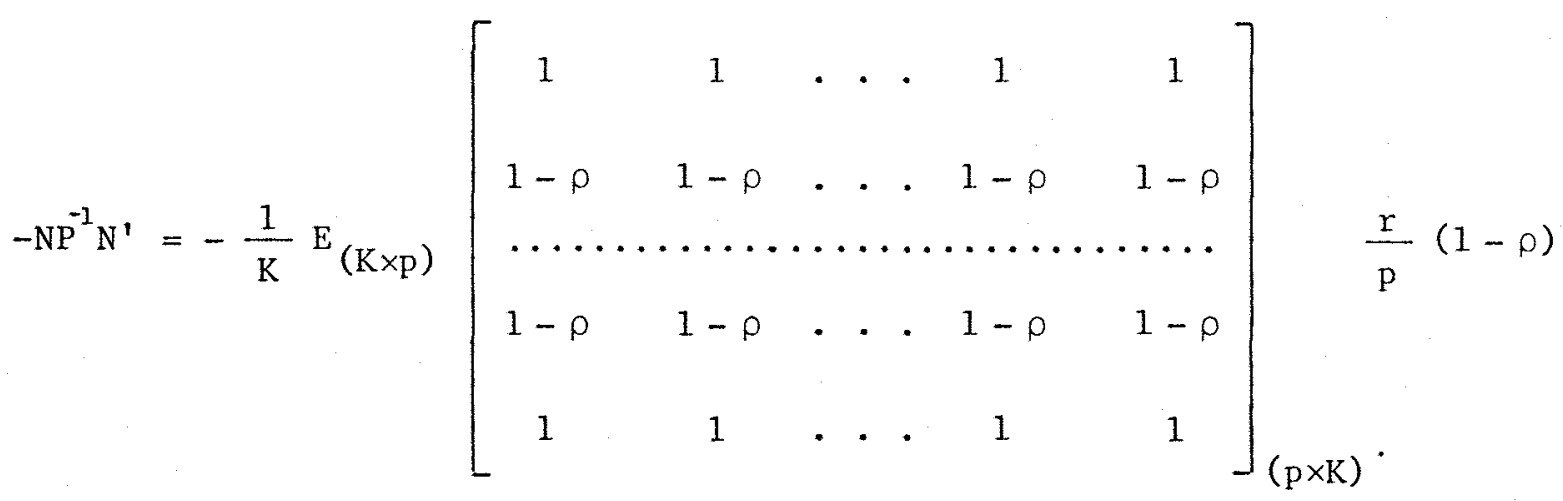

$$
\begin{aligned}
& =-\frac{r}{p K}(1-\rho)[p-(p-2) \rho] E_{(K \times K)}
\end{aligned}
$$

Definindo

$$
\mathrm{C}=\mathrm{T}-\mathrm{NP}^{-1} \mathrm{~N}^{\prime}=\left[\mathrm{C}_{\mathrm{kk}} \cdot\right]_{(\mathrm{K} \times \mathrm{K})}
$$

e usando (13) obtemos

$$
c_{k k}=\frac{r}{p}\left[p+(p-2) \rho^{2}\right]-\frac{r}{p K}(1-\rho)[p-(p-2) \rho]
$$




$$
\mathrm{C}_{\mathrm{kk}}=\left[\frac{\mathrm{r}(\mathrm{p}-2)(\mathrm{K}-1)}{\mathrm{pK}}\right] \rho^{2}+\left[\frac{2 \mathrm{r}(\mathrm{p}-1)}{\mathrm{pK}}\right] \rho+\frac{(\mathrm{K}-1) \mathrm{r}}{\mathrm{K}}
$$

e

$$
\begin{aligned}
\mathrm{C}_{k^{\prime}} & =\frac{-2 r(p-1)}{p(K-1)} \rho-\frac{r}{p K}(1-\rho)[p-(p-2) \rho] \\
& =-\left[\frac{r(p-2)}{p K}\right] \rho^{2}-\left[\frac{2 r(p-1)}{p K(K-1)}\right] \rho-\frac{r}{k} ; \text { para } k \neq k^{\prime}
\end{aligned}
$$

ou seja

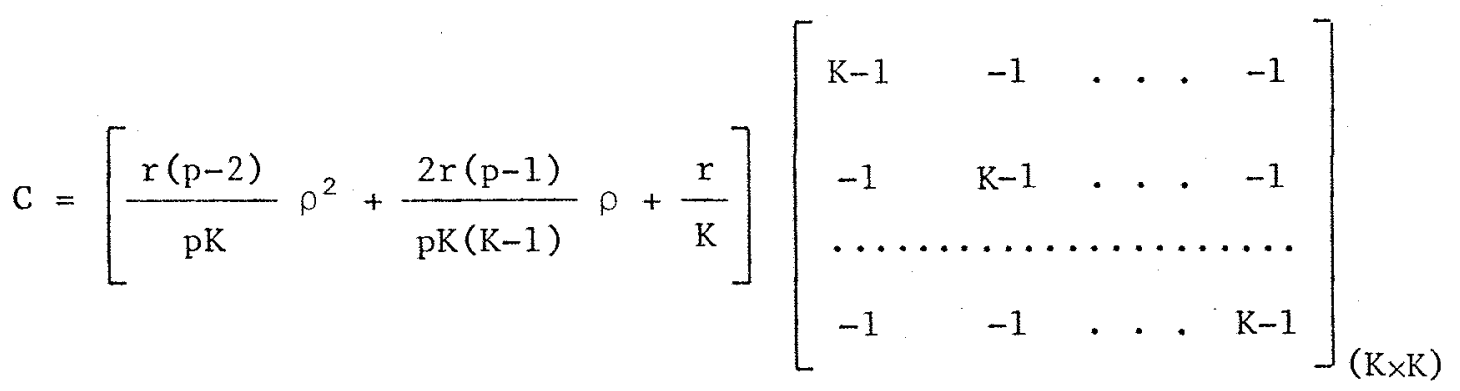

donde se percebe que posto $(\mathrm{C})=\mathrm{K}-1$.

Para o segundo membro do sistema (18), definindo

$$
\underset{\sim}{Q}(\rho)=\underset{\sim}{T}(\rho)-N P^{-1} \underset{\sim}{P}(\rho)
$$

então de (15) e (21) temos:

$$
-N P_{\sim}^{-1} \underset{\sim}{P}(\rho)=-\frac{1}{K} E_{(K \times p)}\left[\begin{array}{c}
P_{1}-\rho P_{2} \\
-\rho P_{1}+\left(1+\rho^{2}\right) P_{2}-\rho P_{3} \\
\ldots \ldots \ldots \ldots \ldots \ldots \ldots \ldots \\
-\rho P_{p-2}+\left(1+\rho^{2}\right) P_{p-1}-\rho P_{p} \\
-\rho P_{p-1}+P_{p}
\end{array}\right]_{(p \times 1)}
$$

com $G(\rho)$ definido em (14). 


$$
\begin{aligned}
& \text { Portanto, } \underset{\sim}{Q}(\rho)=\left[Q_{k}(\rho)\right]_{(K \times 1)} \text { com } \\
& Q_{k}(\rho)=T_{k}(\rho)-\frac{1}{K} G(\rho)
\end{aligned}
$$

onde $T_{k}(\rho)$ é definido em (16).

Observamos que:

$$
\begin{aligned}
\sum_{k=1}^{K} Q_{k}(\rho) & =\sum_{k=1}^{K} T_{k}(\rho)-\frac{1}{K} \sum_{k=1}^{K} G(\rho) \\
& =\sum_{k=1}^{K} T_{k}(\rho)-G(\rho)
\end{aligned}
$$

De (16)

$$
\sum_{k=1}^{K} T_{k}(\rho)=\sum_{k} T_{k}-\rho \sum_{k} \sum_{j} P_{j k}+\rho^{2} \sum_{k} \sum_{j=2}^{p-1} T_{k}^{(j)}
$$

Das igualdades em (17) vem:

$$
\begin{aligned}
\sum_{k=1}^{K} T_{k}(\rho) & =\sum_{j} P_{j}-\rho\left(P_{1}+2 \sum_{j=2}^{p-1} P_{j}+P_{p}\right)+\rho^{2} \sum_{j=2}^{p-1} P_{j} \\
& =(1-\rho) P_{1}+(1-\rho)^{2} \sum_{j=2}^{p-1} P_{j}+(1-\rho) P_{p}=G(\rho)
\end{aligned}
$$

Levando em (27) obtemos

$$
\sum_{k=1}^{K} Q_{k}(\rho)=0
$$

Substituindo (22), (24) e (26) em (19) obtemos o sistema de equações :

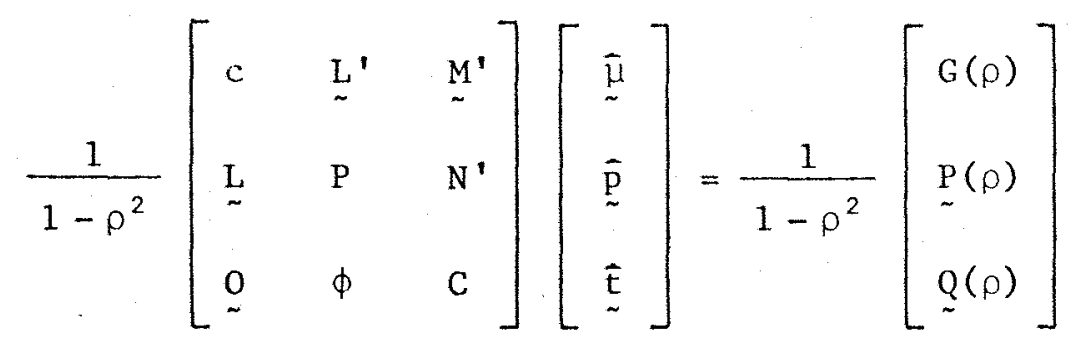


De (29) podemos obter a equação normal para tratamentos ajustada para média e período, ou seja

$$
\frac{1}{1-\rho^{2}} C \underset{\sim}{\hat{t}}=\frac{1}{1-\rho^{2}} \stackrel{Q}{Q}(\rho)
$$

De $(24)$ e (28) temos que posto $(C)=K-1$ e $\sum_{k} Q_{k}(\rho)=0$, portanto, utili zando a restrição $\sum_{k=1}^{K} t_{k}=0$, a qual implica em $\sum_{k=1}^{K} \hat{t}_{k}=0$, podemos obter a solução de (30) resolvendo o sistema:

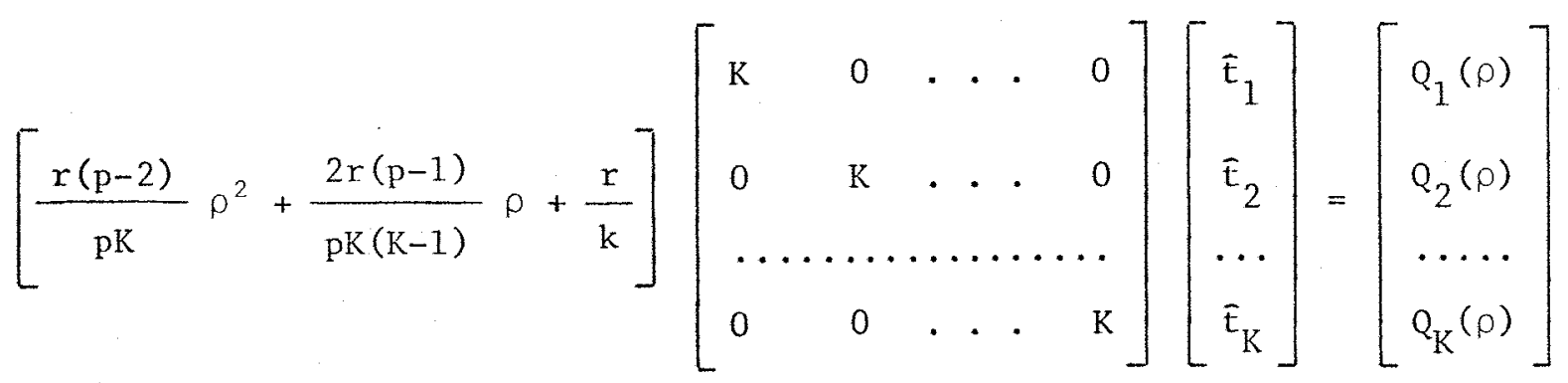
ou

$$
\left[\frac{r(p-2)}{p} \rho^{2}+\frac{2 r(p-1)}{p(K-1)} \rho+r\right] I_{K} \bar{t}=\underset{\sim}{Q}(\rho)
$$

Lembrando que ap $=r K$ e $\Lambda=\frac{2 r(p-1)}{p(K-1)}$ obtemos

$$
\bar{t}_{k}=\frac{1}{\frac{a(p-2)}{k} \rho^{2}+\Lambda \rho+r} Q_{k}(\rho)
$$

e utilizando (30) vem

$S Q\{$ Tratamentos aj. $\}=\sum_{k=1}^{K} E_{k} Q_{k}(\rho) \frac{1}{1-\rho^{2}}$

ou

$S Q\{\operatorname{Tratamentos}$ a j. $\}=\frac{1}{\left[\frac{\mathrm{a}(\mathrm{p}-2)}{\mathrm{K}} \rho^{2}+\Lambda \rho+\mathrm{r}\right]\left(1-\rho^{2}\right)} \sum_{\mathrm{k}=1}^{\mathrm{K}} \mathrm{Q}_{\mathrm{k}}^{2}(\rho)$ 
4.1.4. Somas de quadrados de periodos e média ignoran do tratamentos

Se no modelo linear $\underset{\sim}{Y}=\underset{\sim}{X} \underset{\sim}{\beta}+\underset{\sim}{e}$ ignorarmos efeitos de trata mentos, então do sistema (18) as equações normais na forma matricial para periodos e média serão:

$$
\frac{1}{1-\rho^{2}}(L \bar{\mu}+P \hat{p})=\frac{1}{1-\rho^{2}} \underset{\sim}{P}(\rho)
$$

fazendo $\hat{\mu}=0$ podemos obter uma solução do sistema

$$
\frac{1}{1-\rho^{2}} P \underset{\sim}{p}=\frac{1}{1-\rho^{2}} \underset{\sim}{P}(\rho)
$$

ou, por (11)

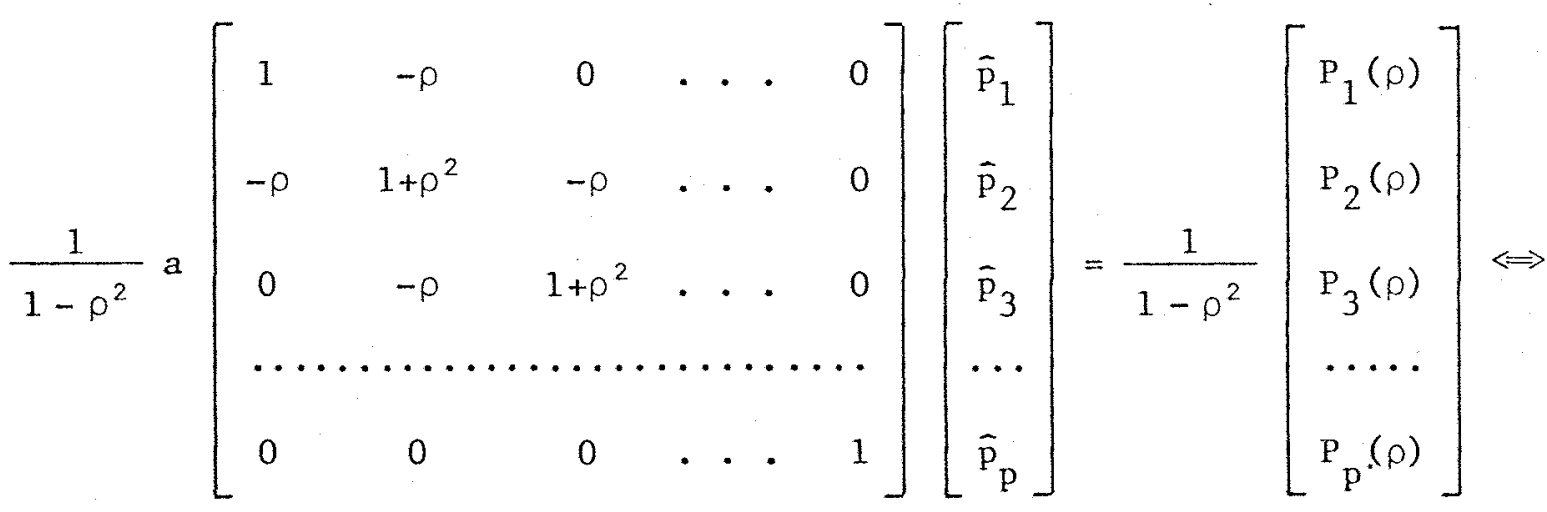

$\Leftrightarrow \frac{1}{1-\rho^{2}} \mathrm{a}\left(1-\rho^{2}\right) \mathrm{A}^{-1} \underset{\sim}{\mathrm{p}}=\frac{1}{1-\rho^{2}} \underset{\sim}{\mathrm{P}}(\rho) \Rightarrow \underset{\sim}{\hat{\mathrm{p}}}=\frac{1}{\mathrm{a}\left(1-\rho^{2}\right)} \mathrm{A} \underset{\sim}{\mathrm{P}}(\rho)$

Usando (33) vem

$$
\mathrm{SQ}\{\text { Períodos e Média }\}=\frac{1}{a\left(1-\rho^{2}\right)^{2}} \underline{\sim}^{\prime}(\rho) \mathrm{A} \underset{\sim}{\mathrm{P}}(\rho)
$$

Mas de (15) temos 


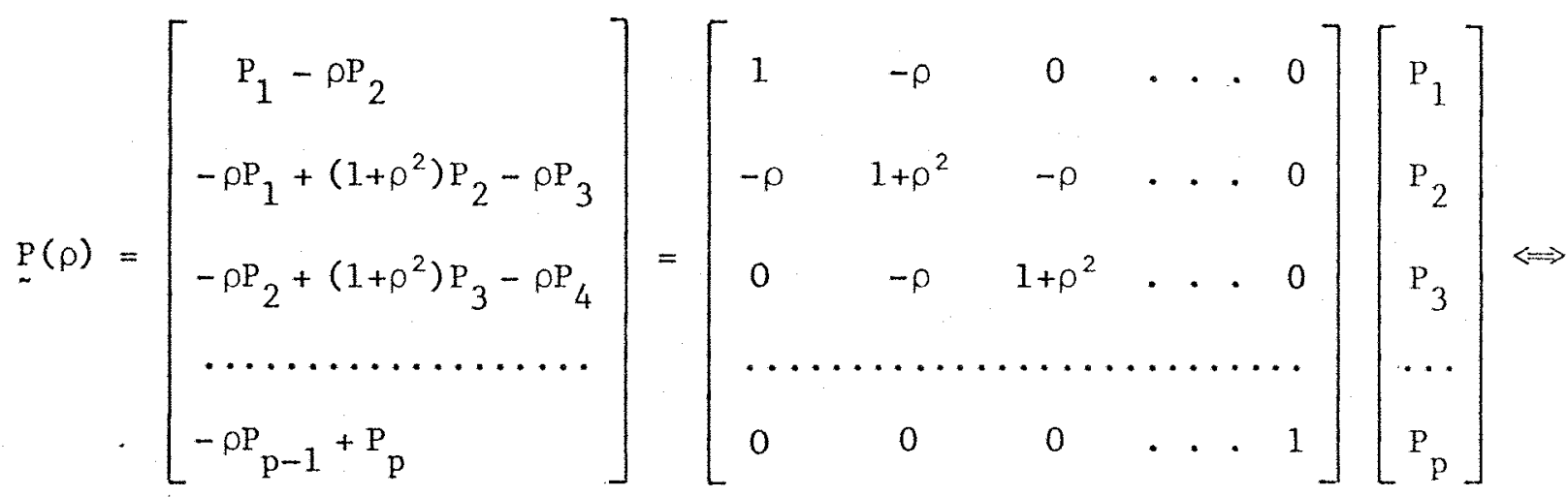

$\Leftrightarrow \underset{\sim}{P}(\rho)=\left(1-\rho^{2}\right) A^{-1} \underset{\sim}{P}$

Levando em (34) obtemos

$$
\underset{\sim}{\hat{p}}=\frac{1}{a} \underset{\sim}{P} \quad \therefore \quad \hat{\mathrm{p}}_{j}=\frac{1}{a} \mathrm{P}_{j}
$$

E (35) pode ser escrita

$$
\text { SQ }\{\text { Periodos e Média }\}=\frac{1}{a\left(1-\rho^{2}\right)} \sum_{j=1}^{p} P_{j} P_{j}(\rho)
$$

Da mesma forma, se no modelo linear geral ignorarmos trata mentos e efeitos de periodos, então do sistema (18) obtemos a equação nor mal para a média

$$
\frac{1}{1-\rho^{2}} \text { с } \bar{\mu}=\frac{1}{1-\rho^{2}} G(\rho)
$$

ou por (8)

$\frac{1}{1-\rho^{2}} a(1-\rho)[p-(p-2) \rho] \bar{\mu}=\frac{1}{1-\rho^{2}} G(\rho) \Rightarrow \bar{\mu}=\frac{G(\rho)}{a(1-\rho)[p-(p-2) \rho]}$

Logo, por (37)

$$
\mathrm{SQ}\{\text { Média }\}=\frac{\mathrm{G}^{2}(\rho)}{\mathrm{a}(1-\rho)\left(1-\rho^{2}\right)[\mathrm{p}-(\mathrm{p}-2) \rho]}
$$


De (36) e (38) obtemos por diferença a Soma de Quadrados de Periodos ajustada para a Média e ignorando tratamentos, ou seja

$S Q\{$ Períodos $\}=S Q\{$ Períodos e Mëdia $\}-S Q\{$ Média $\}$

4.1.5. Soma de quadrados total

Da expressão (5) obtemos

$$
\begin{aligned}
\mathrm{SQ}\{\text { Tota1 }\} & =\underline{\sim}^{\prime}(\rho) \underset{\sim}{Y}(\rho) \\
& =\underset{\sim}{Y^{\prime}}\left(I_{a} \otimes D^{\prime}\right)^{\prime}\left(I_{a} \otimes D^{\prime}\right) \underset{\sim}{Y}
\end{aligned}
$$

ou

$$
\mathrm{SQ}\{\operatorname{Tota} 1\}=\underset{\sim}{Y^{\prime}}\left(I_{\mathrm{a}} \otimes \mathrm{A}^{-1}\right) \underset{\sim}{Y}
$$

onde $\mathrm{Y}_{\sim} \dot{\mathrm{e}}$ o vetor de observações originais.

Desenvolvendo (39) obtemos

$$
S Q\{\text { Tota } 1\}=\frac{1}{1-\rho^{2}} \sum_{i=1}^{a}\left\{\left(1-\rho^{2}\right) y_{i 1}^{2}+\sum_{j=2}^{p}\left(y_{i j}-\rho y_{i(j-1)}\right)^{2}\right\}
$$

Os resultados obtidos são sumarizados no quadro que se se gue:

$$
\text { Anālise da variância no modèlo auto-regressivo }
$$

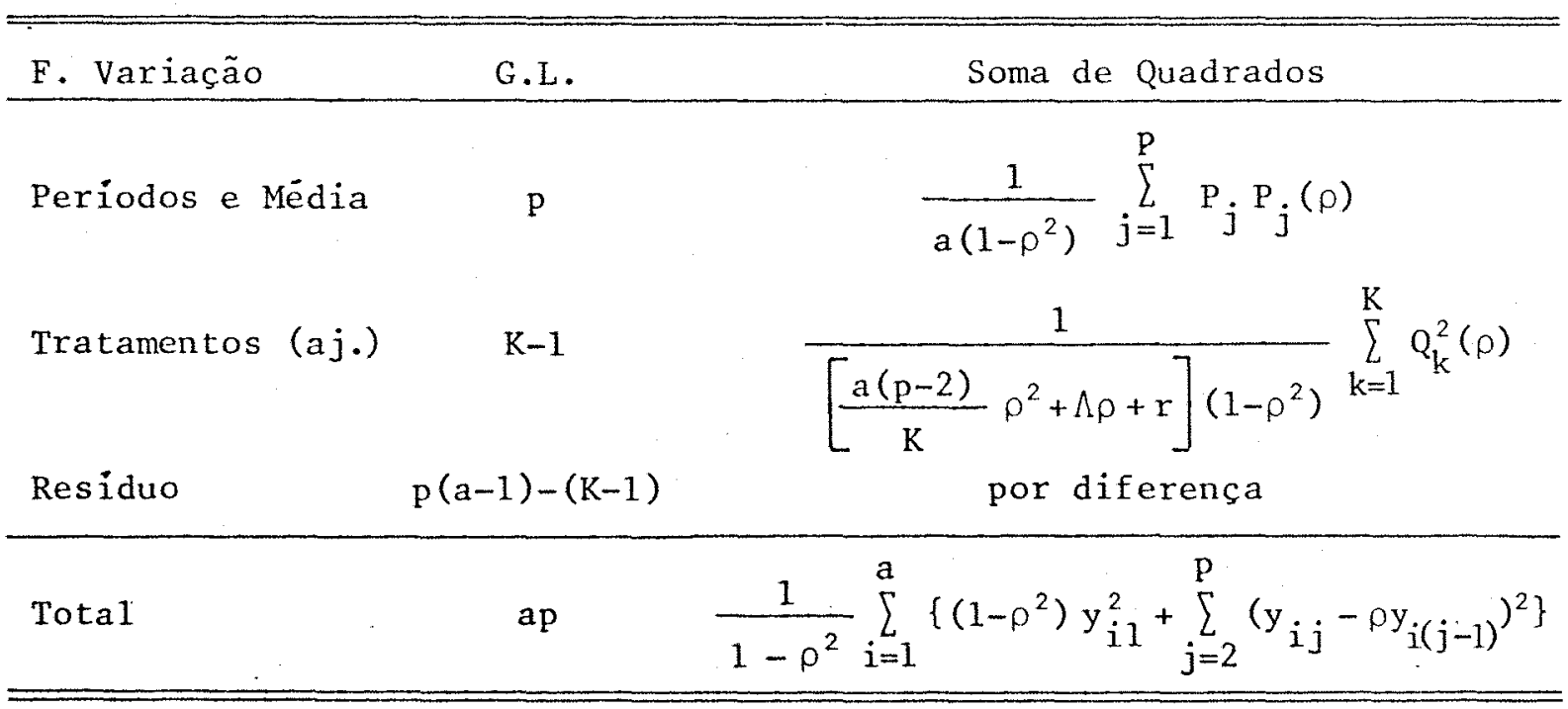


4.1.6. Esperança matemātica da soma de quadrados de tratamentos ajustada

Consideremos a partição

$$
\left(I_{a} \otimes D^{\prime}\right) X=\left[j: x_{1}: x_{2}\right] \frac{1}{\sqrt{1-\rho^{2}}}
$$

$\operatorname{com} \underset{\sim}{j}$ de ordem $(a p \times 1)$ o vetor relativo à média

$\mathrm{x}_{1}$ de ordem (ap $\left.\times p\right)$ a matriz relativa a periodos

$\mathrm{x}_{2}$ de ordem $(a \mathrm{p} \times \mathrm{K})$ a matriz relativa a tratamentos

então o primeiro e segundo membros do sistema (18) podem ser escritos na forma:

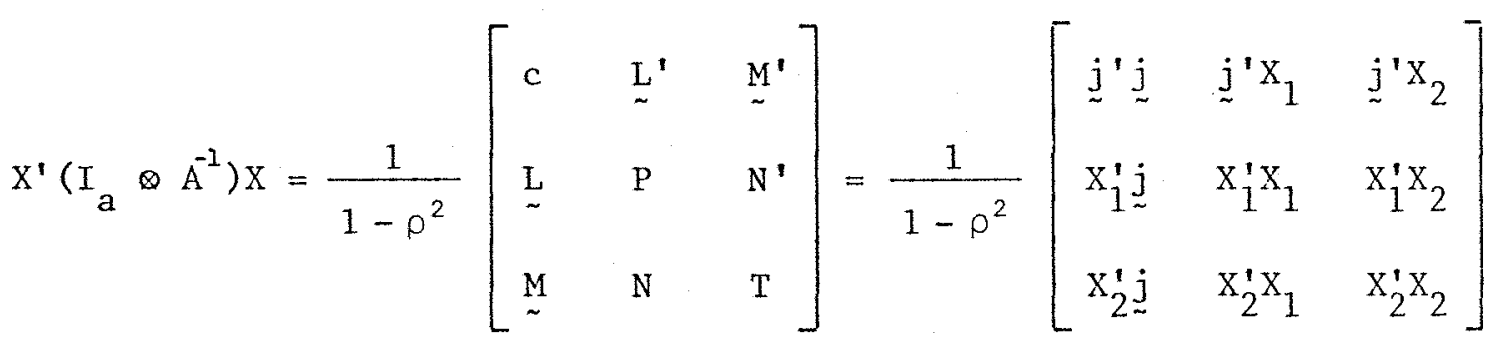

$$
X^{\prime}\left(I_{a} \otimes A^{-I}\right) \underset{\sim}{Y}=X^{\prime}\left(I_{a} \otimes D\right)\left(I_{a} \otimes D^{\prime}\right) \underset{\sim}{Y}=X^{\prime}\left(I_{a} \otimes D\right) \underset{\sim}{\underset{\gamma}{Y}}(\rho)
$$

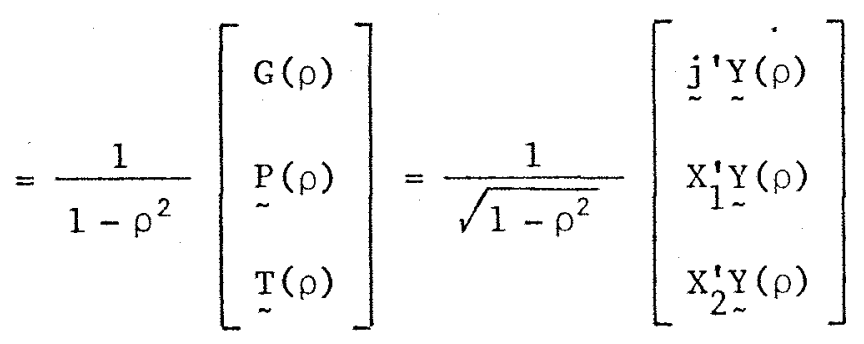

De (25) temos que

$$
\underset{\sim}{Q}(\rho)=\underset{\sim}{T}(\rho)-N P^{-1} P(\rho)
$$

usando (42)

$$
\underset{\sim}{Q}(\rho)=\left[X_{2}^{\prime} \underset{\sim}{Y}(\rho)-N P^{-1} X_{1}^{\prime} \underset{\sim}{Y}(\rho)\right] \sqrt{1-\rho^{2}}
$$




$$
\underset{\sim}{Q}(\rho)=\left(X_{2}^{\prime}-N P^{-1} X_{1}^{\prime}\right) \underset{\sim}{Y}(\rho) \sqrt{1-\rho^{2}}
$$

ou

$$
\underline{Q}(\rho)=H^{\prime} \underset{\sim}{Y}(\rho) \sqrt{1-\rho^{2}}
$$

onde $H^{\prime}=\mathrm{X}_{2}^{\prime}-\mathrm{NP}^{-1} \mathrm{X}_{1}^{\prime}$.

Entretanto, (32) pode ser escrita na forma

$$
S Q\{\text { Tratamentos aj. }\}=\frac{1}{1-\rho^{2}} \stackrel{E^{\prime}}{\sim} Q(\rho)
$$

De (30) temos

$$
C \underset{\sim}{\hat{\mathbf{t}}}=\underset{\sim}{Q}(\rho) \Rightarrow \underset{\sim}{\hat{\mathrm{E}}}=\mathrm{C}^{-} \underset{\sim}{Q}(\rho)
$$

com $\mathrm{C}^{-}$uma inversa generalizada de $\mathrm{C}$

substituindo (45) em (44) vem

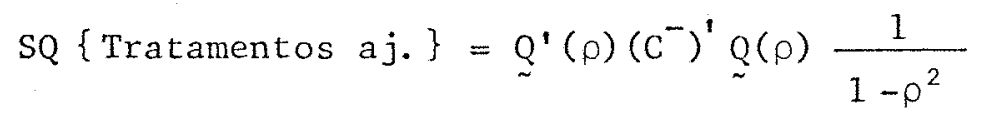

Usando (43) temos

$S Q\{$ Tratamentos aj. $\}=\underset{\sim}{Y^{\prime}}(\rho)\left(X_{2}-X_{1} P^{-1} N^{\prime} \cdot\right)\left(C^{-}\right)^{\prime}\left(X_{2}^{\prime}-N P^{-1} X_{1}^{\prime}\right) \underset{\sim}{Y}(\rho)$

observemos que

$H^{\prime} H=\left(X_{2}^{\prime}-N P^{-1} X_{1}^{\prime}\right)\left(X_{2}-X_{1} P^{-1} N^{\prime}\right)=X_{2}^{\prime} X_{2}-X_{2}^{\prime} X_{1} P^{-1} N^{\prime}-N P^{-1} X_{1}^{\prime} X_{2}+N P^{-1} X_{1}^{\prime} X_{1} P^{-1} N^{\prime}$ que na notação de (41) resulta

$$
H^{\prime} H=T-N P^{-1} N^{\prime}-N P^{-1} N^{\prime}+N P^{-1} P P^{-1} N^{\prime}=T-N P^{-1} N^{\prime}=C
$$

substituindo em (46) vem

$$
\mathrm{SQ}\{\text { Tratamentos aj. }\}=\underset{\sim}{Y^{\prime}}(\rho) H\left[\left(\mathrm{H}^{\prime} H\right)^{-}\right]^{\prime} \mathrm{H}^{\prime} \underset{\sim}{\mathrm{Y}}(\rho)
$$

ou equivalentemente 


$$
S Q\{\text { Tratamentos aj. }\}=S Q\left\{H^{\prime} \underset{\sim}{Y}(\rho)\right\}
$$

Utilizando um resultado devido a SCHEFFE (1959), em

\section{temos}

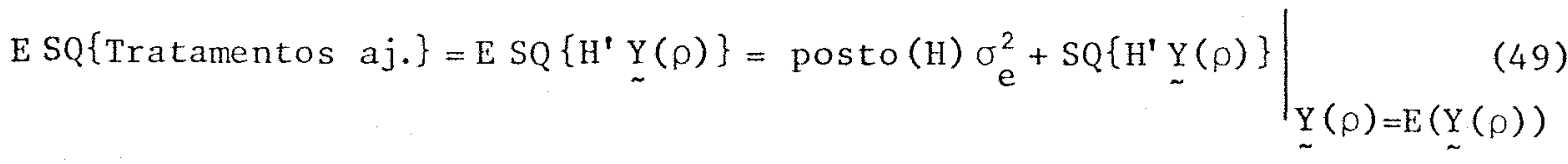

Mas posto $(H)=$ posto $\left(H^{\prime} H\right)=$ posto $(C)=K-1$, por $(47)$ e $(24)$.

Sendo $E \underset{\sim}{(Y}(\rho))=\left(I_{a} \otimes D^{\prime}\right) X \underset{\sim}{\beta}$, temos que

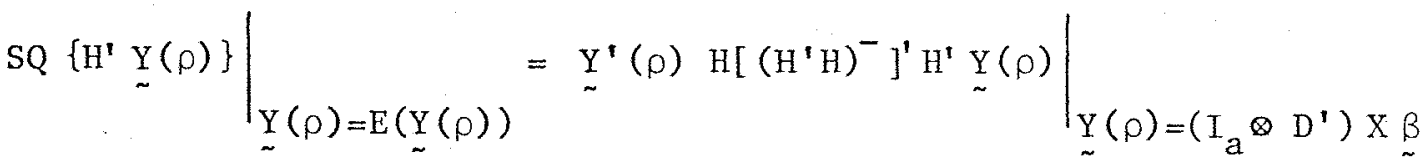

$$
\begin{aligned}
& =\underset{\sim}{\beta^{\prime}} X^{\prime}\left(I_{a} \otimes D\right) H\left[\left(H^{\prime} H\right)^{-}\right]^{\prime} H^{\prime}\left(I_{a} \otimes D^{\prime}\right) X \underset{\sim}{\beta}
\end{aligned}
$$

mas por (40)

$$
\left(I_{a} \otimes D^{\prime}\right) X \underset{\sim}{\beta}=\frac{1}{\sqrt{1-\rho^{2}}}\left[\underset{\sim}{j}: x_{1} \vdots x_{2}\right]\left[\begin{array}{c}
\mu \\
\underset{\sim}{p} \\
t
\end{array}\right]=\frac{1}{\sqrt{1-\rho^{2}}}\left[\begin{array}{c}
\underset{\sim}{j} \mu \\
x_{1}^{p} \\
\mathrm{x}_{2}^{\mathrm{t}}
\end{array}\right]
$$

e

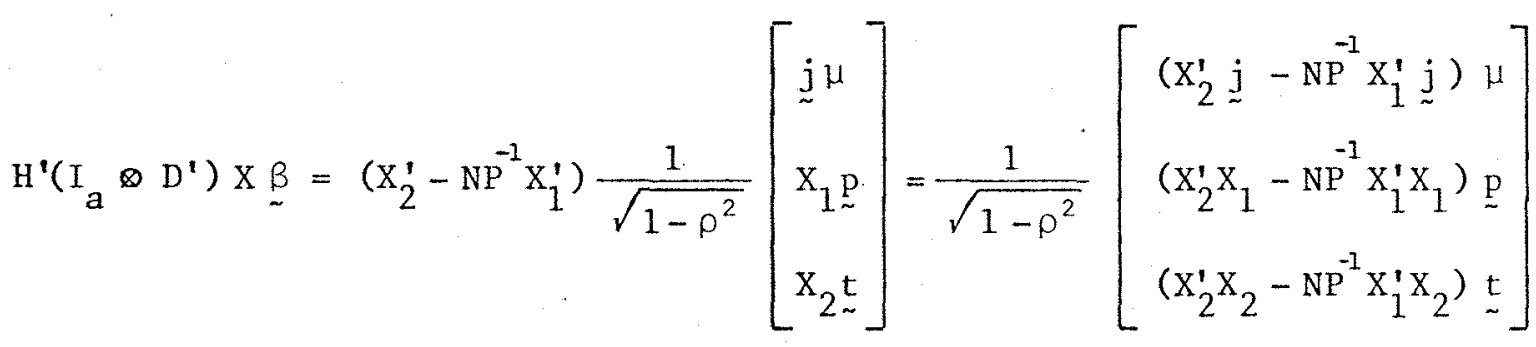

que a notação de (41) fica

$$
H^{\prime}\left(I_{a} \otimes D^{\prime}\right) X \underset{\sim}{\beta}=\frac{1}{\sqrt{1-\rho^{2}}}\left[\begin{array}{c}
\left(\underset{\sim}{(}-N P^{-1} \underline{\sim}\right) \mu \\
\left(N-N P^{-1} P\right) \underset{\sim}{p} \\
\left(T-N P^{-1} N^{\prime}\right) t
\end{array}\right]
$$


Usando (22) e (23) vem

$$
H^{\prime}\left(I_{a} \otimes D^{\prime}\right) \times \underset{\sim}{\beta}=\frac{1}{\sqrt{1-\rho^{2}}}\left[\begin{array}{c}
0 \\
\sim \\
\phi \\
c t \\
\\
\end{array}\right]
$$

substituindo (51) em (50) obtemos

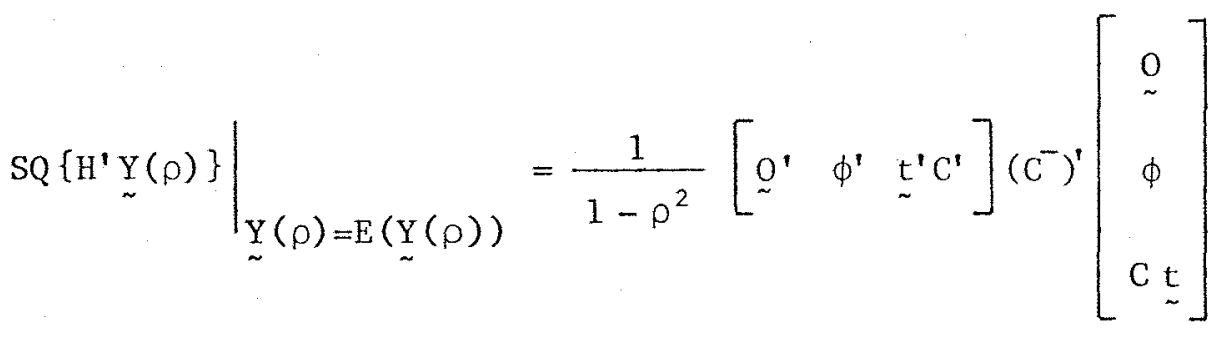

$$
\begin{aligned}
& =\frac{1}{1-\rho^{2}} \stackrel{t^{\prime}}{\mathrm{C}} \underset{\sim}{t}
\end{aligned}
$$

Levando (52) em (49) obtemos

$$
\text { E SQ }\{\text { Tratamentos } a j .\}=(K-1) \sigma_{e}^{2}+\frac{1}{1-\rho^{2}} \underset{\sim}{t} \underset{\sim}{t}
$$

para $c$ definida em (24) e para $\sum_{k=1}^{K} t_{k}=0$ temos desenvolvendo (53), que E SQ $\{$ Tratamentos $a j \cdot\}=(K-1) \sigma_{e}^{2}+\frac{\frac{a(p-2)}{k} \rho^{2}+\Lambda \rho+r}{1-\rho^{2}} \sum_{k=1}^{K} t_{k}^{2}$

4.1.7. Esperança matemática da soma de quadrados de períodos e média, ignorando tratamentos

De (35) temos

$\operatorname{SQ}\{$ Períodos e Médias $\}=\frac{1}{\mathrm{a}\left(1-\rho^{2}\right)^{2}} \underset{\sim}{P^{\prime}}(\rho)$ A $\underset{\sim}{\mathrm{P}}(\rho)$

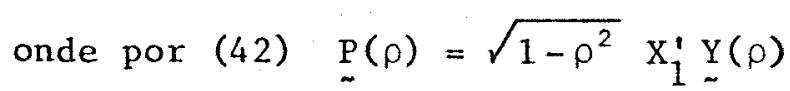


Logo

$$
\text { SQ }\{\text { Periodos e Média }\}=\frac{1}{a\left(1-\rho^{2}\right)} \underset{\sim}{Y^{\prime}(\rho) X_{1}} A_{X}^{\prime} \underset{\sim}{Y}(\rho)
$$

usando (41) e (20) temos que

$$
\left[\left(X_{1}^{\prime} X_{1}\right)^{-}\right]^{\prime}=\left(P^{-}\right)^{\prime}=\frac{1}{a\left(1-\rho^{2}\right)} A \Rightarrow A=a\left(1-\rho^{2}\right)\left[\left(X_{1}^{\prime} X_{1}\right)^{-}\right]^{\prime}
$$

substituindo em (54) vem

$S Q\{$ Periodos e Média $\}=Y_{\sim}^{\prime}(\rho) X_{1}\left[\left(X_{1}^{\prime} X_{1}\right)^{-}\right]^{\prime} X_{1}^{\prime} \underset{\sim}{Y}(\rho)=S Q\left\{X_{1}^{\prime} \underset{\sim}{Y}(\rho)\right\}$

portanto

E SQ $\{$ Períodos e Média $\}=$ posto $\left(X_{1}\right) \sigma_{e}^{2}+\left.\operatorname{SQ}\left\{X_{1}^{\prime} \underset{\sim}{Y}(\rho)\right\}\right|_{\sim \sim \sim}(\rho)=E(\underset{\sim}{Y}(\rho))$

$\operatorname{com} E(\underset{\sim}{Y}(p))=\left.\left(I_{a} \otimes D^{\prime}\right) X\right|_{R(\mu, \underset{\sim}{p})} ^{\underset{\sim}{\beta}} \operatorname{para} \underset{\sim}{\beta^{\prime}}=\left[\mu: p_{1} p_{2} \cdots p_{p}\right]$

e

$$
\begin{aligned}
& \left.\left(I_{a} \otimes D^{\prime}\right) x\right|_{R(\mu, \underset{\sim}{p})}=\frac{1}{\sqrt{1-\rho^{2}}}\left[\underset{\sim}{j}: x_{I}\right] \\
& X_{1}=\left(\sim_{a}^{1} \otimes D^{\prime}\right) . \cdot \operatorname{posto}\left(x_{1}\right)=\text { posto }(D)=p
\end{aligned}
$$

$\left.\operatorname{SQ}\left\{X_{1}^{\prime} \underset{\sim}{Y}(\rho)\right\}\right|_{\underset{\sim}{Y}(\rho)=E(\underset{\sim}{Y}(\rho))}=\left.\left.{\underset{\sim}{\beta}}^{\prime} X^{\prime}\left(I_{a} \otimes D\right)\right|_{R(\mu, \underset{\sim}{p})} X_{1}\left[\left(X_{1}^{\prime} X_{1}\right)^{-}\right]^{\prime} X_{1}^{\prime}\left(I_{a} \otimes D^{\prime}\right) X\right|_{\underset{R}{(\mu, p})} ^{\beta}$

mas por $(40)$

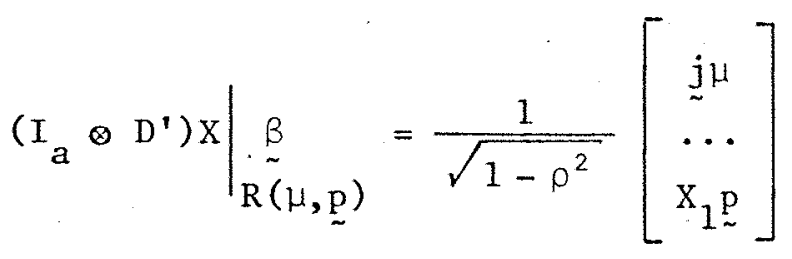

e por (41)

$$
\left.x_{1}^{\prime}\left(I_{a} \otimes D^{\prime}\right) x\right|_{R(\mu, \underset{\sim}{p})} ^{\beta}=\frac{1}{\sqrt{1-\rho^{2}}}\left[\begin{array}{c}
\underset{\sim}{\sim} \\
\ldots \\
\underset{\sim}{P}
\end{array}\right]
$$

Logo 


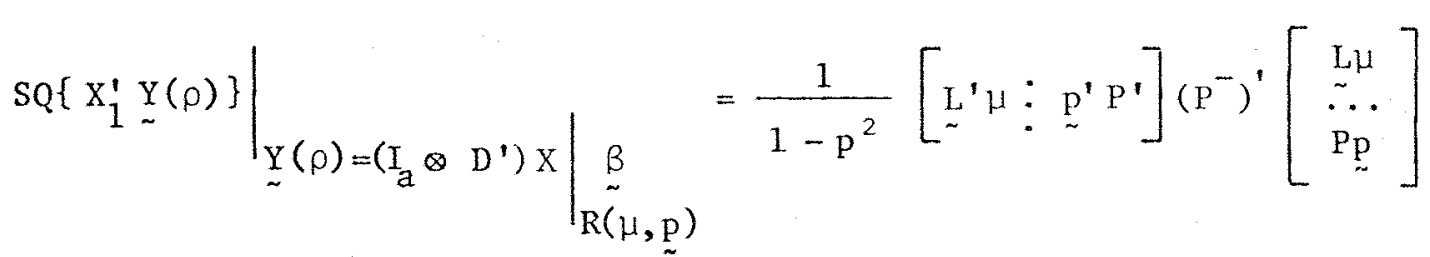

$$
\begin{aligned}
& =\frac{1}{1-\rho^{2}}\left\{\underset{\sim}{L^{\prime}} P^{-1} \underset{\sim}{L} \mu^{2}+\underline{\sim}^{\prime} P \underset{\sim}{p}\right\}
\end{aligned}
$$

pois $\mathrm{P}$ é simétrica e não singular. Logo, usando (20) vem

$$
L_{\sim}^{\prime} P^{-1} L=\frac{1}{a\left(1-\rho^{2}\right)} L_{\sim}^{\prime} A \underset{\sim}{L}
$$

para A e L definidas em (20) e (9) obtemos

$$
L_{\sim}^{\prime} P^{-1} L=a(1-\rho)[p-(p-2) \rho]=c
$$

substituindo em (57) vem

$\left.\operatorname{SQ}\left\{X_{1}^{\prime} \underset{\sim}{Y}(\rho)\right\}\right|_{\underset{\sim}{Y}(\rho)=E(\underset{\sim}{Y}(\rho))}=\frac{1}{1-\rho^{2}}\left\{c \mu^{2}+{\underset{\sim}{p}}^{\prime} P \underset{\sim}{p}\right\}$

levando (56) e (58) em (55) obtemos

E $S Q\{$ Periodos e Mëdia $\}=p \underset{e}{\sigma_{e}^{2}}+\frac{1}{1-\rho^{2}}\left\{c \mu^{2}+\underset{\sim}{p} P \underset{\sim}{p}\right\}$

Por outro 1ado, de (38) temos que a soma de quadrados da média ignorando tratamentos e períodos é:

$$
\mathrm{SQ}\{\text { Média }\}=\frac{1}{c\left(1-\rho^{2}\right)} G^{2}(\rho) \quad \text { com } \quad c=\underset{\sim}{j} \underset{\sim}{j} \text { por }(41)
$$

De (42) temos

$$
G(\rho)=\sqrt{1-\rho^{2}} \quad \underset{\sim}{j^{\prime}} \underset{\sim}{Y}(\rho)
$$

Logo

$$
\operatorname{SQ}\{\operatorname{Média}\}=Y^{\prime}(\rho) \underset{\sim}{\mathbf{j}}\left({\underset{\sim}{j}}^{\prime} \underset{\sim}{j}\right)^{-} \underset{\sim}{\mathbf{j}}{ }_{\sim}^{Y}(\rho)=S Q\left\{\underset{\sim}{\mathbf{j}^{\prime}} \underset{\sim}{Y}(\rho)\right\}
$$




\section{Portanto}

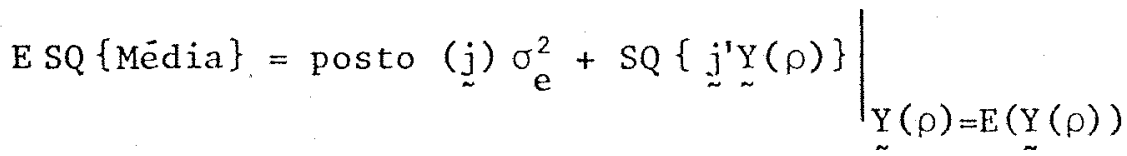

Mas, posto $(\underset{\sim}{j})=1$ e $E(\underset{\sim}{Y}(\rho))=\left.\left(I_{a} \otimes D^{\prime}\right) X\right|_{R} ^{\underset{R}{(\mu)}} \underset{\sim}{\beta}$ para $\underset{\sim}{\beta}=\mu$ e $\left.\left(I_{a} \otimes D^{\prime}\right) X\right|_{R(\mu)}=\frac{1}{\sqrt{1-\rho^{2}}} j$

Logo por (40)

e

$$
\left.\left(I_{a} \otimes D^{\prime}\right) X\right|_{R(\mu)} ^{\underset{B}{\beta}}=\frac{1}{\sqrt{1-\rho^{2}}} \underset{\sim}{j} \mu
$$

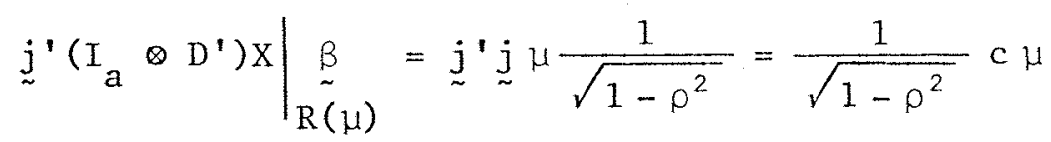

sendo $\left(j^{\prime} \underset{\sim}{j}\right)^{-}=\frac{1}{c}$ obtemos

$$
\operatorname{SQ} \underset{\sim}{j} \underset{\sim}{Y}(\rho)\}=\frac{1}{1-\rho^{2}} c \cdot \mu^{2}
$$

substituindo em (60) obtemos

$$
E S Q\{\text { Média }\}=\sigma_{e}^{2}+\frac{1}{1-\rho^{2}} c \mu^{2}
$$

Portanto, de (59) e (61) podemos obter a esperança matemätica da soma de quadrados de períodos ajustada para a média e ignorando tratamentos, isto é:

$$
\operatorname{ESQ}\{\text { Periodos }\}=(p-1) \sigma_{e}^{2}+\frac{1}{1-\rho^{2}} \underset{\sim}{p^{\prime}} P_{\sim}^{p}
$$

4.1.8. Esperança matemática da soma de quadrados resi dual

De (5) temos

$$
\operatorname{SQ}\{\text { Tota } 1\}={\underset{\sim}{Y}}^{\prime}(\rho) \underset{\sim}{Y}(\rho)=\underset{\sim}{Y^{\prime}}(\rho) I_{\text {ap }} \underset{\sim}{Y}(\rho)
$$




\section{Logo}

$\operatorname{ESQ}\{$ Tota1 $\}=$ posto $\left.\left(I_{a p}\right) \sigma_{e}^{2}+\left.\underline{\sim}^{\prime}(\rho) \underset{\sim}{Y}(\rho)\right|_{\sim}(\rho)=E \underset{\sim}{(Y}(\rho)\right)=\left(I_{a} \otimes D^{\prime}\right) X \underset{\sim}{\beta}$

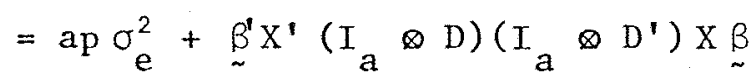

Mas por (41)

$$
\left(I_{a} \otimes D^{\prime}\right) \underset{\sim}{\underset{\beta}{\beta}}=\frac{1}{\sqrt{1-\rho^{2}}}\left[\begin{array}{c}
j \mu \\
\cdots \\
x_{1} p \\
\cdots \\
x_{2} t
\end{array}\right]
$$

Portanto, em (62) obtemos

$$
\operatorname{ESQ}\{\text { Total }\}=a p \sigma_{e}^{2}+\frac{1}{1-\rho^{2}}\left(\underset{\sim}{j} \underset{\sim}{j} \mu^{2}+\underset{\sim}{p^{\prime}} X_{1}^{\prime} X_{1} \underset{\sim}{p}+\underset{\sim}{t} x_{2}^{\prime} X_{2} \underset{\sim}{t}\right)
$$

ou, pela notação de (41)

$$
\operatorname{ESQ}\{\operatorname{Tota} 1\}=a p \underset{\mathrm{e}}{\sigma^{2}}+\frac{1}{1-\rho^{2}}\left(c \mu^{2}+\underset{\sim}{p^{\prime}} P \underset{\sim}{p}+\underline{\sim}^{\prime} \mathrm{T} \underset{\sim}{t}\right)
$$

Por diferença podemos obter a esperança matemática da soma de quadrados do resíduo,

$E S Q\{$ Residuo $\}=E S Q\{$ Total $\}-E S Q\{$ Periodos e Mëdia $\}-E S Q\{$ Tratamentos aj. $\}$

$$
=[a p-p-(K-1)] \sigma_{e}^{2}
$$

ou

$$
\text { E SQ }\{\text { Residuo }\}=[p(a-1)-(K-1)] \sigma_{e}^{2}
$$

uma vez que $\underline{t}^{\prime} \mathrm{C}_{\sim}^{t}=\underline{\sim}^{\prime} \mathrm{T} \underset{\sim}{t}-\underline{\sim}^{\prime} N P^{-1} N^{\prime} \underline{\sim}$ e para $\sum_{k=1}^{K} t_{k}=0$ temos que $N^{\prime} \underset{\sim}{t}=\underline{0}$. 
Os resultados obtidos são sumarizados no quadro que se se gue:

Esperanças matemáticas das somas de quadrados

F. Variação

G.L.

E SQ

Períodos (aj. para Média, ignorando Tratamentos) $\mathrm{p}-1$ $(p-1) \sigma_{e}^{2}+\frac{1}{1-\rho^{2}} \stackrel{\sim}{p}^{\prime} P \underset{\sim}{p}$

Média (ignorando Períodos e Tratamentos)

1 $\sigma_{e}^{2}+\frac{1}{1-\rho^{2}} c \mu^{2}$

Periodos e Média

(ignorando Tratamentos)

$\mathrm{p}$

$p \sigma_{\mathrm{e}}^{2}+\frac{1}{1-p^{2}}\left(\mathrm{c} \mu^{2}+\underset{\sim}{\mathrm{p}} \mathrm{p} \underset{\sim}{\mathrm{p}}\right)$

Tratamentos (ajustado)

$K-1 \quad(K-1) \sigma_{e}^{2}+\frac{1}{1-\rho^{2}}\left[\frac{a(p-2)}{K} \rho^{2}+\Lambda \rho+r\right] \sum_{k=1}^{K} t_{k}^{2}$

Residuo

$$
p(a-1)-(k-1) \quad[p(a-1)-(k-1)] \sigma_{e}^{2}
$$

Total

ap $\quad$ ap $\sigma_{e}^{2}+\frac{1}{1-\rho^{2}}$

$\left(\mathrm{c} \mu^{2}+\underset{\sim}{p} \mathrm{P} \underset{\sim}{\mathrm{p}}+\underset{\sim}{t^{\prime}} \mathrm{T} \underset{\sim}{t}\right)$

4.2. Variāncia de estimativas de efeitos de tratamentos

De (43) temos que

$$
\underset{\sim}{Q}(\rho)=H^{\prime} \underset{\sim}{Y}(\rho) \sqrt{1-\rho^{2}}
$$

onde $H^{\prime}=\mathrm{X}_{2}^{\prime}-\mathrm{NP}^{-1} \mathrm{X}_{1}^{\prime}$

Logo

$$
\operatorname{Var}\{\underset{\sim}{Q}(\rho)\}=\left(1-\rho^{2}\right) H^{\prime} \operatorname{Var}\{\underset{\sim}{Y}(\rho)\} H
$$

por (6) $\operatorname{Var}\{\underline{\sim}(\rho)\}=I_{\text {ap }} \sigma_{e}^{2}$ 
portanto

$$
\operatorname{Var}\{\underset{\sim}{Q}(\rho)\}=\left(1-\rho^{2}\right) \mathrm{H}^{\prime} \mathrm{I}_{\mathrm{ap}} \mathrm{H} \sigma_{\mathrm{e}}^{2}
$$

ou

$$
\operatorname{Var}\{\underset{\sim}{Q}(\rho)\}=\left(1-\rho^{2}\right) H^{\prime} H \sigma_{e}^{2}
$$

usando (47) temos

$$
\operatorname{Var}\{\underset{\sim}{Q}(\rho)\}=\left(1-\rho^{2}\right) C \sigma_{e}^{2}
$$

Por outro lado, de (45)

$$
\underset{\sim}{\hat{t}}=C^{-} \underset{\sim}{Q}(\rho)
$$

Logo

$$
\operatorname{Var}\{\underline{\underline{E}}\}=C^{-} \operatorname{Var}\{\underset{\sim}{Q}(p)\}\left(C^{-}\right)^{\prime}
$$

usando (63) vem

$$
\operatorname{Var}\{\underset{\sim}{\hat{E}}\}=\left(1-\rho^{2}\right) C^{-} C\left(C^{-}\right)^{\prime} \sigma_{e}^{2}
$$

Portanto, se $\underset{\sim}{Z^{\prime}} t$ é estimáve1, isto $\bar{e}, \underset{\sim}{Z^{\prime}}=\underset{\sim}{\alpha^{\prime}} \mathrm{C}$, então

$$
\operatorname{Var}\left\{\underset{\sim}{Z^{\prime}} \hat{E}\right\}=\underset{\sim}{Z^{\prime}} \operatorname{Var}\{\underline{\tilde{E}}\} \underset{\sim}{Z}
$$

Usando (64) temos

$$
\operatorname{Var}\left\{\underset{\sim}{Z^{\prime}} \underline{\tilde{E}}\right\}=\underset{\sim}{Z^{\prime}} C^{-} C\left(C^{-}\right)^{\prime} \underset{\sim}{Z}\left(1-\rho^{2}\right) \sigma_{e}^{2}
$$

Para $\underset{\sim}{Z}=C_{\sim}^{\alpha}$, pois $C=C^{\prime}$, vem

$$
\begin{aligned}
\operatorname{Var}\left\{\underset{\sim}{Z^{\prime} E}\right\} & =\underset{\sim}{Z^{\prime}} C^{-} C\left(C^{-}\right)^{\prime} C \underset{\sim}{\alpha}\left(1-\rho^{2}\right) \sigma_{e}^{2} \\
& =\underset{\sim}{Z^{\prime}} C^{-} C \underset{\sim}{\alpha}\left(1-\rho^{2}\right) \sigma_{e}^{2} \\
& =Z_{\sim}^{\prime} C^{-} \underset{\sim}{Z}\left(1-p^{2}\right) \sigma_{e}^{2}
\end{aligned}
$$


e para $\underset{\sim}{Z^{\prime}}=\left[\begin{array}{lllllll}0 & \ldots & 1 & \ldots & -1 & \ldots & 0\end{array}\right]$ temos

$$
\operatorname{Var}\left\{\bar{t}_{k}-\bar{t}_{k^{\prime}}\right\}=\frac{2 \sigma_{e}^{2}\left(1-\rho^{2}\right)}{\frac{a(p-2)}{k} \rho^{2}+\Lambda \rho+r}
$$

4:3. Exemplo ilustrativo da análise da variāncia pelo mode lo auto-regressivo

Como ilustração, consideremos o caso de $\mathrm{K}=3$ tratamentos e $p=3$ períodos, adotando-se as observações da Tabela 1, relativas aos 6 primeiros animais e os 3 primeiros períodos, obtendo o seguinte quadro de dados.

Produção média diäria de leite em $\mathrm{kg}$ e estatísticas básicas para anälise da variancia pelo modelo auto-regressivo com $\rho=0,9$ e $t_{1}=t_{2}=t_{3}=0$

\begin{tabular}{|c|c|c|c|c|}
\hline \multirow{2}{*}{ Anima is } & \multicolumn{3}{|c|}{ Períodos } & \multirow{2}{*}{ Totais } \\
\hline & $\mathrm{P}_{1}$ & $\mathrm{P}_{2}$ & $\mathrm{P}_{3}$ & \\
\hline 1 & 9,64 (1) & $9,88 \quad(2)$ & $7,96 \quad(3)$ & - \\
\hline 2 & $13,95 \quad(2)$ & 13,13 & $10,49(1)$ & - \\
\hline 3 & $8,26 \quad(3)$ & 7,69 (1) & $7,50 \quad(2)$ & - \\
\hline 4 & $8,75^{\prime}(1)$ & $8,48 \quad(3)$ & $8,37(2)$ & - \\
\hline 5 & $9,38 \quad(2)$ & $8,55 \quad(1)$ & $7,43(3)$ & - \\
\hline 6 & 17,07 (3) & $14,61(2)$ & $14,30(1)$ & - \\
\hline$P_{j 1}$ & 17,64 & 46,10 & 14,93 & 82,67 \\
\hline$P_{j 2}$ & 26,71 & 37,85 & 22,26 & 86,82 \\
\hline$P_{j 3}$ & 22,70 & 40,73 & 18,86 & 82,29 \\
\hline$P_{j}$ & 67,05 & 62,34 & 56,05 & - \\
\hline $\mathrm{P}_{j}(\rho)$ & 10,9440 & 2,0454 & $-0,0560$ & - \\
\hline $\mathrm{T}_{1}^{(\mathrm{j})}$ & 18,39 & 16,24 & 24,79 & $\mathrm{~T}_{1}=59,42$ \\
\hline $\mathrm{T}_{2}^{(j)}$ & 23,33 & 24,49 & 15,87 & $\mathrm{~T}_{2}=63,69$ \\
\hline$T_{3}^{(j)}$ & 25,33 & 21,61 & 15,39 & $\mathrm{~T}_{3}=62,33$ \\
\hline
\end{tabular}


No esquema temos

$$
a=6 ; \quad r=6 ; \quad k=p=3 \text { e } \Lambda=\frac{2 r(p-1)}{p(K-1)}=4
$$

$\operatorname{com} P_{j k} ; P_{j}(\rho)$ e $T_{k}^{(j)}$ definidos em 4.1.2

e

$$
\begin{aligned}
& G(\rho)=(1-\rho) P_{1}+(1-\rho)^{2} \sum_{j=2}^{p-1} P_{j}+(1-\rho) P_{p}=12,9334 \\
& T_{k}(\rho)=T_{k}-\rho \sum_{j=1}^{p} P_{j k}+\rho^{2} \sum_{j=2}^{p-1} T_{k}(j)
\end{aligned}
$$

ou seja

$$
\begin{aligned}
& \mathrm{T}_{1}(\rho)=59,42-0,9 \cdot(78,67)+0,81 \cdot(16,24)=1,7714 \\
& \mathrm{~T}_{2}(\rho)=63,69-0,9 \cdot(86,82)+0,81 \cdot(24,49)=5,3889 \\
& \mathrm{~T}_{3}(\rho)=62,33-0,9 \cdot(82,29)+0,81 \cdot(21,61)=5,7731 \\
& \mathrm{Q}_{k}(\rho)=\mathrm{T}_{k}(\rho)-\frac{1}{\mathrm{~K}} \mathrm{G}(\rho)
\end{aligned}
$$

ou seja

$$
\begin{aligned}
& Q_{1}(\rho)=1,7714-\frac{1}{3} 12,9334=-2,5397 \\
& Q_{2}(\rho)=5,3889-\frac{1}{3} 12,9334=1,0778 \\
& Q_{3}(\rho)=5,7731-\frac{1}{3} 12,9334=1,4620
\end{aligned}
$$

Portanto,

SQ $\{$ Média (ignor. Trat. e Per.) $\}=\frac{G^{2}(\rho)}{a(1-\rho)\left(1-\rho^{2}\right)[p-(p-2) \rho]}=698,7169$

SQ $\{$ Periodos e Média (ignor. Trat.) $\}=\frac{1}{a\left(1-\rho^{2}\right)} \sum_{j=1}^{p} P_{j} P_{j}(\rho)=752,7778$ donde

SQ $\{$ Períodos (aj. para Média, ignor. Trat.) $\}=54,0609$ 
$S Q\{$ Tratamentos aj. $\}=\frac{1}{\left[\frac{a(p-2)}{K} \rho^{2}+\Lambda \rho+r\right]\left(1-\rho^{2}\right)} \sum_{k=1}^{K} Q_{k}^{2}(\rho)=4,5732$

$S Q\{$ Total $\}=\frac{1}{1-\rho^{2}} \sum_{i=1}^{a}\left\{\left(1-\rho^{2}\right) y_{i 1}^{2}+\sum_{j=2}^{p}\left(y_{i j}-\rho y_{i(j-1)}\right)^{2}\right\}$

$$
=\frac{1}{0,19}\{0,19 \cdot(811,6915)+2,7905+4,9045\}=852,1915
$$

por diferença obtemos a SQ Residuo\} e o quadro da anälise da variância será:

\begin{tabular}{lrrrrr}
\hline F. Variação & G.L. & SQ & QM & F & N.M.S. \\
\hline Média & 1 & 698,7169 & & & \\
Períodos & 2 & 54,0609 & & & \\
Tratamentos aj. & 2 & 4,5732 & 2,2866 & 0,3134 & $\simeq 73 \%$ \\
Residuo & 13 & 94,8405 & 7,2954 & & \\
\hline Total & 18 & 852,1915 & & \\
\hline \hline
\end{tabular}

Portanto, o valor de $F$ nos leva à não rejeição da hipótese de que $t_{1}=t_{2}=t_{3}=0$, como era de se esperar. Em GODOI (1983) o autor utiliza os mesmos dados deste exemplo ilustrativo, e obtém para o modelo auto-regressivo no esquema de reversão um valor $F=1,0369 \mathrm{com} 2$ e $13 \mathrm{~g} .1$. correspondendo a um N.M.S. $\simeq 37 \%$, enquanto que para o modelo "switchback" obtém $F=0,8180$ com 2 e $3 \cdot$ g.1. correspondendo a um N.M.S. $\simeq 52 \%$.

Se considerarmos $t_{1}=-0,5 \mathrm{~kg} ; t_{2}=0,0 \mathrm{~kg}$ e $t_{3}=0,5 \mathrm{~kg} \mathrm{e}$ adcionarmos estes valores às observações respectivas do quadro anterior, obtemos 


\begin{tabular}{crrr}
\hline & \multicolumn{4}{c}{ Periodos } \\
\cline { 2 - 4 } Animais & $\mathrm{P}_{1}$ & \multicolumn{1}{c}{$\mathrm{P}_{2}$} & \multicolumn{1}{c}{$\mathrm{P}_{3}$} \\
\hline 1 & $9,14(1)$ & $9,88(2)$ & $8,46(3)$ \\
2 & $13,95(2)$ & $13,63(3)$ & $9,99(1)$ \\
3 & $8,76(3)$ & $7,19(1)$ & $7,50(2)$ \\
4 & $8,25(1)$ & $8,98(3)$ & $8,37(2)$ \\
5 & $9,38(2)$ & $8,05(1)$ & $7,93(3)$ \\
6 & $17,57(3)$ & $14,61(2)$ & $13,80(1)$ \\
\hline \hline
\end{tabular}

e utilizando as mesmas fórmulas anteriores, obtemos o quadro da anälise da variância que se segue:

\begin{tabular}{lrrrrr}
\hline F. Variaça & G.L. & SQ & QM & F & N.M.S. \\
\hline Mëdia & 1 & 698,7169 & & & \\
Periodos & 2 & 54,0609 & & & \\
Tratamentos aj. & 2 & 70,6612 & 35,3306 & 4,8429 & $\approx 2,7 \%$ \\
Residuo & 13 & 94,8405 & 7,2954 & & \\
\hline Total & 18 & 918,2795 & & & \\
\hline \hline
\end{tabular}

Portando, o valor de $\mathrm{F}$ nos leva à rejeição da hipótese que $t_{1}=t_{2}=t_{3}=0$, como era de se esperar. Já em GODOI (1983), os resulta dos obtidos pelo autor foram: para o modelo auto-regressivo no esquema de reversão $F=6,0473$ (N.M.S. $=1,3 \%$ ) e para o modelo "switchback" $F=5,39$ (N.M.S. = 10\%). Assim, a adoção do esquema "change-over" para o modelo auto-regressivo se apresenta mais sensivel que o modelo usual de Lucas, como será verificado na seqüència deste trabalho. 
4.4. Comparação da sensibilidade dos modelos e adequação do modelo auto-regressivo

Nas Tabelas de 4 a 15 são apresentados os resultados das anālises, baseados nos quais podemos discutir a adequação do modelo autoregressivo às vārias situações experimentais, bem como comparar a sensibi lidade na detecção de efeitos de tratamentos pelos modelos:

(a) "Switchback"

(b) Rotativo no esquema em quadrados latinos

(c) Auto-regressivo com $\mathrm{p}=3$ periodos

(d) Auto-regressivo com $\mathrm{p}=\mathrm{K}$

Nas anälises efetuadas através do modelo do delineamento rotativo (b), para cada valor de $\mathrm{K}$ considerado, não mais que $5 \%$ delas apre sentaram interação (Tratamento $\times$ Quadrados Latinos) significativa, o que nos leva à não rejeição da hipótese de ausencia desta interação e conse qüente adoção do residuo médio como estimador do erro amostral para este modelo.

Na Tabela 4 são apresentadas as mëdias das distribuições dos niveis minimos de significância (N.M.S.) para os värios ensaios na au sēncia de efeitos de tratamentos, bem como os respectivos valores da esta tistica $D$ do teste de aderência de Kolmogorov-Smirnov, da distribuição dos N.M.S. à distribuição uniforme no intervalo $[0,1]$. Nesta tabela ob serva-se uma boa adequação dos värios modelos às condições experimentais e pode-se verificar para o modelo auto-regressivo uma tendéncia em reje tar indevidamente a hipótese de ausência de efeitos de tratamentos, à me dida que o valor de $\rho$ adotado supera o populacional 0,91. Por outro 1ado, para valores de $\rho$ inferiores ao populacional, constata-se uma assimetria à direita na distribuição dos N.M.S. 
Nas Tabelas 5, 6 e 7 são apresentadas as médias e desvios padrões das distribuições dos N.M.S. na presença de efeitos aditivos de tratamentos. Nestas tabelas pode-se verificar a superioridade dos mode los auto-regressivos (c) e (d) em relação aos usuais (a) e (b), no que se refereà sensibilidadena detecção de contrastes de tais efeitos. Para os modelos (c) e (d) percebe-se que tal sensibilidade aumenta em função do valor de p adotado, e que se tal valor supera o populacional existe uma tendência de se detectar mais diferenças significativas do que realmente existem, em função dos resultados apresentados na Tabela 4 .

Comparando-se os resultados obtidos para os modelos autoregressivos (c) e (d), percebe-se que a sensibilidade aumenta em função do numero de períodos adotados, indicando assim que o caso en que $\mathrm{p}=\mathrm{K}$ deve ser preferivel ao caso em que $\mathrm{p}<\mathrm{K}$.

Nas Tabelas 8,9 e 10 são apresentadas as distribuições de frequēncias para as quatro classes iniciais, dos 100 N.M.S. para cada en saio, considerando-se efeitos não todos nulos de tratamentos. Também des tas tabelas podemos comprovar a superioridade dos modelos (c) e (d) na de tecção de contrastes entre tais efeitos, uma vez que as frequéncias dos N.M.S. nas classes iniciais são maiores para estes modelos do que para os usuais (a) e (b). Percebe-se porém que o modelo rotativo (b) é mais sen sível que o auto-regressivo com $p=3$ períodos (c), quando $K=4$, sendo que tal superioridade inexiste para $K=5$. Esse resultado vem mais uma vez confirmar que o modelo auto-regressivo deve ser preferível com número máximo de períodos, isto é $\mathrm{p}=\mathrm{K}$. Entretanto, em se comparando com o mo delo "switchback" (a), a sensibilidade é maior para o auto-regressivo mes mo $\operatorname{com} \mathrm{p}=3$ periodos. 
Na Tabela 11 são apresentadas as médias e variāncias dos 100 valores da estatistica $F$, obtidas nas anälises da variancia para os vários ensaios com auséncia de efeitos de tratamentos. Observa-se nesta tabela que os resultados obtidos são bastante próximos dos valores espera dos das distribuições $F$ correspondentes, os quais também são apresentados nesta tabela, indicando, portanto, uma boa adequação dos modelos. Obser va-se ainda que, para os modelos auto-regressivos, o valor de $\mathrm{F}$ é propor cional ao valor do coeficiente $\rho$ adotado, sendo que para $\rho$ em torno de 0,91 é que se obtēm uma melhor adequação. Estes resultados sugerem que, variando o valor de $\rho$, os valores de $\mathrm{F}$ passam de subestimativas para supe restimativas do valor exato.

Nas Tabelas 12, 13 e 14 são apresentados os quocientes de médias de 100 anälises do QM Trat: (aj.) pelo QM Res., para os värios en saios com efeitos não todos nulos de tratamentos. Para os modelos autoregressivos observa-se que os valores obtidos são muito próximos dos espe rados segundo a expressão

$$
\frac{E(Q M T)}{E(Q M R)}=1+\left[\frac{a(p-2)}{k} \rho^{2}+\Lambda \rho+r\right] \frac{\sum_{k=1}^{K} t_{k}^{2}}{(K-1)\left(1-\rho^{2}\right) \sigma_{e}^{2}}
$$

onde $\sigma_{e}^{2}=6,00$ é adotado e indicado por GODOI (1983) para os dados da Tabela 1, segundo o modelo auto-regressivo. Observa-se ainda nestas tabe las que os valores dos quocientes passam de superestimativas a subestima tivas dos valores esperados, a medida que se aumenta o valor do coeficien te $\rho$, sendo que, em geral, para $\rho$ em torno de 0,91 se obtém resultados mais concordantes.

$\mathrm{Na}$ Tabela 15 são apresentadas as mëdias e os desvios pa dröes dos 100 valores do Quadrado Médio Residual para os värios ensaios, 
donde se pode inferir que os modelos auto-regressivos (c) e (d) com $\rho$ em torno de 0,91 apresentam resultados concordantes com o valor populacional $\sigma_{\mathrm{e}}^{2}=6,00$. Percebe-se ainda que a variancia residual passa de subestima da para superestimada, conforme se aumenta o valor de $\rho$, o que leva a re sultados concordantes com os apresentados nas tabelas anteriores.

Por outro lado, podemos notar que alguns resultados são concordantes com os que se podem inferir da expressão (65) relativa à variancia de um contraste entre dois tratamentos. De fato, nesta expres são observamos que aumentando o valor de p e/ou o valor de $\rho$, diminui a variancia, o que implica em uma maior sensibilidade para a deteção des tes contrastes. Ademais se $\rho<0$, o que em geral não ocorre em experimen tos com vacas em lactação, a variāncia é maior do que se $\rho \geq 0$, resultan do uma menor sensibilidade do modelo. 


\section{CONCLUSÕES}

Em função dos resultados obtidos e apresentados no capítu Io anterior, destacam-se as seguintes conclusões:

(a) O modelo auto-regressivo com $3 \leq \mathrm{p} \leq \mathrm{K}$ é adequado para experimentos com vacas em lactação, desde que o coeficiente de correlação linear entre periodos possa ser obtido de lactações anteriores de animais que poderão ser amostrados para o ensaio;

(b) Se tal coeficiente éxceder o populacional, haverà uma tendencia indevida, do modelo, de detectar mais diferenças de efeitos de tratamentos, enquanto que a sensibilidade diminui se utilizarmos um valor menor que o populacional;

(c) Em condições ideais, isto é, com o verdadeiro valor de $\rho$, os modelos auto-regressivos com $p=3$ e $p=K$ são mais sensiveis do que os modelos dos delineamentos "switchback" e rotativo, respectivamente;

(d) Deve-se dar preferência ao modelo auto-regressivo com $\mathrm{p}=\mathrm{K}$, pois a sensibilidade do modelo se mostrou proporcional ao número de periodos; 
(e) Aumentando-se os valores de $\rho$, a variancia residual pas sa de subestimada a superestimada e conseqüentemente os valores da esta tistica $F$ passam de superestimativas a subestimativas da mëdia da distri buição $\mathrm{F}$ correspondente;

(f) O modelo auto-regressivo se mostra sensivel a pequenas variações no valor adotado do coeficiente de correlação linear entre pe ríodos, o que exigirà que este seja conhecido ou bem estimado;

(g) Os resultados sugerem que se proceda a investigação do efeito da estimação do coeficiente $\rho$ na sensibilidade do modelo auto-re gressivo, bem como a continuidade nesta linha de pesquisa considerando a estimação do efeito residual de um tratamento sobre outro aplicado no pe ríodo subseqüente. 


\section{LITERATURA CITADA}

BRANDT, A.E., 1938. Tests of significance in reversa1 or switchback trial. Research Bulzetin 234. Agr. Exp. Sta. Ames, Iowa.

COCHRAN, W.G. e G.M. COX, 1957. Experimental designs. 2a Ed., Nova York, John Wiley \& Sons. $611 \mathrm{p}$.

COCHRAN, W.G.; K.M. AUTREY e C.Y. CANNON, 1941. A double change-over design for dairy cattle feeding experiments. J. Daimy Sci., 24:937-951.

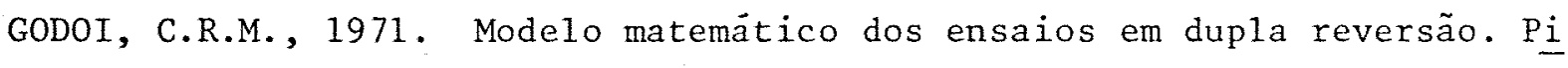
racicaba, ESALQ/USP, 52 p. (Tese de Doutoramento).

GODOI, C.R.M., 1972. Anālise da variāncia dos ensaios em "switchback" pa ra animais quando há perda de parcela: correção da tendenciosidade da soma de quadrados de tratamentos. Anais da VIII Reunião da SBZ. p. 143. GODOI, C.R.M., 1983. Modelo linear com auto-correlação nos resíduos para anālise de produção leiteira em experimentos com dupla reversão. Pira cicaba, ESALQ/USP, $110 \mathrm{p}$. (Tese de Livre Docência).

GODOI, C.R.M. e I.R. NOGUEIRA, 1972. Mode1o matemático dos ensaios em du pla reversão para experimentos com animais. Anais da VIII Reunião da SBZ. p. 145 . 
HOFFMANN, R. e S. VIEIRA, 1977. AnäZise de regressão. Hucitec-Edusp, São Paulo. 339 p.

KENNEDY, W. e J.E. GENTLE, 1980. Statistical computing. Nova York, Mar cel Dekker. 589 p.

LUCAS, H.L., 1956. Switchback trials for more than two treatments. J. Dairy Sci., 39:1-7.

LUCAS, H.L., 1960. Critica1 features of good dairy feeding experiments. J. Dairy Sci., 43:193-212.

MOOD, A.M.; F.A. GRAYBILL, D.C. BOES, 1974. Introduction to the theory of statistics. 3ạ ed., Nova York, McGraw-Hill. 564 p.

PATTERSON, H.D., 1950. The analysis of change-over trials. J. Agric. Sci., 40: $375-380$.

PATTERSON, H.D., 1952. The construction of balanced designs for experi ments involving sequences of treatments. Biometrika, 39:32-48. PATTERSON, H.D. e H.L. LUCAS, 1962. Change-over designs. Tech. BuZ. 14?, North Carolina Agric. Exper. Station and U.S. Department of Agric. RAO, C.R., 1965. Linear statistical inference and its aplications. Nova York, John Willey \& Sons. 522 p.

SCHEFFE, H., 1959. The analysis of variance. Nova York, John Willey \& Sons. 477 p.

SNEDECOR, G.W., 1946. Statistical methods. 4a ed. Iowa State College Press, Ames. 593 p.

TAYLOR, W.B. e P.J. ARMSTRONG, 1953. The efficiency of some experimenta1 designs used in dairy husbandry experiments. J. Agric. Sci., 43:407412.

THEIL, H., 1971. Principles of econometrics. Nova York, John Willey \& Sons. $735 \mathrm{p}$. 
WILLIANS, E.J., 1949. Experimental designs balanced for the estimation of residual effects of treatments. Austr. T. Sci. Res., 2(A):149. 
7. TABELAS 
TABELA 1 - Médias (em $\mathrm{kg}$ ) de períodos de 15 dias da produção leiteira de vacas da raça holandesa.

\begin{tabular}{|c|c|c|c|c|c|c|c|}
\hline \multirow{2}{*}{ VACAS } & \multicolumn{7}{|c|}{ PERIOODOS } \\
\hline & 1. & 2 & 3 & 4 & 5 & 6 & 7 \\
\hline 1 & 9,64 & 9,88 & 7,96 & 6,40 & 6,84 & 6,12 & 5,79 \\
\hline 2 & 13,95 & 13,13 & 10,49 & 10,67 & 9,64 & 9,00 & 8,25 \\
\hline 3 & 8,26 & 7,69 & 7,50 & 8,08 & 7,97 & 6,45 & 6,20 \\
\hline 4 & 8,75 & 8,48 & 8,37 & 8,18 & 7,36 & 6,82 & 7,65 \\
\hline 5 & 9,38 & 8,55 & 7,43 & 6,98 & 6,25 & 6,02 & 5,80 \\
\hline 6 & 17,07 & 14,61 & 14,30 & 13,36 & 13,94 & 12,44 & 10,69 \\
\hline 7 & 11,94 & 10,74 & 10,22 & 9,33 & 9,37 & 8,04 & 7,97 \\
\hline 8 & 10,79 & 10,56 & 10,00 & 8,13 & 10,84 & 8,76 & 9,04 \\
\hline 9 & 8,56 & 7,32 & 7,74 & 6,71 & 6,88 & 6,77 & 5,19 \\
\hline 10 & 9,01 & 9,14 & 8,58 & 8,54 & 6,49 & 5,99 & 5,36 \\
\hline 11 & 10,02 & 10,91 & 10,22 & 8,80 & 6,58 & 6,34 & 5,84 \\
\hline 12 & 12,41 & 12,38 & 12,78 & 13,05 & 11,35 & 11,86 & 11,73 \\
\hline 13 & 7,18 & 7,25 & 6,59 & . 7,22 & 5,45 & 4,79 & 4,74 \\
\hline 14 & 7,20 & 6,79 & 5,48 & 4,68 & 3,75 & 3,64 & 4,17 \\
\hline 15 & 8,58 & 7,94 & 7,11 & 4,97 & 3,64 & 3,75 & 4,24 \\
\hline 16 & 7,13 & 6,62 & 5,77 & 5,30 & 5,16 & 5,12 & 5,32 \\
\hline 17 & 8,92 & 8,53 & 8,45 & 10,99 & 8,33 & 6,86 & 8,11 \\
\hline 18 & 11,35 & 10,79 & 9,30 & 9,42 & 9,41 & 8,41 & 8,86 \\
\hline 19 & 7,83 & 6,45 & 6,13 & 6,06 & 5,64 & 5,19 & 5,09 \\
\hline 20 & 8,82 & 8,43 & 7,77 & 7,11 & 5,33 & 5,33 & 5,15 \\
\hline 21 & 11,46 & 9,54 & 7,82 & 7,28 & 7,46 & 5,74 & 4,85 \\
\hline 22 & 6,45 & 6,20 & 5,54 & 5,10 & 4,72 & 5,55 & 5,42 \\
\hline 23 & 11,79 & 12,53 & 10,71 & 9,30 & 9,60 & 7,51 & 5,89 \\
\hline 24 & 7,25 . & 7,50 & 7,29 & 7,25 & 7,50 & 6,67 & 7,13 \\
\hline
\end{tabular}


TABELA 1 - Continuação.

\begin{tabular}{|c|c|c|c|c|c|c|c|}
\hline \multirow{2}{*}{ VACAS } & \multicolumn{7}{|c|}{ PERIODOS } \\
\hline & 1 & 2 & 3 & 4 & 5 & 6 & 7 \\
\hline 25 & 7,26 & 7,34 & 7,20 & 7,46 & 8,49 & 7,73 & 6,86 \\
\hline 26 & 10,27 & 8,95 & 7,90 & 7,13 & 7,41 & 7,17 & 6,60 \\
\hline 27 & 10,38 & 9,50 & 7,90 & 7,25 & 7,66 & 6,88 & 7,55 \\
\hline 28 & 10,41 & 9,49 & 9,14 & 8,62 & 9,96 & 9,26 & 8,33 \\
\hline 29 & 8,21 & 7,48 & 8,36 & 7,90 & 8,16 & 7,17 & 6,29 \\
\hline 30 & 12,22 & 11,34 & 10,71 & 9,79 & 9,22 & 9,76 & 9,23 \\
\hline 31 & 12,62 & 11,24 & 9,94 & 10,69 & 9,53 & 8,75 & 7,63 \\
\hline 32 & 12,20 & 10,89 & 10,75 & 9,99 & 9,56 & 8,24 & 8,46 \\
\hline 33 & 9,78 & 9,75 & 8,42 & 8,25 & 7,84 & 8,20 & 8,23 \\
\hline 34 & 9,86 & 9,68 & 8,66 & 7,34 & 6,70 & 6,48 & 5,62 \\
\hline 35 & 11,88 & 9,28 & 10,17 & 9,39 & 8,72 & 9,68 & 6,25 \\
\hline 36 & 13,87 & 10,86 & 9,78 & 9,59 & 9,30 & 9,76 & 9,28 \\
\hline 37 & 10,10 & 10,08 & 9,06 & 8,04 & 7,66 & 7,54 & 5,47 \\
\hline 38 & 7,68 & 7,49 & 7,79 & 8,42 & 6,26 & 5,97 & 5,99 \\
\hline 39 & 6,70 & 7,12 & 5,59 & 5,56 & 6,79 & 5,59 & 4,82 \\
\hline 40 & 9,19 & 7,61 & 6,38 & 6,89 & 6,69 & 6,26 & 5,90 \\
\hline 41 & 14,39 & 14,68 & 13,57 & 14,06 & 12,43 & 13,22 & 12,33 \\
\hline 42 & 12,93 & 11,20 & 10,68 & 9,80 & 9,39 & 8,52 & 7,60 \\
\hline 43 & 14,03 & 14,78 & 12,15 & 10,71 & 8,94 & 8,34 & 7,72 \\
\hline 44 & 17,79 & 14,89 & 14,51 & 13,45 & 13,13 & 13,06 & 12,53 \\
\hline 45 & 5,97 & 7,01 & 4,44 & 5,73 & 4,98 & 3,88 & 3,59 \\
\hline 46 & 7,91 & 5,38 & 4,54 & 5,05 & 4,54 & 4,72 & 5,21 \\
\hline 47 & 8,76 & 8,81 & 8,11 & 6,99 & 7,59 & 6,88 & 6,32 \\
\hline 48 & 8,74 & 9,26 & 8,89 & 7,40 & 6,53 & 5,49 & 5,86 \\
\hline
\end{tabular}


TABELA 1 - Continuação.

PERIODOS

\begin{tabular}{|c|c|c|c|c|c|c|c|}
\hline \multirow{2}{*}{ VACAS } & \\
\hline & 1 & 2 & 3 & 4 & 5 & 6 & 7 \\
\hline 49 & 8,20 & 8,12 & 8,03 & 7,35 & 6,07 & 5,95 & 6,47 \\
\hline 50 & 10,83 & 10,04 & 10,34 & 12,63 & 12,92 & 10,29 & 11,13 \\
\hline 51 & 11,70 & 10,81 & 10,11 & 10,37 & 9,90 & 9,59 & 8,95 \\
\hline 52 & 10,66 & 11,51 & 10,00 & 8,50 & 7,67 & 7,96 & 7,44 \\
\hline 53 & 11,60 & 13,40 & 11,50 & 10,13 & 9,41 & 9,57 & 8,64 \\
\hline 54 & 9,76 & 11,30 & 9,08 & 7,56 & 6,45 & 7,40 & 5,48 \\
\hline 55 & 7,51 & 8,46 & 8,37 & 8,15 & 8,20 & 9,31 & 9,46 \\
\hline 56 & 7,55 & 7,35 & 6,72 & 7,99 & 6,32 & 6,54 & 5,86 \\
\hline 57 & 9,97 & 8,37 & 7,86 & 5,99 & 6,04 & 7,67 & 8,18 \\
\hline 58 & 9,37 & 9,97 & 9,64 & 9,01 & 9,08 & 9,00 & 8,67 \\
\hline 59 & 14,36 & 12,62 & 11,80 & 11,33 & 11,75 & 10,57 & 9,80 \\
\hline 60 & 13,70 & 13,64 & 12,18 & 11,39 & 12,09 & 11,12 & 10,47 \\
\hline 61 & 10,11 & 9,02 & 9,97 & 8,90 & 8,02 & 7,77 & 10,45 \\
\hline 62 & 12,77 & 12,87 & 12,47 & 11,72 & 13,45 & 12,96 & 11,74 \\
\hline 63 & 8,38 & 9,15 & 7,88 & 8,67 & 8,04 & 7,39 & 8,51 \\
\hline 64 & 12,97 & 10,53 & 11,81 & 11,49 & 9,02 & 9,70 & 11,84 \\
\hline 65 & 11,33 & 9,76 & 9,48 & 9,29 & 8,11 & 7,41 & 9,34 \\
\hline 66 & 17,09 & 15,13 & 13,85 & 12,47 & 10,86 & 11,40 & 12,45 \\
\hline 67 & 12,26 & 11,96 & 9,87 & 9,77 & 8,68 & 7,08 & 9,59 \\
\hline 68 & 13,43 & 12,54 & 12,21 & 11,77 & 9,83 & 9,67 & 11,06 \\
\hline 69 & 9,44 & 8,57 & 8,50 & 8,01 & 7,06 & 7,23 & 7,71 \\
\hline 70 & 9,01 & 8,62 & 8,68 & 7,82 & 7,68 & 7,89 & 8,13 \\
\hline 71 & 7,90 & 7,43 & 7,23 & 8,09 & 8,42 & 9,16 & 10,05 \\
\hline 72 & 10,95 & 10,91 & 7,63 & 7,52 & 8,14 & 8,29 & 9,38 \\
\hline
\end{tabular}


TABELA 1 - Continuação.

VACAS

PERIODOS

12

8,85

9,07

74

8,08

8,36

95

$11,45 \quad 13,58$

96
15,76

13,97
13,02
8,74

7,64

7,52

8,57

8,51

8,52

7,64

8,22

8,70

9,35

7,64

9,34

15,03

15,70

12,02

12,44

$11,30 \quad 10,58$

8,48

8,65

9,61

12,17

9,30

9,77

$9,14 \quad 10,31$

8,66

10,40

11,39

9,38

10,99

11,76

9,45

10,80

11,76

11,66

9,67

10,95

$11,47 \quad 10,16$

9,62

9,08

10,15

7,39

8,18

7,53

11,12

9,67

8,10

8,58

12,69

12,76

15,62

12,99

14,31

14,62

7

7,50

8,28

8,34

14,42

13,04

9,46

7,37

11,01

10,40

10,76

12,97

9,54

12,21

10,06

10,11

9,84

$9,96 \quad 10,50$

$9,50 \quad 10,48$

7,78

8,38

6,17

7,05

12,96 
TABELA 1 - Continuação.

PERIODOS

VACAS
1
2
4
5
6
7

97

$11,56 \quad 11,10$

11,76

10,92

10,16

9,57

9,11

98

$8,65 \quad 10,86$

11,65

11,64

9,56

9,03

8,98

99

$11,27 \quad 10,82$

10,75

10,95

9,93

8,51

6,61

100

11,06

11,92

11,82

10,85

10,26

9,35

7,08

101

8,65

8,13

8,04

6,56

8,97

8,03

7,05

102

$11,46 \quad 11,70$

11,58

14,23

12,40

10,76

10,17

103

$14,23 \quad 13,33$

13,59

12,34

13,09

12,22

9,59

104

$13,44 \quad 15,82$

13,23

15,10

15,17

15,35

16,10

105

11,62

11,74

12,31

10,22

10,68

11,12

8,78

106

10,89

8,92

7,86

8,25

7,38

7,32

7,14

107

9,94

8,70

9,33

10,91

10,11

9,36

6,36

108

9,12

8,58

8,34

8,08

7,96

7,23

7,20

109

9,64

11,40

10,90

8,07

8,59

7,72

6,84

110

$12,14 \quad 10,42$

9,54

9,11

6,82

6,28

5,68

111

8,67

8,76

7,97

7,88

8,11

7,46

7,32

112

7,56

5,78

4,76

7,60

11,02

10,41

6,15

113

11,67

10,11

10,57

10,92

9,31

8,67

8,12

114

8,47

7,93

7,32

7,37

6,67

5,22

4,67

115

13,31

13,44

12,41

12,65

11,35

9,49

9,06

116

11,34

8,48

$10,31 \quad 10,66$

9,26

8,24

8,12

117

12,34

11,45

$13,64 \quad 13,21$

13,56

12,11

12,17

118

11,45

14,95

14,54

13,74

13,19

13,65

11,59

119

9,71

10,03

8,52

8,58

7,53

7,90

8,10

120

7,65 .

8,72

8,38

8,60

8,18

7,74

7,69 
TABELA 1 - Continuação.

\begin{tabular}{|c|c|c|c|c|c|c|c|}
\hline \multirow{2}{*}{ VACAS } & \multicolumn{7}{|c|}{ PERIODOS } \\
\hline & 1 & 2 & 3 & 4 & 5 & 6 & 7 \\
\hline 121 & 9,90 & 9,22 & 9,15 & 9,06 & 9,26 & 8,74 & 9,76 \\
\hline 122 & 10,90 & 10,74 & 10,97 & 10,23 & 9,26 & 7,59 & 7,06 \\
\hline 123 & 12,85 & 10,05 & 7,47 & 7,88 & 8,66 & 7,69 & 6,58 \\
\hline 124 & 9,24 & 11,77 & 8,70 & 7,41 & 7,78 & 7,83 & 6,25 \\
\hline 125 & 7,54 & 8,85 & 8,28 & 8,40 & 7,90 & 6,76 & 6,32 \\
\hline 126 & 10,29 & 10,07 & 9,20 & 9,34 & 9,11 & 8,43 & 7,80 \\
\hline 127 & 14,40 & 12,33 & 14,34 & 13,39 & 12,06 & 10,96 & 10,53 \\
\hline 128 & 19,71 & 17,98 & 16,48 & 15,75 & 15,85 & 12,59 & 11,60 \\
\hline 129 & 9,72 & 9,94 & 8,84 & 8,21 & 7,66 & 7,73 & 5,17 \\
\hline 130 & 8,91 & 8,49 & 7,96 & 7,54 & 7,53 & 7,10 & 6,39 \\
\hline 131 & 12,07 & 12,17 & 10,94 & 9,09 . & 8,15 & 7,93 & 6,16 \\
\hline 132 & 12,40 & 14,45 & 15,09 & 16,82 & 11,44 & 10,84 & 9,45 \\
\hline 133 & 5,58 & 6,03 & 5,99 & 5,68 & 5,09 & 5,02 & 5,32 \\
\hline 134 & 8,68 & 9,06 & 8,32 & 8,27 & 7,50 & 6,91 & 6,05 \\
\hline 135 & 14,99 & 14,41 & 14,11 & 11,97 & 10,76 & 9,59 & 7,79 \\
\hline 136 & 8,81 & 8,08 & 8,10 & 7,71 & 7,51 & 7,39 & 6,62 \\
\hline 137 & 6,73 & 7,51 & 7,19 & 6,43 & 5,57 & 5,89 & 5,81 \\
\hline 138 & 8,96 & 8,61 & 7,93 & 7,86 & 6,84 & 6,82 & 5,73 \\
\hline 139 & 10,78 & 9,10 & 8,52 & 6,74 & 6,22 & 5,38 & 3,58 \\
\hline 140 & 7,62 & 6,81 & 7,45 & 8,01 & 7,52 & 4,88 & 5,83 \\
\hline 141 & 7,90 & 8,59 & 8,89 & 8,34 & 8,53 & 7,58 & 6,46 \\
\hline 142 & 9,23 & 8,51 & 8,36 & 5,95 & 6,63 & 6,25 & 4,80 \\
\hline 143 & 12,45 & 12,52 & 10,90 & 10,23 & 8,19 & 7,49 & 8,53 \\
\hline 144 & 15,30 & 14,96 & 12,84 & 12,33 & 14,14 & 14,19 & 11,34 \\
\hline
\end{tabular}


TABELA 2 - Correlações entre períodos estimados a partir de 144 lactações.

\begin{tabular}{|c|c|c|c|c|c|c|c|}
\hline \multirow{2}{*}{ PERIODOS } & \multicolumn{7}{|c|}{ PERIODOS } \\
\hline & 1 & 2 & 3 & 4 & 5 & 6 & 7 \\
\hline 1 & 1,000 & 0,897 & 0,846 & 0,773 & 0,750 & 0,676 & 0,642 \\
\hline 2 & - & 1,000 & 0,913 & 0,827 & 0,776 & 0,721 & 0,669 \\
\hline 3 & - & - & 1,000 & 0,909 & 0,818 & 0,762 & 0,712 \\
\hline 4 & - & - & - & 1,000 & 0,884 & 0,816 & 0,787 \\
\hline 5 & - & - & - & - & 1,000 & 0,930 & 0,845 \\
\hline 6 & - & - & - & - & - & 1,000 & 0,900 \\
\hline 7 & - & - & - & - & - & - & 1,000 \\
\hline
\end{tabular}


TABELA 3 - Correlações entre periodos a partir do modelo autoregressivo $(\rho=0,91)$.

\begin{tabular}{|c|c|c|c|c|c|c|c|}
\hline \multirow{2}{*}{ PERIODOS } & \multicolumn{7}{|c|}{ PERIODOS } \\
\hline & 1 & 2 & 3 & 4 & 5 & 6 & 7 \\
\hline 1 & 1,000 & 0,910 & 0,828 & 0,754 & 0,687 & 0,625 & 0,568 \\
\hline 2 & - & 1,000 & 0,910 & 0,828 & 0,754 & 0,687 & 0,625 \\
\hline 3 & - & - & 1,000 & 0,910 & 0,828 & 0,754 & 0,687 \\
\hline 4 & - & - & - & 1,000 & 0,910 & 0,828 & 0,754 \\
\hline 5 & - & - & - & - & 1,000 & 0,910 & 0,828 \\
\hline 6 & - & - & - & - & - & 1,000 & 0,910 \\
\hline 7 & - & - & - & - & - & - & 1,000 \\
\hline
\end{tabular}




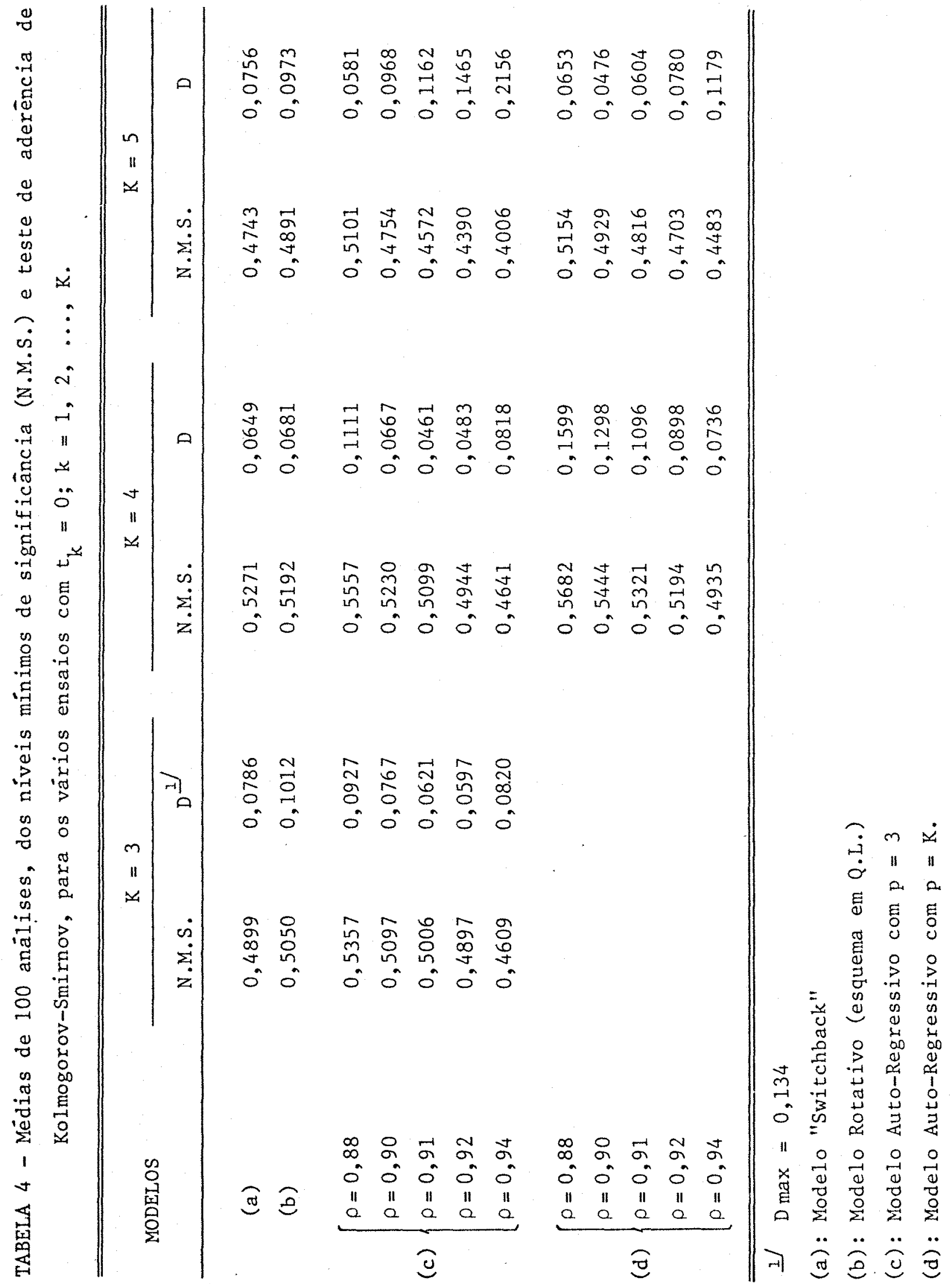




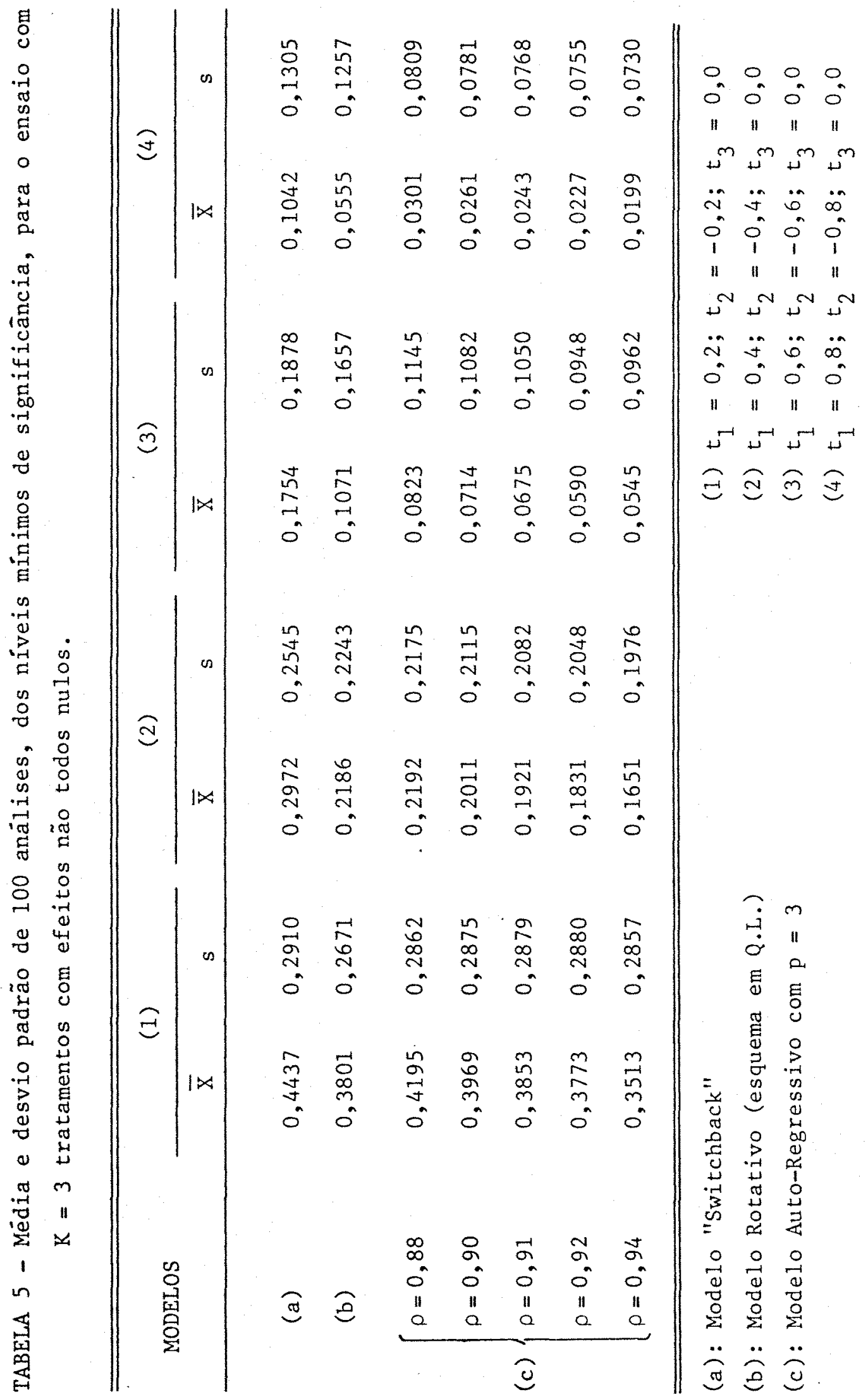




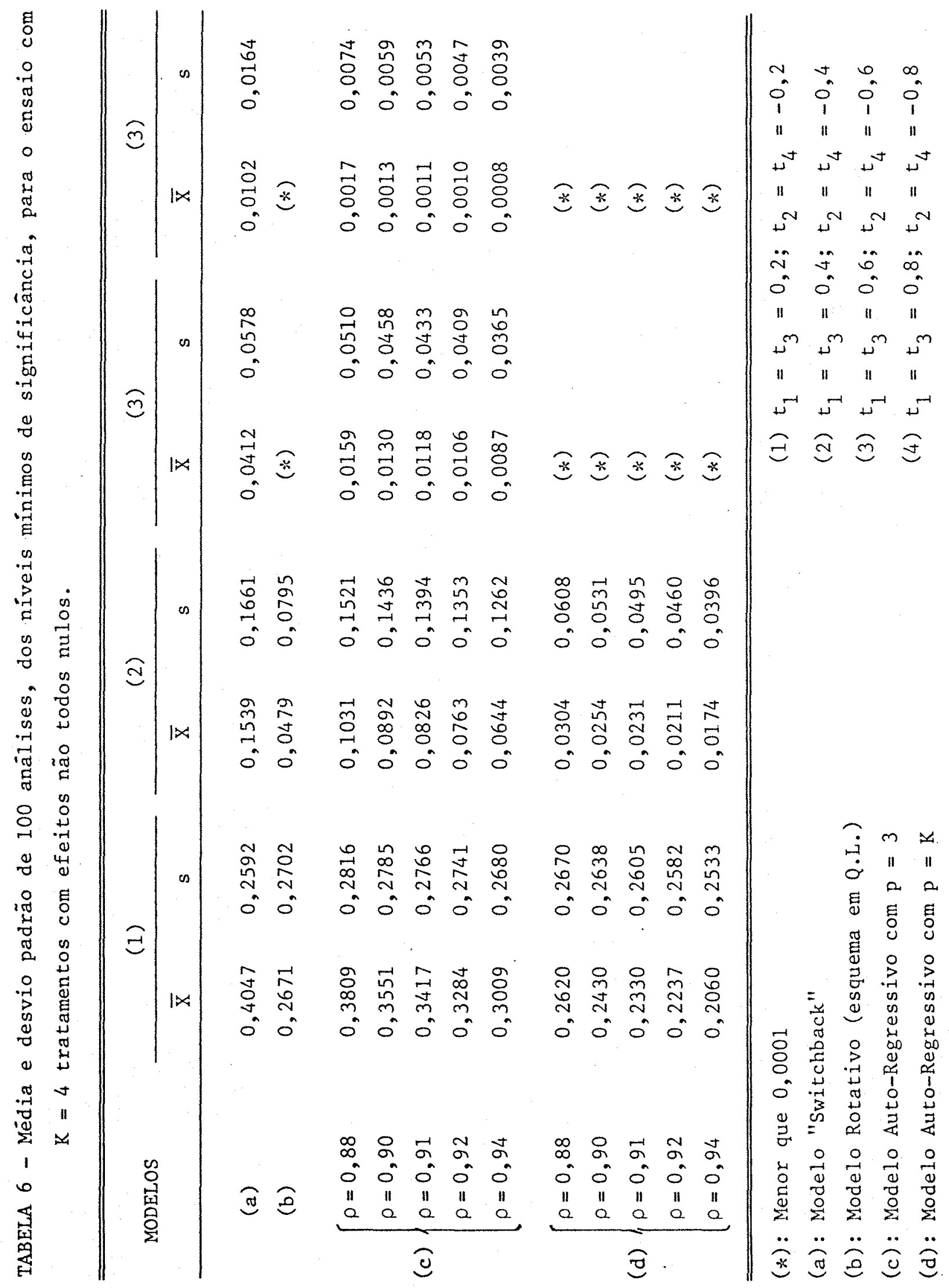




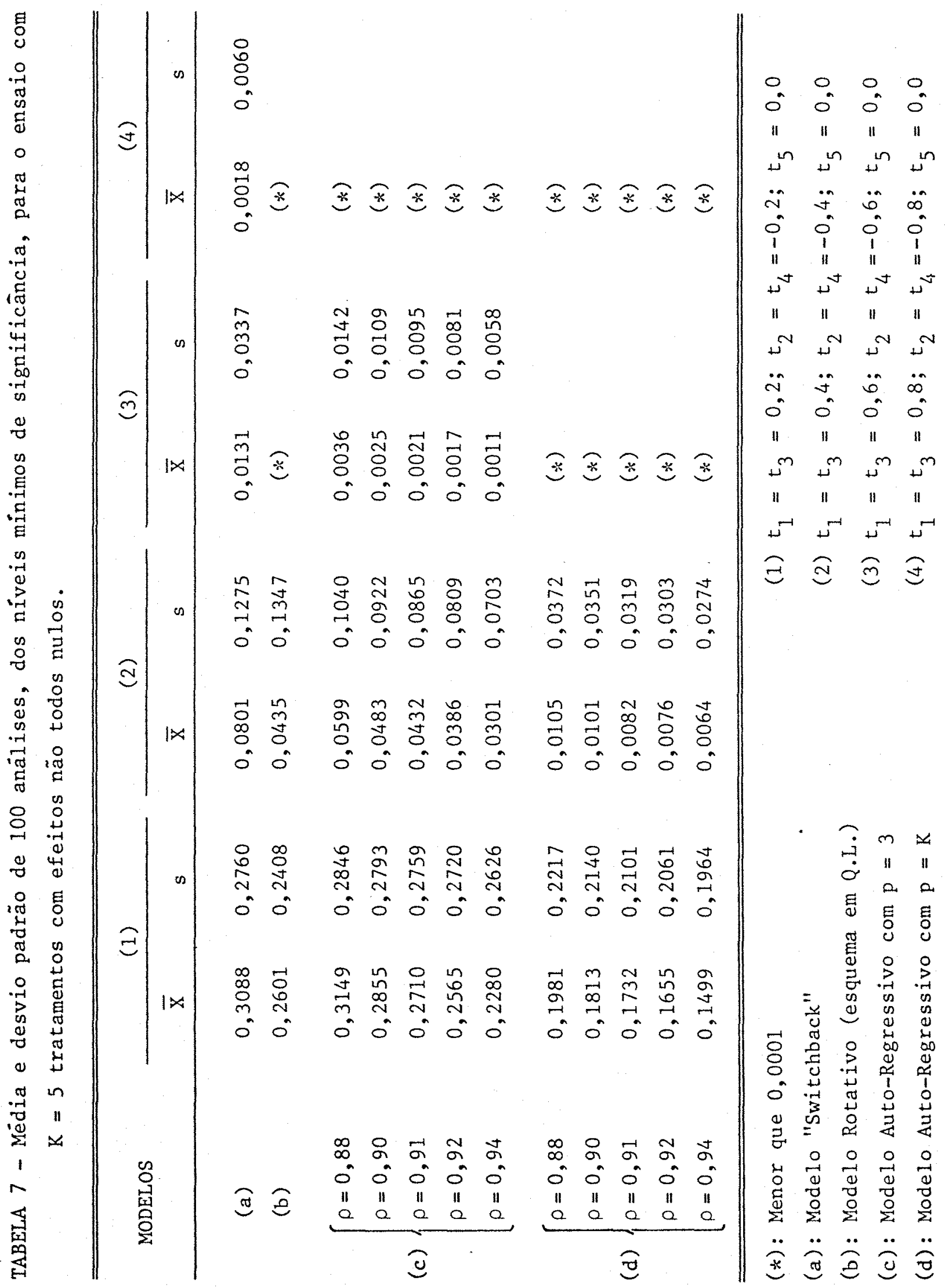


TABELA 8 - Distribuições de freqüencias para as 4 classes iniciais, dos ni veis minimos de significância, para o ensaio com $\mathrm{K}=3$ tratamen tos com efeitos não todos nulos.

\begin{tabular}{|c|c|c|c|c|c|c|c|c|}
\hline & \multirow{2}{*}{ CLASSES } & \multirow{2}{*}{ (a) } & \multirow{2}{*}{ (b) } & \multicolumn{5}{|c|}{ (c) } \\
\hline & & & & $\rho=0,88$ & $\rho=0,90$ & $\rho=0,91$ & $\rho=0,92$ & $\rho=0,94$ \\
\hline \multirow{4}{*}{ (1) } & {$[0,00 ; 0,05)$} & 6 & 10 & 6 & 7 & 10 & 13 & 14 \\
\hline & {$[0,05 ; 0,10)$} & 6 & 8 & 9 & 12 & 10 & 9 & 8 \\
\hline & {$[0,10 ; 0,15)$} & 8 & 7 & 7 & 5 & 6 & 5 & 6 \\
\hline & {$[0,15 ; 0,20)$} & 5 & 5 & 5 & 7. & 9 & 11 & 16 \\
\hline \multirow{4}{*}{ (2) } & {$[0,00 ; 0,05)$} & 16 & 28 & 29 & 33 & 36 & 39 & 41 \\
\hline & {$[0,05 ; 0,10)$} & 10 & 12 & 15 & 13 & 10 & 9 & 12 \\
\hline & {$[0,10 ; 0,15)$} & 13 & 12 & 7 & 9 & 11 & 10 & 12 \\
\hline & {$[0,15 ; 0,20)$} & 8 & 8 & 9 & 6 & 10 & 9 & 7 \\
\hline \multirow{4}{*}{ (3) } & {$[0,00 ; 0,05)$} & 28 & 53 & 58 & 62 & 67 & 70 & 79 \\
\hline & {$[0,05 ; 0,10)$} & 19 & 15 & 17 & 18 & 15 & 13 & 3 \\
\hline & {$[0,10 ; 0,15)$} & 9 & 12 & 6 & 4 & 2 & 3 & 5 \\
\hline & {$[0,15 ; 0,20)$} & 9 & 6 & 3 & 5 & 7 & 7 & 5 \\
\hline \multirow{4}{*}{ (4) } & {$[0,00 ; 0,05)$} & 46 & 80 & 87 & 90 & 91 & 92 & 92 \\
\hline & {$[0,05 ; 0,10)$} & 17 & 7 & 7 & 5 & 4 & 3 & 3 \\
\hline & {$[0,10 ; 0,15)$} & 17 & 6 & 2 & 1 & 1 & 2 & 2 \\
\hline & {$[0,15 ; 0,20)$} & 6 & 2 & 1 & 2 & 2 & 1 & 1 \\
\hline
\end{tabular}
(a): Modelo "Switchback"
(b) : Modelo Rotativo (esquema em Q.L.)
(c): Modelo Auto-Regressivo com $\mathrm{p}=3$
(1) $t_{1}=0,2 ; t_{2}=-0,2 ; t_{3}=0,0$
(2) $t_{1}=0,4 ; t_{2}=-0,4 ; t_{3}=0,0$
(3) $t_{1}=0,6 ; t_{2}=-0,6 ; t_{3}=0,0$
(4) $t_{1}=0,8 ; t_{2}=-0,8 ; t_{3}=0,0$ 


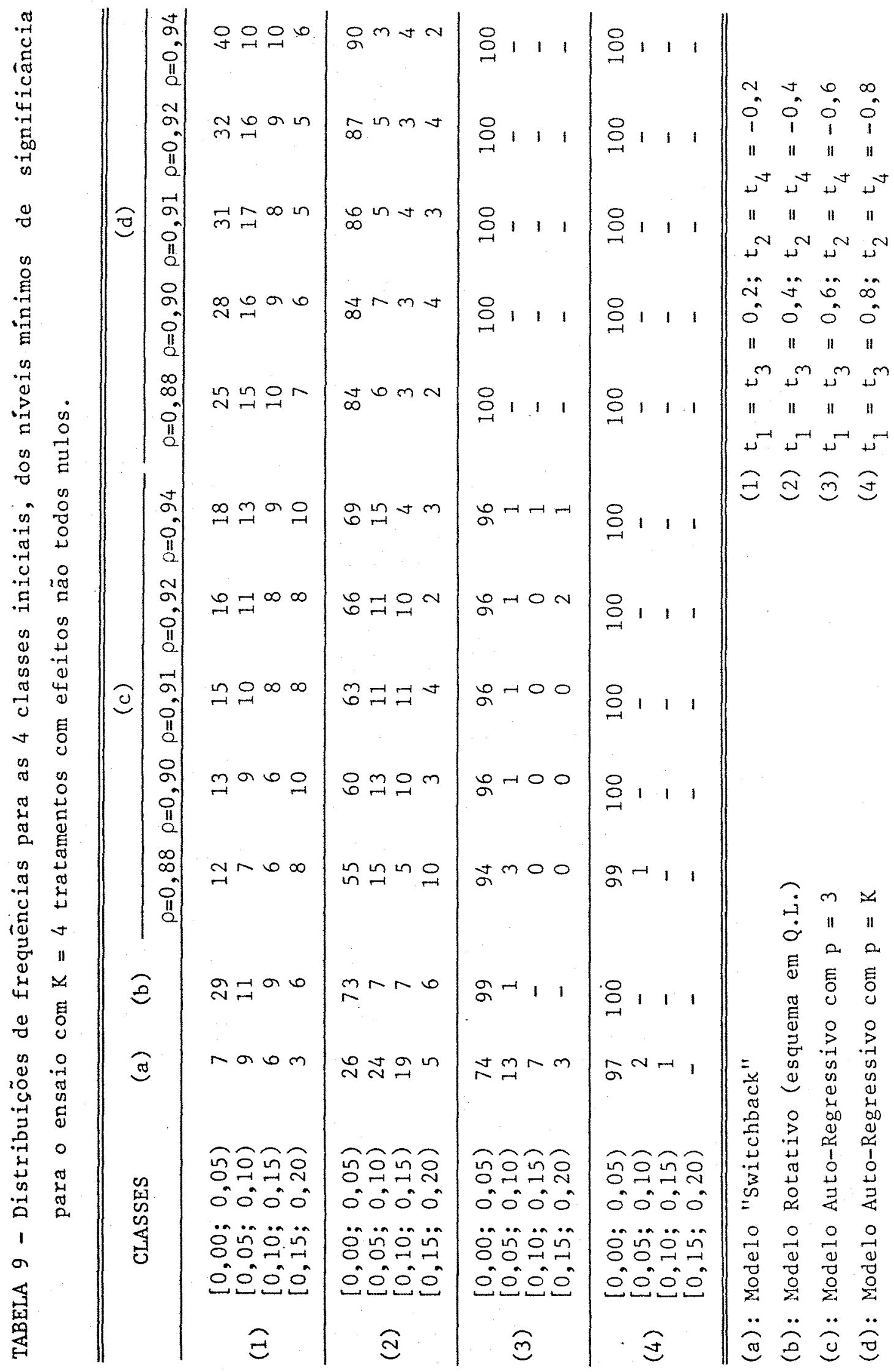




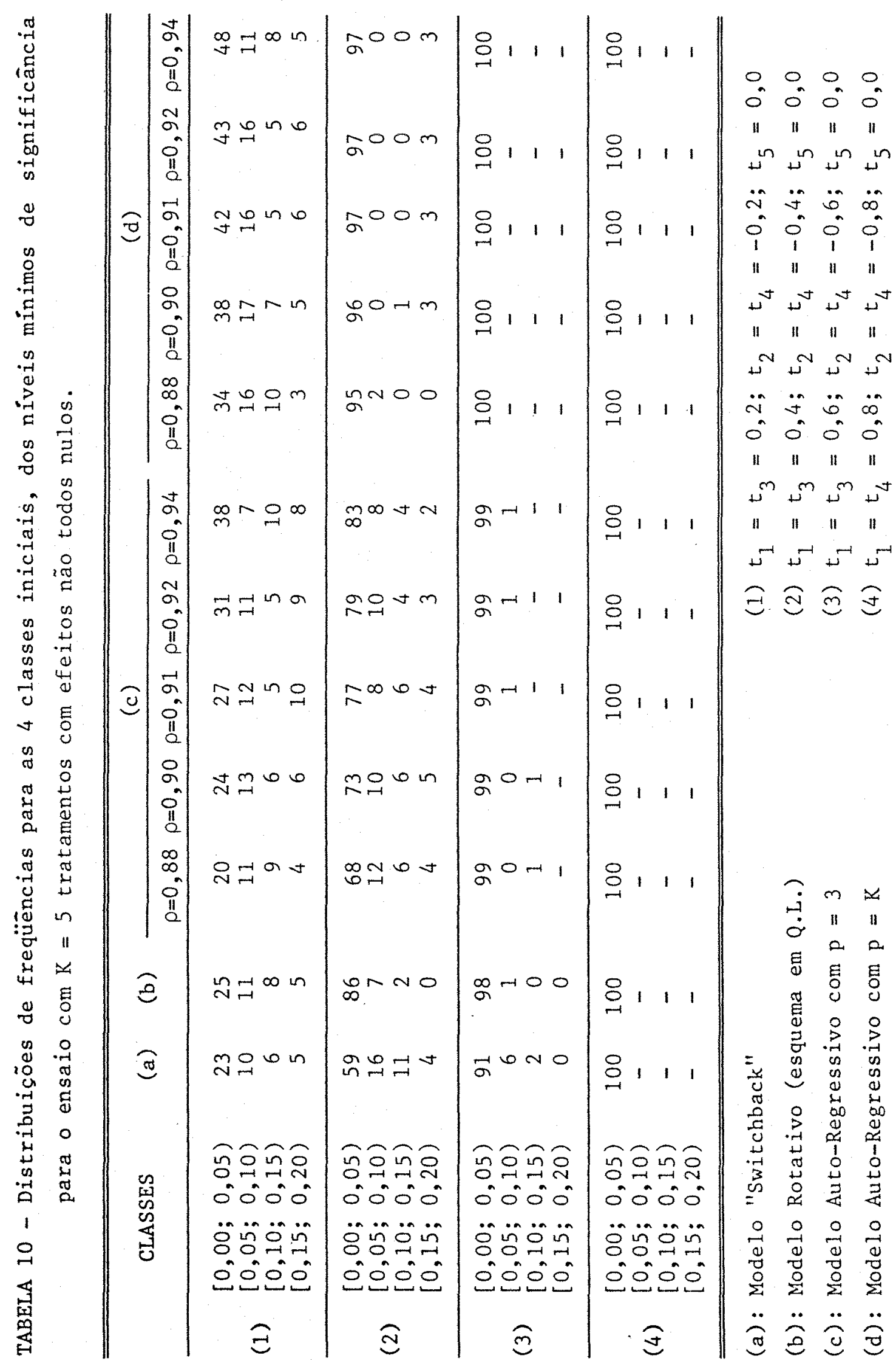




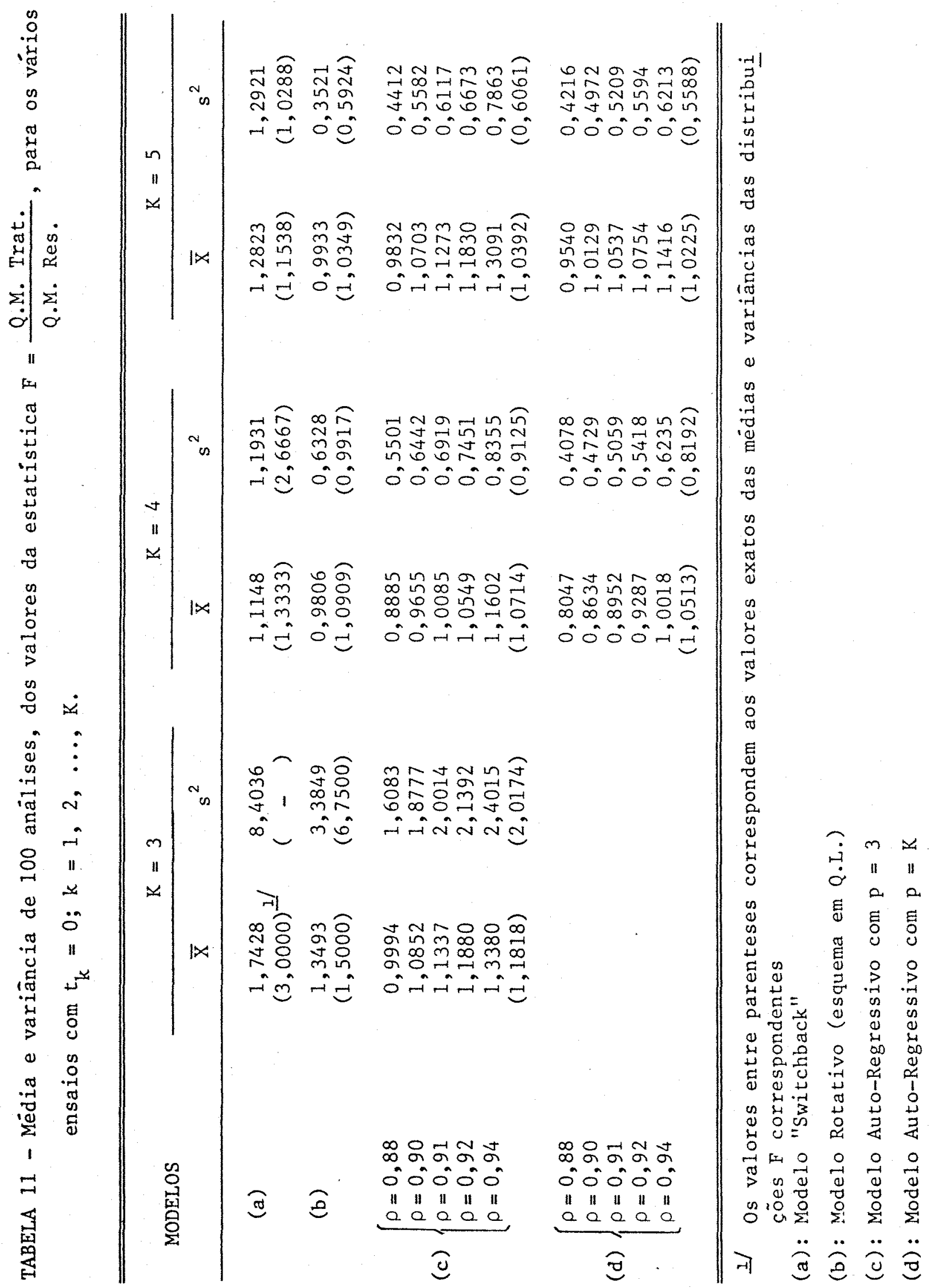


TABELA 12 - Quociente de médias de 100 anälises do Q.M. Trat. (aj.) pelo Q.M. Res., para o ensaio com $K=3$ tratamentos com efeitos não todos nulos.

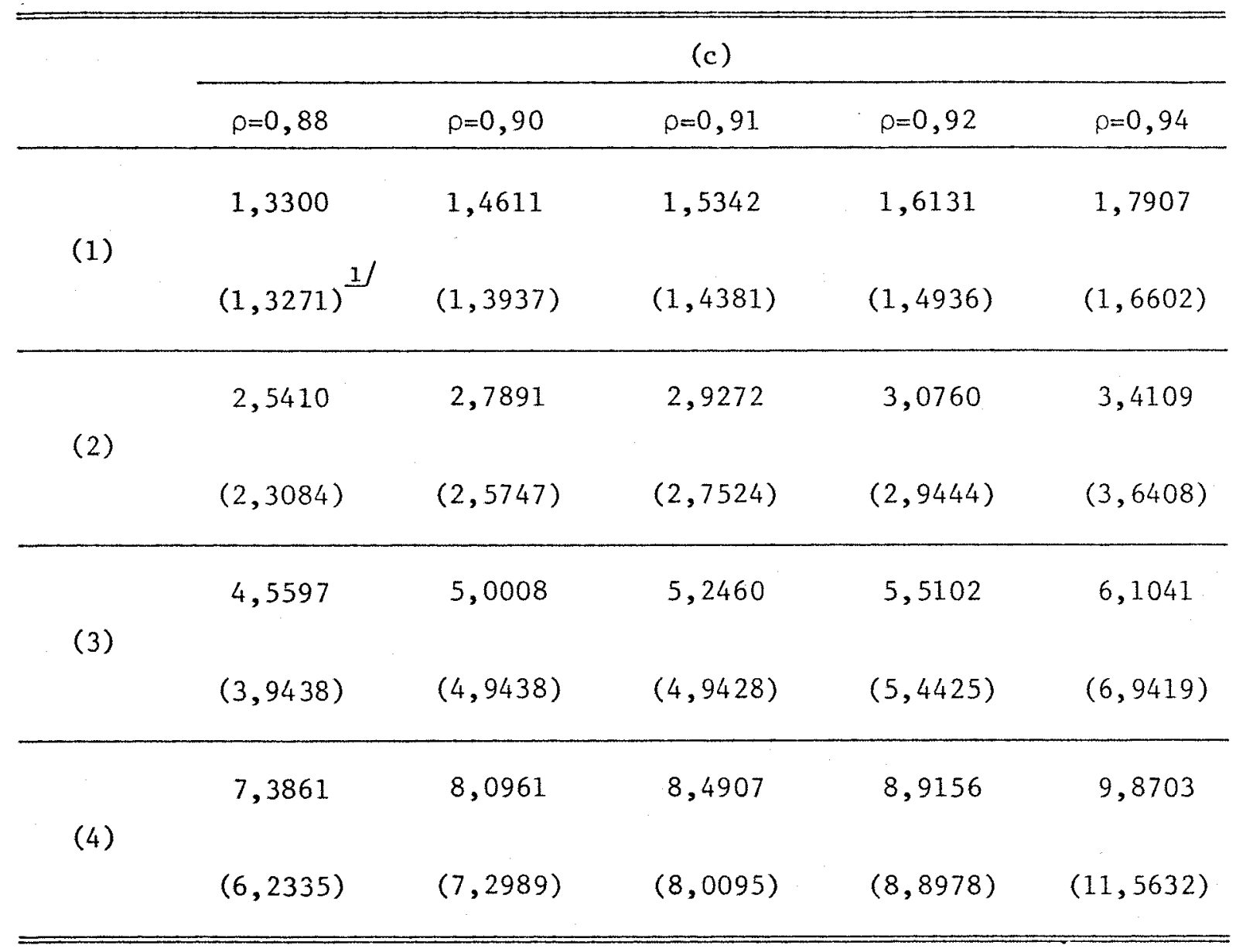

If Valores esperados segundo a expressão (66)

(c) Modelo Auto-Regressivo com $p=3$

(1) $t_{1}=0,2 ; t_{2}=-0,2 ; t_{3}=0$

(2) $t_{1}=0,4 ; t_{2}=-0,4 ; t_{3}=0$

(3) $t_{1}=0,6 ; t_{2}=-0,6 ; t_{3}=0$

(4) $t_{1}=0,8 ; t_{2}=-0,8 ; t_{3}=0$ 


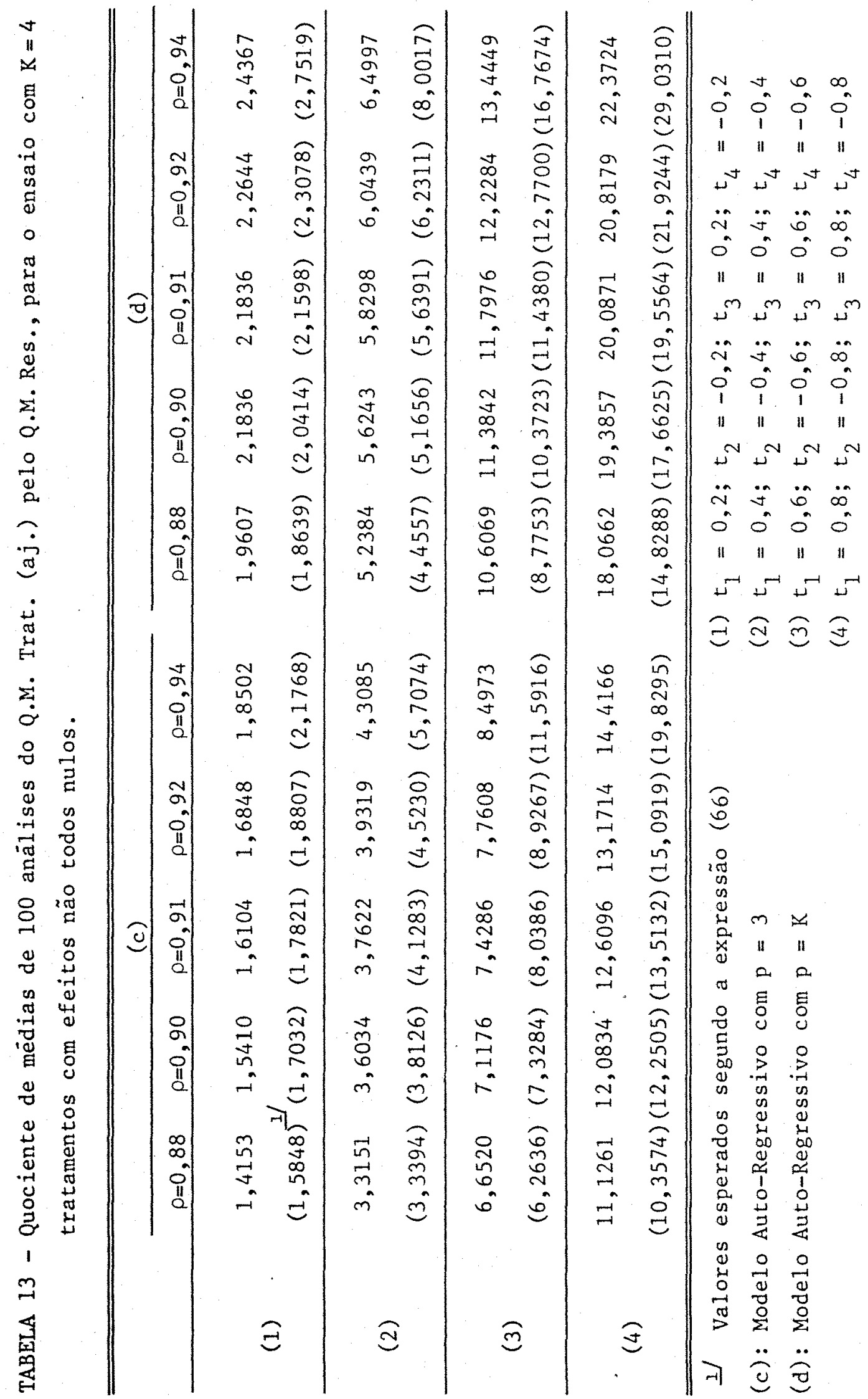




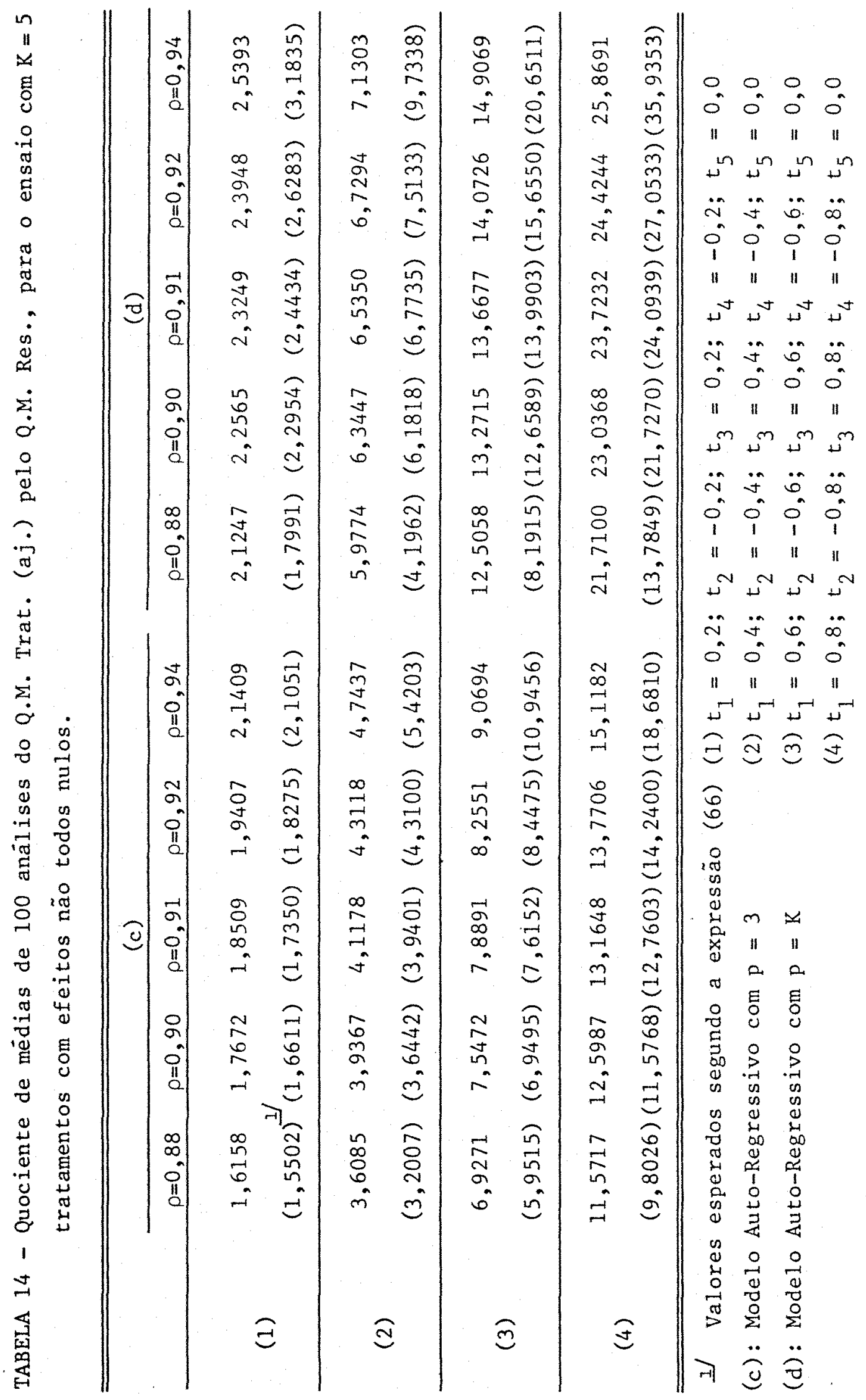




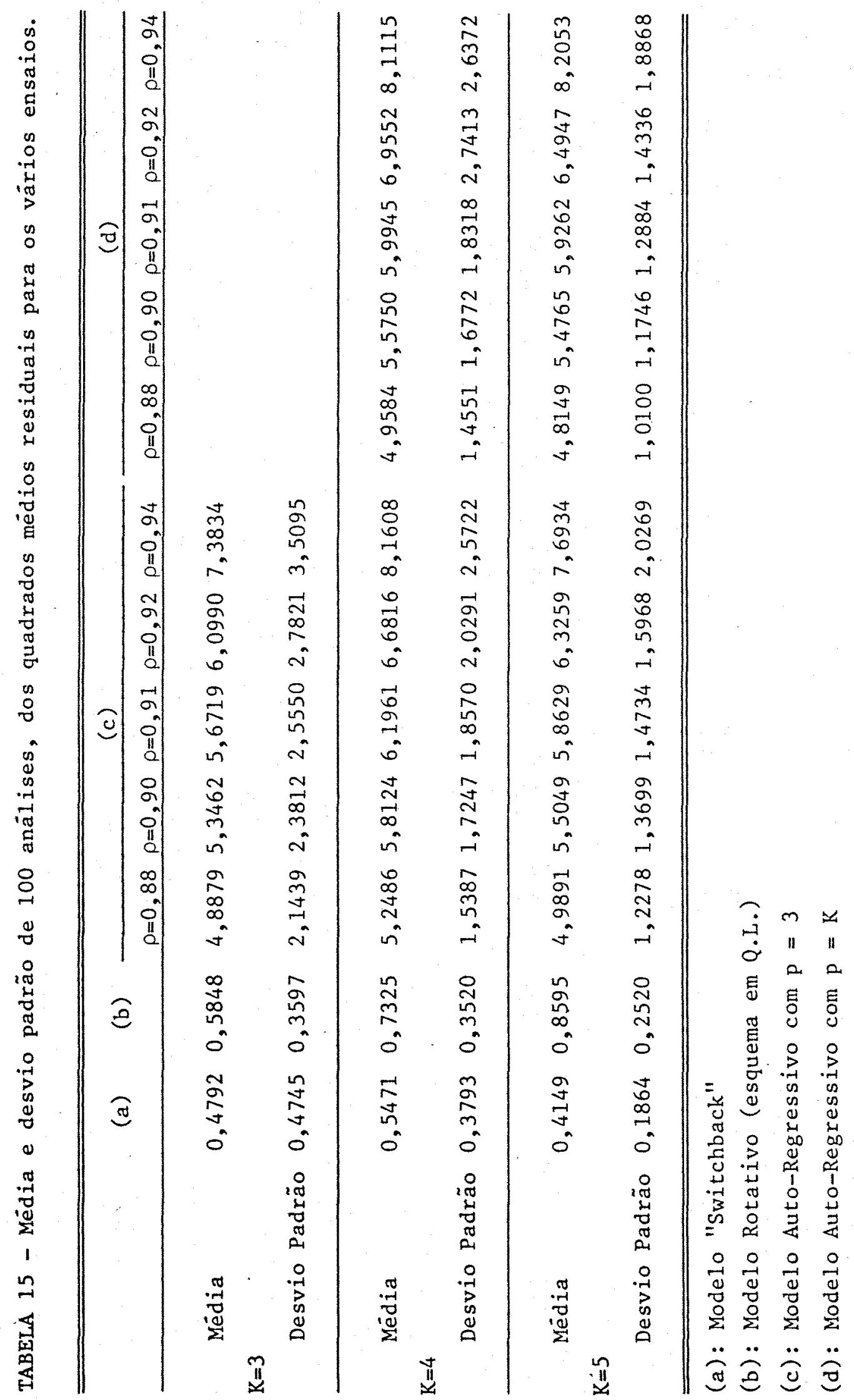

